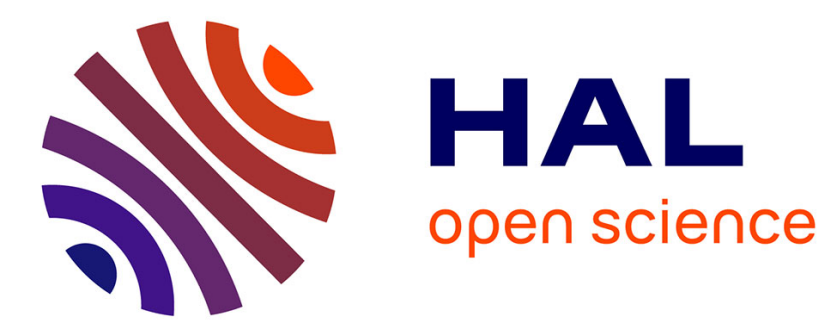

\title{
Non-Gaussian positive-definite matrix-valued random fields for elliptic stochastic partial differential operators
}

\author{
Christian Soize
}

\section{To cite this version:}

Christian Soize. Non-Gaussian positive-definite matrix-valued random fields for elliptic stochastic partial differential operators. Computer Methods in Applied Mechanics and Engineering, 2006, 195 (1-3), pp.26-64. 10.1016/j.cma.2004.12.014 . hal-00686157

\section{HAL Id: hal-00686157 https://hal.science/hal-00686157}

Submitted on 7 Apr 2012

HAL is a multi-disciplinary open access archive for the deposit and dissemination of scientific research documents, whether they are published or not. The documents may come from teaching and research institutions in France or abroad, or from public or private research centers.
L'archive ouverte pluridisciplinaire HAL, est destinée au dépôt et à la diffusion de documents scientifiques de niveau recherche, publiés ou non, émanant des établissements d'enseignement et de recherche français ou étrangers, des laboratoires publics ou privés. 


\title{
Non Gaussian positive-definite matrix-valued random fields for elliptic stochastic partial differential operators
}

\author{
C. Soize \\ Laboratory of Engineering Mechanics, University of Marne-la-Vallée, \\ 5 boulevard Descartes, 77454 Marne-la-Vallée, France, e-mail: soize@univ-mlv.fr
}

\begin{abstract}
This paper deals with the construction of a class of non Gaussian positive-definite matrix-valued random fields whose mathematical properties allow elliptic stochastic partial differential operators to be modeled. The properties of this class is studied in details and the numerical procedure for constructing numerical realizations of the trajectories is explicitly given. Such a matrix-valued random field can directly be used for modeling random uncertainties in computational sciences with a stochastic model having a small number of parameters. The class of random fields which can be approximated is presented and their experimental identification is analyzed. An example is given in three-dimensional linear elasticity for which the fourth-order elasticity tensor-valued random field is constructed for a random non homogeneous anisotropic elastic material.
\end{abstract}

Keywords: Random field; Non Gaussian; Uncertainties; Stochastic partial differential equations.

\section{Introduction}

The role played by the modeling of random uncertainties in computational sciences is become really important for improving the predictability of the numerical simulations. The fundamental mathematical tool used for performing such models is the probability theory which is very developed and allows numerous problems to be studied in sciences: see for instance $[9,15,28,41,42,60]$ for the theory of stochastic processes and random fields, $[28,29,32,33,40,51,57,60,70,86]$ for the theory of stochastic integration, stochastic differential equations and diffusion processes, $[52,55,56,72]$ for random signal processing, $[4,17,35,38,64]$ for mathematical statistics. These mathematical 
tools have been intensively used in the context of physics and engineering $[47,71,83]$, in particular for random vibration, for probabilistic theory and stochastic methods in structural dynamics and in structural acoustics, and for computational stochastic mechanics $[31,41,45,50,59,62,63,73]$.

In computational sciences, a predictive model is generally constructed by developing a mathematicalphysical model of a designed system in order to predict the response of the real system which is the system realized from the designed system. The mathematical-physical modeling process of the designed system introduces two fundamental types of uncertainties: the data uncertainties and the model uncertainties. Uncertainties have to be taken into account for improving the predictability of the model.

In general, model uncertainties cannot be modeled by using the usual parametric probabilistic approach. Recently, a nonparametric probabilistic approach of model uncertainties has been proposed in the context of dynamical systems using the random matrix theory and has been validated for different applications $[74-81,7,8,13]$. Such a nonparametric probabilistic approach is not directly addressed in the present paper. Nevertheless, the class of random fields which is constructed below corresponds to this nonparametric probabilistic approach.

Data uncertainties concern the parameters of the boundary value problem resulting from the mathematical-physical modeling of the designed system and are, for instance, the geometrical parameters, the boundary conditions, the coefficients of the partial differential operators of the problem, etc. The best approach to take into account data uncertainties is the parametric probabilistic approach which consists in modeling the parameters of the model by random quantities such as vector-valued random variables, vector-valued stochastic processes, tensor-valued random fields, etc. In the last period of fifteen years, a lot of works have been carried out in this area, in particular concerning the stochastic finite element method [14, 19 - 26, 39, 46, 69, 82, 84, 85, 87, 89] and other numerical methods for solving stochastic boundary value problems $[11,43,44]$. Such a parametric probabilistic approach is directly addressed in the present paper.

A great challenge is to construct stochastic representations of random parameters for which probabilistic data are known and can be identified by using experimental data. Let us consider, for instance, the following deterministic elliptic partial differential operator $\underline{A}$ on a bounded open domain $\Omega$ of $\mathbb{R}^{3}$, related to the three-dimensional linear elasticity for a non homogeneous anisotropic 
elastic material [10],

$$
\underline{A} \underline{\mathbf{v}}=-\sum_{i=1}^{3} \mathbf{e}^{i} \sum_{j=1}^{3} \frac{\partial}{\partial x_{j}}\left\{\sum_{k, h=1}^{3} \underline{c}_{i j k h}(\mathbf{x}) \varepsilon_{k h}(\underline{\mathbf{v}})\right\}
$$

in which $\mathbf{x}=\left(x_{1}, x_{2}, x_{3}\right) \in \Omega \subset \mathbb{R}^{3}$, where $\mathbf{e}^{1}=(1,0,0), \mathbf{e}^{2}=(0,1,0)$ and $\mathbf{e}^{3}=(0,0,1)$ are the vectors of the canonical basis of $\mathbb{R}^{3}$ and where $\mathbf{x} \mapsto \underline{\mathbf{v}}(\mathbf{x})=\left(\underline{v}_{1}(\mathbf{x}), \underline{v}_{2}(\mathbf{x}), \underline{v}_{3}(\mathbf{x})\right)$ is a twice differentiable function from $\Omega$ into $\mathbb{R}^{3}$. The second-order strain tensor $\left\{\varepsilon_{k h}\right\}_{k h}$ is such that

$$
\varepsilon_{k h}(\underline{\mathbf{v}})=\frac{1}{2}\left(\frac{\partial \underline{v}_{k}}{\partial x_{h}}+\frac{\partial \underline{v}_{h}}{\partial x_{k}}\right)
$$

The fourth-order real tensor $\underline{c}_{i j k h}(\mathbf{x})$ of the elastic coefficients verifies the usual property of symmetry

$$
\underline{c}_{i j k h}(\mathbf{x})=\underline{c}_{j i k h}(\mathbf{x})=\underline{c}_{i j h k}(\mathbf{x})=\underline{c}_{k h i j}(\mathbf{x})
$$

and for all symmetric second-order real tensors $\left\{z_{i j}\right\}_{i j}$, the tensor $\underline{c}_{i j k h}(\mathbf{x})$ verifies the following positive-definiteness property,

$$
\sum_{i, j, k, h=1}^{3} \underline{c}_{i j k h}(\mathbf{x}) z_{k h} z_{i j} \geq \underline{c}_{0} \sum_{i, j=1}^{3} z_{i j}^{2}
$$

in which $\underline{c}_{0}$ is a positive constant independent of $\mathbf{x}$. The fourth-order tensor $\underline{c}_{i j h k}(\mathbf{x})$ is constituted of 21 algebraically independent coefficients depending on $\mathbf{x}$. Let us assume that the material is random. Consequently, for all $\mathbf{x}$ fixed in $\Omega$, tensor $\left\{\underline{c}_{i j k h}(\mathbf{x})\right\}_{i j k h}$ is replaced by a fourth-order tensor-valued random variable $\left\{C_{i j k h}(\mathbf{x})\right\}_{i j k h}$ whose mean value is $\left\{\underline{c}_{i j k h}(\mathbf{x})\right\}_{i j k h}$ and which has to verify similar equations to Eqs. (2) and (3) in a probabilistic sense which has to be defined. Nevertheless, for the random case, the introduction of a deterministic constant $\underline{c}_{0}$ in Eq. (3) cannot always be justified from a probabilistic modeling point of view (this statement will be explained in Section 5.6). Finally, $\mathbf{x} \mapsto\left\{C_{i j k h}(\mathbf{x})\right\}_{i j k h}$ is a fourth-order tensor-valued random field indexed by $\Omega$ and is constituted of 21 mutually dependent random fields. The stochastic partial differential operator $\mathbf{A}$ associated with operator $\underline{A}$ is such that

$$
\mathbf{A} \mathbf{V}=-\sum_{i}^{3} \mathbf{e}^{i} \sum_{j=1}^{3} \frac{\partial}{\partial x_{j}}\left\{\sum_{k, h=1}^{3} C_{i j k h}(\mathbf{x}) \varepsilon_{k h}(\mathbf{V})\right\}
$$

It should be noted that the probability distribution of this fourth-order tensor-valued random field (that is to say the system of the marginal probability distributions) is required because the unknown 
solution of the stochastic boundary value problem is a nonlinear mapping of the random field $\mathbf{x} \mapsto\left\{C_{i j k h}(\mathbf{x})\right\}_{i j k h}$. If the usual parametric probabilistic approach is used, then the identification of this probability model by using experimental data seems to be difficult.

This paper deals with a nonparametric construction of a random field such as $\mathbf{x} \mapsto\left\{C_{i j k h}(\mathbf{x})\right\}_{i j k h}$. For that, an ensemble of non Gaussian positive-definite matrix-valued random fields is constructed and studied which allows the fourth-order tensor-valued random field $\mathbf{x} \mapsto\left\{C_{i j k h}(\mathbf{x})\right\}_{i j k h}$ to be modeled. Then, such a tensor-valued random field will depend only on 4 scalar parameters: three spatial correlation lengths and one parameter allowing the level of the random fluctuations to be controlled. With such a model, the inverse problem related to the experimental identification seems to be more feasible.

It should be noted that the probabilistic model of a random field such as $\mathbf{x} \mapsto\left\{C_{i j k h}(\mathbf{x})\right\}_{i j k h}$ is constructed by using the tensor-valued field $\mathbf{x} \mapsto\left\{\underline{c}_{i j k h}(\mathbf{x})\right\}_{i j k h}$ of the associated mean model which is such that the mathematical expectation of the random tensor $\left\{C_{i j k h}(\mathbf{x})\right\}_{i j k h}$ is the deterministic tensor $\left\{\underline{c}_{i j k h}(\mathbf{x})\right\}_{i j k h}$. For instance, for heterogeneous elastic materials, the known symmetries, such as monoclinic symmetry, orthotropic symmetry, transverse square symmetry, transversaly isotropic symmetry, isotropic symmetry, can be taken into account with the mean model represented by the tensor $\left\{\underline{c}_{i j k h}(\mathbf{x})\right\}_{i j k h}$. Nevertheless, this paper is limited to the case for which the random tensor fluctuation $\left\{C_{i j k h}(\mathbf{x})-\underline{c}_{i j k h}(\mathbf{x})\right\}_{i j k h}$ around the mean model is purely anisotropic, without any symmetries.

Section 3 deals with the construction and the properties of the ensemble $\mathrm{SFG}^{+}$of normalized homogeneous non Gaussian positive-definite matrix-valued random fields. This ensemble constitutes the germs of the random fields in the ensemble $\mathrm{SFE}^{+}$which is defined and studied in Section 4. This ensemble is constituted of non normalized, non homogeneous and non Gaussian positive-definite matrix-valued random fields. In Section 5, we present the nonparametric probability modeling of elliptic stochastic partial differential operators on a bounded domain of $\mathbb{R}^{d}$, using the random fields belonging to $\mathrm{SFE}^{+}$. Section 6 is devoted to finite element discretization of such an elliptic stochastic partial differential operator on a bounded domain. Section 7 deals with the class of random fields which can be approximated with the ensemble $\mathrm{SFE}^{+}$and their experimental identification. Finally, a numerical example is presented in Section 8.

\section{Algebraic notations}

C. Soize - CMAME - revised version December 2004 
In this paper, the following algebraic notations are used.

Euclidean space. Let $\mathbf{x}=\left(x_{1}, \ldots, x_{n}\right)$ be a vector in $\mathbb{R}^{n}$. The Euclidean space $\mathbb{R}^{n}$ is equipped with the usual inner product $(\mathbf{x}, \mathbf{y}) \mapsto<\mathbf{x}, \mathbf{y}>=\sum_{j=1}^{n} x_{j} y_{j}$ and the associated norm $\|\mathbf{x}\|=<\mathbf{x}, \mathbf{x}>^{1 / 2}$. Matrix sets. Let $\mathbb{M}_{n, m}(\mathbb{R})$ be the set of all the $(n \times m)$ real matrices, $M_{n}(\mathbb{R})=M_{n, n}(\mathbb{R})$ be the set of all the square $(n \times n)$ real matrices, $\mathbb{M}_{n}^{S}(\mathbb{R})$ be the set of all the $(n \times n)$ real symmetric matrices and $\mathbb{M}_{n}^{+}(\mathbb{R})$ be the set of all the $(n \times n)$ real symmetric positive-definite matrices. We then have

$$
\mathbb{M}_{n}^{+}(\mathbb{R}) \subset \mathbb{M}_{n}^{S}(\mathbb{R}) \subset \mathbb{M}_{n}(\mathbb{R})
$$

Norms and usual operators. We denote:

(i) The determinant of matrix $[A] \in \mathbb{M}_{n}(\mathbb{R})$ as $\operatorname{det}[A]$ and its trace $\operatorname{as} \operatorname{tr}[A]=\sum_{j=1}^{n}[A]_{j j}$.

(ii) The transpose of $[A] \in \mathbb{M}_{n, m}(\mathbb{R})$ as $[A]^{T} \in \mathbb{M}_{m, n}(\mathbb{R})$.

(iii) The operator norm of the matrix $[A] \in \mathbb{M}_{n, m}(\mathbb{R})$ as

$$
\|A\|=\sup _{\|\mathbf{x}\| \leq 1}\|[A] \mathbf{x}\| \quad, \quad \mathbf{x} \in \mathbb{R}^{m},
$$

which is such that $\|[A] \mathbf{x}\| \leq\|A\|\|\mathbf{x}\|, \forall \mathbf{x} \in \mathbb{R}^{m}$. If $m=n$, then $\|A\|=\left|\lambda_{n}\right|$ in which $\left|\lambda_{n}\right|$ is the largest modulus of the eigenvalues of $[A]$.

(iv) For $[A]$ and $[B] \in \mathbb{M}_{n, m}(\mathbb{R})$, we denote $\ll[A],[B] \gg=\operatorname{tr}\left\{[A]^{T}[B]\right\}$ and the Frobenius norm (or Hilbert-Schmidt norm) $\|A\|_{F}$ of $[A]$ is such that

$$
\|A\|_{F}^{2}=\ll[A],[A] \gg=\operatorname{tr}\left\{[A]^{T}[A]\right\}=\sum_{j=1}^{n} \sum_{k=1}^{m}[A]_{j k}^{2}
$$

For $[A]$ in $M_{n}(\mathbb{R})$, we have

$$
\|A\| \leq\|A\|_{F} \leq \sqrt{n}\|A\|
$$

\section{Construction and properties of the ensemble $\mathrm{SFG}^{+}$of homogeneous and normalized non}

\section{Gaussian positive-definite matrix-valued random fields}

\subsection{Principle of the construction}

Let $n \geq 2$ and $d \geq 1$ be two finite integers. In Refs. [74,75,81], we introduced the ensemble $\mathrm{SG}^{+}$ of the random matrices $\left[\mathbf{G}_{n}\right]$, defined on a probability space $(\Theta, \mathcal{T}, P)$ with values in $\mathbb{M}_{n}^{+}(\mathbb{R})$, whose probability distribution was constructed by using the entropy optimization principle [65,34,36,37]

C. Soize - CMAME - revised version December 2004 
with the constraints defined by the following available information: $\left[\mathbf{G}_{n}\right]$ is a symmetric positivedefinite real random matrix whose mean value is the identity matrix and for which the second-order moment of the random variable $\left\|\left[\mathbf{G}_{n}\right]^{-1}\right\|_{F}$ is finite. The probability distribution $P_{\left[\mathbf{G}_{n}\right]}$ on $\mathbb{M}_{n}^{+}(\mathbb{R})$ of such a random matrix $\left[\mathbf{G}_{n}\right]$ was explicitly constructed and depends only on dimension $n$ and a positive real parameter $\delta$ independent of $n$ and allowing the dispersion of random matrix $\left[\mathbf{G}_{n}\right]$ to be controlled. Consequently, for $n$ fixed, $\mathrm{SG}^{+}$is a one-parameter ensemble of random matrices which differs from the usual ensemble of the random matrix theory [48]. In addition, it was proved that a random matrix belonging to $\mathrm{SG}^{+}$can be written as $\left[\mathbf{G}_{n}\right]=\left[\mathbf{L}_{n}\right]^{T}\left[\mathbf{L}_{n}\right]$ in which $\left[\mathbf{L}_{n}\right]$ is an upper triangular matrix with values in $\mathbb{M}_{n}(\mathbb{R})$ whose elements are constituted of $\nu=n(n+1) / 2$ independent random variables which can be written as a nonlinear transformation of $\nu$ independent copies of a real-valued Gaussian random variable $U$ with a zero mean and a variance equal to 1 .

The ensemble $\mathrm{SFG}^{+}$is then constructed as an ensemble of the random fields $\mathbf{x} \mapsto\left[\mathbf{G}_{n}(\mathbf{x})\right]$ defined on $(\Theta, \mathcal{T}, P)$ indexed by $\mathbb{R}^{d}$, with values in $\mathrm{SG}^{+}$, for which the $\nu$ independent real-valued Gaussian random variables are substituted by $\nu$ independent copies of a second-order homogeneous realvalued Gaussian random field $\mathbf{x} \mapsto U(\mathbf{x})$ indexed by $\mathbb{R}^{d}$, with $E\{U(\mathbf{x})\}=0, E\left\{U(\mathbf{x})^{2}\right\}=1(E$ is the mathematical expectation). The autocorrelation function $R_{U}(\boldsymbol{\eta})=E\{U(\mathbf{x}+\boldsymbol{\eta}) U(\mathbf{x})\}$ defined on $\mathbb{R}^{d}$ has to be constructed and will depend only on $d$ scalar parameters which will be the spatial correlation lengths associated with each coordinate $x_{1}, \ldots, x_{d}$. Clearly, for such a construction, any autocorrelation function $R_{U}(\boldsymbol{\eta})$ could be chosen. However, the aurocorrelation function $R_{U}(\boldsymbol{\eta})$ introduced in Section 3.2.2 has been constructed (1) to obtain the simplest model, (2) to get the required mathematical properties for random field $U$ and (3) to be completely defined with the smaller number of parameters (that is to say, by $d$ scalar parameters).

Consequently, for $n$ and $d$ fixed, ensemble $\mathrm{SFG}^{+}$will be a $(1+d)$-parameters ensemble of random fields. This ensemble is constituted of non Gaussian homogeneous and normalized random fields indexed by $\mathbb{R}^{d}$, with values in $\mathbb{M}_{n}^{+}(\mathbb{R})$, for which random field $U$ has to be considered as the "germ". We will use this ensemble for constructing the ensemble $\mathrm{SFE}^{+}$of non homogeneous (or homogeneous) and non normalized random fields indexed by any open set $\Omega$ in $\mathbb{R}^{d}$ and with values in $M_{n}^{+}(\mathbb{R})$.

\subsection{Set $\mathcal{E}_{U}$ for germ $U$ of ensemble $\mathrm{SFG}^{+}$}

In this section, $\mathcal{E}_{U}$ is defined as the set of all the random fields $U$ having given properties. A random field belonging to $\mathcal{E}_{U}$ will be the germ of the ensemble $\mathrm{SFG}^{+}$. An explicit construction of a random 
field $U$ in $\mathcal{E}_{U}$ is given.

\subsubsection{Definition of the set $\mathcal{E}_{U}$ of random fields $U$}

Let $d \geq 1$ be an integer. Let $U$ be any random field in the set $\mathcal{E}_{U}$. Then $\mathbf{x} \mapsto U(\mathbf{x})$ is a secondorder mean-square continuous homogeneous Gaussian random field defined on probability space $(\Theta, \mathcal{T}, P)$ indexed by $\mathbb{R}^{d}$, with values in $\mathbb{R}$, such that

$$
\forall \mathbf{x} \in \mathbb{R}^{d} \quad, \quad E\{U(\mathbf{x})\}=0 \quad, \quad E\left\{U(\mathbf{x})^{2}\right\}=1 .
$$

Its autocorrelation function $\boldsymbol{\eta}=\left(\eta_{1}, \ldots, \eta_{d}\right) \mapsto R_{U}(\boldsymbol{\eta})=E\{U(\mathbf{x}+\boldsymbol{\eta}) U(\mathbf{x})\}$ which is continuous from $\mathbb{R}^{d}$ into $\mathbb{R}$ is assumed to be such that

$$
R_{U}([C] \widetilde{\boldsymbol{\eta}})=1-\|\widetilde{\boldsymbol{\eta}}\|^{2}+o\left(\|\widetilde{\boldsymbol{\eta}}\|^{2}\right) \quad \text { for } \quad\|\widetilde{\boldsymbol{\eta}}\| \rightarrow 0
$$

in which $[C]$ is an invertible $(d \times d)$ real matrix and where the change of variable $\boldsymbol{\eta}=[C] \widetilde{\boldsymbol{\eta}}$ has been used.

From Eq. (6), it is deduced that, for $\|\boldsymbol{\eta}\| \rightarrow 0$,

$$
E\left\{\|U(\mathbf{x}+\boldsymbol{\eta})-U(\mathbf{x})\|^{2}\right\}=2\left(1-R_{U}(\boldsymbol{\eta})\right) \leq c\|\boldsymbol{\eta}\|^{2} \leq \frac{c}{|\log (\|\boldsymbol{\eta}\|)|^{1+\delta}}
$$

with $\delta>0$ and $c=2\left\|[C]^{-1}\right\|^{2}$. Using the Dudley lemma [16], it can be deduced that the random field $U$ has a continous version which means that the trajectories of the random field $U$ are continuous from $\mathbb{R}^{d}$ in $\mathbb{R}$ almost surely, that is to say, $\{\mathbf{x} \mapsto U(\mathbf{x})\} \in \mathcal{C}^{0}\left(\mathbb{R}^{d}, \mathbb{R}\right)$ almost surely. Finally, let $\mathbf{k}=\left(k_{1}, \ldots, k_{d}\right)$ be a point in $\mathbb{R}^{d}$ and let $d \mathbf{k}=d k_{1} \ldots d k_{d}$ be the Lebesgue measure. It is assumed that the power spectral density function $\mathbf{k} \mapsto S_{U}(\mathbf{k})$ from $\mathbb{R}^{d}$ into $\mathbb{R}^{+}$such that

$$
\forall \boldsymbol{\eta} \in \mathbb{R}^{d} \quad, \quad R_{U}(\boldsymbol{\eta})=\int_{\mathbb{R}^{d}} e^{i<\boldsymbol{\eta}, \mathbf{k}>} S_{U}(\mathbf{k}) d \mathbf{k}
$$

has a compact support which is written as

$$
\operatorname{supp} S_{U}=\left[-K_{1}, K_{1}\right] \times \ldots \times\left[-K_{d}, K_{d}\right],
$$

in which $K_{1}, \ldots, K_{d}$ are positive real numbers. Consequently, the mean-square partial derivative $\left\{D_{p_{1} \ldots p_{d}} U(\mathbf{x}), \mathbf{x} \in \mathbb{R}^{d}\right\}$ with

$$
D_{p_{1} \ldots p_{d}} U(\mathbf{x})=\frac{\partial^{p_{1}+\ldots+p_{d}}}{\partial x_{1}^{p_{1}} \ldots \partial x_{d}^{p_{d}}} U(\mathbf{x})
$$


is a mean-square continuous random field existing for all integers $p_{1} \geq 0, \ldots, p_{d} \geq 0$. The spatial correlation length $L_{j}^{U}$ of random field $U$ relative to coordinate $x_{j}$ is then defined

$$
L_{j}^{U}=\int_{0}^{+\infty}\left|R_{U}\left(0, \ldots, 0, \eta_{j}, 0, \ldots, 0\right)\right| d \eta_{j}
$$

\subsubsection{An example of random field $U$ in $\mathcal{E}_{U}$}

We consider a random field $\mathbf{x} \mapsto U(\mathbf{x})$ in $\mathcal{E}_{U}$ defined in Section 3.2.1, whose autocorrelation function is written as

$$
R_{U}(\boldsymbol{\eta})=\rho_{1}\left(\eta_{1}\right) \times \ldots \times \rho_{d}\left(\eta_{d}\right)
$$

in which, for all $j=1, \ldots, d$,

$$
\rho_{j}(0)=1 \quad ; \quad \rho_{j}\left(\eta_{j}\right)=\frac{4 L_{j}^{2}}{\pi^{2} \eta_{j}^{2}} \sin ^{2}\left(\frac{\pi \eta_{j}}{2 L_{j}}\right) \quad \text { for } \quad \eta_{j} \neq 0
$$

and where $L_{1}, \ldots, L_{d}$ are positive real numbers. Then, the power spectral density function $\mathbf{k}=$ $\left(k_{1}, \ldots, k_{d}\right) \mapsto S_{U}(\mathbf{k})$ from $\mathbb{R}^{d}$ into $\mathbb{R}^{+}$is written as

$$
S_{U}(\mathbf{k})=s_{1}\left(k_{1}\right) \times \ldots \times s_{d}\left(k_{d}\right)
$$

in which, for all $j=1, \ldots, d$, the function $k_{j} \mapsto s_{j}\left(k_{j}\right)$ from $\mathbb{R}$ into $\mathbb{R}^{+}$is defined by

$$
s_{j}\left(k_{j}\right)=\frac{L_{j}}{\pi} q\left(k_{j} L_{j} / \pi\right)
$$

In Eq. (13), the function $\tau \mapsto q(\tau)$ is continuous from $\mathbb{R}$ into $\mathbb{R}^{+}$, has a compact support $[-1,1]$ and is such that

$$
q(0)=1 \quad ; \quad q(-\tau)=q(\tau) \quad ; \quad q(\tau)=1-\tau \quad \text { for } \quad \tau \in[0,1]
$$

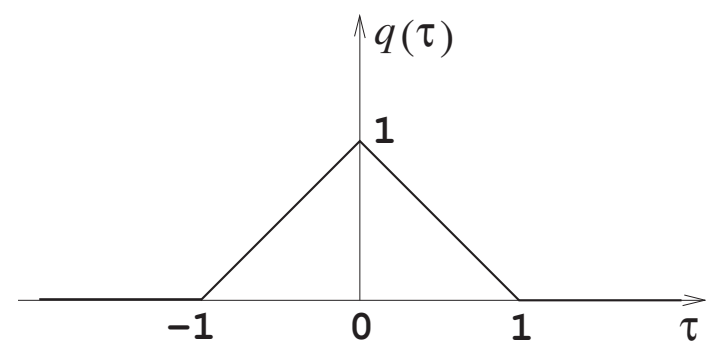

Fig. 1. Graph of function $\tau \mapsto q(\tau)$ 
Figure 1 displays the graph of function $\tau \mapsto q(\tau)$. Consequently, $S_{U}$ is a function with a compact support

$$
\operatorname{supp} S_{U}=\left[-K_{1}, K_{1}\right] \times \ldots \times\left[-K_{d}, K_{d}\right] \quad, \quad K_{j}=\frac{\pi}{L_{j}} .
$$

The spatial correlation length $L_{j}^{U}$ relative to the coordinate $x_{j}$ and defined by Eq. (9) is such that

$$
L_{j}^{U}=\int_{0}^{+\infty} \rho_{j}\left(\eta_{j}\right) d \eta_{j}=\frac{2 \pi}{2} s_{j}(0)=L_{j}
$$

Consequently, the parameters $L_{1}, \ldots, L_{d}$ represent the spatial correlation lengths of random field $U$. The autocorrelation function behavior at the origin is such that

$$
R_{U}(\boldsymbol{\eta})=1-\frac{\pi^{2}}{12}\left(\frac{\eta_{1}^{2}}{L_{1}^{2}}+\ldots+\frac{\eta_{d}^{2}}{L_{d}^{2}}\right)+o\left(\frac{\eta_{1}^{2}}{L_{1}^{2}}+\ldots+\frac{\eta_{d}^{2}}{L_{d}^{2}}\right) \quad \text { for } \quad\|\boldsymbol{\eta}\| \rightarrow 0
$$

Consequently, Eq. (6) is verified with $[C]$ the invertible diagonal $(d \times d)$ real matrix such that $[C]_{j j}=2 \sqrt{3} L_{j} / \pi$.

\subsubsection{Representation of the random field $U$ adapted to its numerical simulation}

In order to construct numerical simulations of random fields belonging to $\mathrm{SFG}^{+}$, we need to introduce an efficient representation of the germ $U$ in $\mathcal{E}_{U}$. The spatial discretization of this random field will directly be related to the spatial discretization of the elliptic stochastic partial differential operator for which the germ $U$ will be used. In general, the problem is setted on an arbitrary bounded domain $\Omega$ of $\mathbb{R}^{d}$ and the finite element method is utilized. Consequently, $U$ has to be simulated in $N$ given points $\mathbf{x}^{1}, \ldots, \mathbf{x}^{N}$ in $\Omega \subset \mathbb{R}^{d}$ (for instance located in the integrating points of the finite elements of the finite element mesh of domain $\Omega$ ). Clearly, the set of all these given points does not constitute a structured mesh corresponding to a constant spatial sampling step in each direction. This means that formulas based on the use of multidimensional Fast Fourier Transform (FFT) cannot be utilized. We present below two representations. The first one is adapted to the case for which $N$ is large and the second one is adapted to small or moderate value of $N$. With respect to the numerical cost, the most efficient representation is the second one, but this representation requires a large core memory. With respect to the core memory, the most efficient representation is the first one, but the numerical cost is higher than the second one.

(i) Representation adapted to a large value of $N$. The numerical simulation of homogeneous Gaussian vector-valued random field was introduced by Shinozuka [66-68]. A detailed development with additional mathematical properties related to convergence properties can be found in [54] and 
allows the following representation to be constructed. As an example, we consider the particular random field $U$ in $\mathcal{E}_{U}$ defined in Section 3.2.2 for which the power spectral density function is given by Eqs. (12) and (13). Let $\mathbf{V}=\left(U\left(\mathbf{x}^{1}\right), \ldots, U\left(\mathbf{x}^{N}\right)\right)$ be the $\mathbb{R}^{N}$-valued random variable corresponding to the spatial discretization of the random field $U$ in points $\mathbf{x}^{1}, \ldots, \mathbf{x}^{N}$ in $\mathbb{R}^{d}$. Let $\mathbf{X}^{1}, \ldots, \mathbf{X}^{d}$ be the $d$ vectors in $\mathbb{R}^{N}$ such that $\mathbf{X}^{j}=\left(x_{j}^{1}, \ldots, x_{j}^{N}\right)$ for $j=1, \ldots, d$. Below, if $\mathbf{W}=\left(W_{1}, \ldots, W_{N}\right)$ is a vector in $\mathbb{R}^{N}$ and, if $\alpha$ and $\beta$ are two real numbers, then the notation $\cos (\alpha+\beta \mathbf{W})$ means the vector in $\mathbb{R}^{N}$ whose components are $\left(\cos \left(\alpha+\beta W_{1}\right), \ldots, \cos \left(\alpha+\beta W_{N}\right)\right)$. Let $p \geq 1$ be an integer and let $\nu=2 p$. Let $\tau_{1}, \ldots, \tau_{\nu}$ be the sampling points of the interval $[-1,1]$ with the constant step $\Delta=2 / \nu$ such that

$$
\tau_{\beta}=-1+\left(\beta-\frac{1}{2}\right) \frac{2}{\nu}, \beta=1, \ldots, \nu .
$$

Let $S_{1}, \ldots, S_{\nu}$ be the positive real numbers defined by

$$
S_{\beta}=\frac{2}{\nu} q\left(\tau_{\beta}\right) \quad, \quad \beta=1, \ldots, \nu \quad,
$$

in which $\tau \mapsto q(\tau)$ is defined by Eq. (14). It should be noted that, for any $j=1, \ldots, d, S_{\beta}$ is equal to $\left(\pi \Delta / L_{j}\right) s_{j}\left(k_{j \beta}\right)$ in which $s_{j}\left(k_{j}\right)$ is defined by Eq. (13) and where $k_{j 1}, \ldots, k_{j \nu}$ are such that $k_{j \beta}=\pi \tau_{\beta} / L_{j}$ with $\beta=1, \ldots, \nu$. We then have the following vectorized $\nu$-order approximation $\mathbf{V}^{\nu}$ of the $\mathbb{R}^{N}$-valued Gaussian random variable $\mathbf{V}$,

$\mathbf{V}^{\nu}=\sum_{\beta_{1}=1}^{\nu} \ldots \sum_{\beta_{d}=1}^{\nu} \sqrt{2 S_{\beta_{1}} \times \ldots \times S_{\beta_{d}}} Z_{\beta_{1} \ldots \beta_{d}} \cos \left\{\Phi_{\beta_{1} \ldots \beta_{d}}+\frac{\pi}{L_{1}} \tau_{\beta_{1}} \mathbf{X}^{1}+\ldots+\frac{\pi}{L_{d}} \tau_{\beta_{d}} \mathbf{X}^{d}\right\}$

in which $Z_{\beta_{1} \ldots \beta_{d}}=\sqrt{-\log \Psi_{\beta_{1} \ldots \beta_{d}}}$ and where $\left\{\Phi_{\beta_{1} \ldots \beta_{d}}, \Psi_{\beta_{1} \ldots \beta_{d}}\right\}_{\beta_{1} \ldots \beta_{d}}$ is a set of independent random variables, $\Phi_{\beta_{1} \ldots \beta_{d}}$ being a uniform random variable on $[0,2 \pi]$ and $\Psi_{\beta_{1} \ldots \beta_{d}}$ being a uniform random variable on $[0,1]$.

For any $\nu=2 p$ fixed, $\mathbf{V}^{\nu}$ is a Gaussian vector and taking into account the properties of the random field $U$ given in Section 3.2.1, it can be proved [54] that:

(a) the covariance matrix of the random vector $\mathbf{V}^{\nu}$ defined by Eq. (18) converges to the covariance matrix of the random vector $\mathbf{V}$ when $\nu$ goes to infinity.

(b) the sequence $\left\{\mathbf{V}^{\nu}\right\}_{\nu}$ of Gaussian random vectors defined by Eq. (18) converges in law to the Gaussian random vector $\mathbf{V}$ when $\nu$ goes to infinity.

In practice, for fixed $d \geq 1, \nu$ can be chosen as $\nu^{d}=2^{m}$ with $m$ an integer such that $m=r d$ and consequently, we have $\nu=2^{m / d}$. Therefore, if $m=9$ then $\nu^{d}=512$ and thus $\nu=8$ for $d=3$ and $\nu=512$ for $d=1$.

C. Soize - CMAME - revised version December 2004 
(ii) Representation adapted to a small or moderate value of $N$. Let $U$ be any random field in $\mathcal{E}_{U}$. A direct representation could be used for constructing numerical simulations of the centered Gaussian random vector $\mathbf{V}$. The covariance matrix $\left[C_{\mathbf{V}}\right]$ in $\mathbb{M}_{N}^{+}(\mathbb{R})$ can easily be constructed by the formula

$$
\left[C_{\mathbf{V}}\right]_{i j}=R_{U}\left(\mathbf{x}^{i}-\mathbf{x}^{j}\right)
$$

Then, the Chowlesky factorization $\left[C_{\mathbf{V}}\right]=\left[L_{\mathbf{V}}\right]^{T}\left[L_{\mathbf{V}}\right]$ can be performed. Since $\mathbf{V}$ is a centered Gaussian vector, we can write

$$
\mathbf{V}=\left[L_{\mathbf{V}}\right]^{T} \tilde{\mathbf{V}}
$$

in which $\widetilde{\mathbf{V}}=\left(\widetilde{V}_{1}, \ldots, \widetilde{V}_{N}\right)$ is an $\mathbb{R}^{N}$-valued random variable whose components $\widetilde{V}_{1}, \ldots, \widetilde{V}_{N}$ are $N$ independent normalized Gaussian random variables $\left(E\left\{\widetilde{V}_{j}\right\}=0\right.$ and $E\left\{\widetilde{V}_{j}^{2}\right\}=1$ for $j=1, \ldots, N)$. Equation (20) allows numerical simulations to be easily constructed. However, such a formula required to construct matrix $\left[C_{\mathbf{V}}\right]$ which is a full $(N \times N)$ real matrix and requires to perform a complete Chowlesky factorization of a full matrix. Nevertheless, the Chowleskyfactorization step is processed outside the loop in which the construction of the realizations of the Monte Carlo method are constructed. This means that the problem is related to the core memory and not to the numerical cost. Finally, if the smaller diameter of domain $\Omega$ is very large with respect to spatial correlation lengths $L_{1}^{U} \ldots, L_{d}^{U}$, then a reduced representation of Eq. (20) can be constructed in computing the dominant eigensubspace of the positive-definite symmetric real matrix $\left[C_{\mathbf{V}}\right]$. In this case, the core memory problem disappears and the present method will be the most efficient.

\subsection{Definition of ensemble $\mathrm{SFG}^{+}$}

The ensemble $\mathrm{SFG}^{+}$with the germ $U$ belonging to $\mathcal{E}_{U}$ is defined as the set of all the random fields $\mathbf{x} \mapsto\left[\mathbf{G}_{n}(\mathbf{x})\right]$ defined on the probability space $(\Theta, \mathcal{T}, P)$, indexed by $\mathbb{R}^{d}$ where $d \geq 1$ is a fixed integer, with values in $\mathbb{M}_{n}^{+}(\mathbb{R})$ where $n \geq 2$ is another fixed integer and having a given mathematical structure which has to be defined. Before completing this definition, a one-parameter family of positive-valued function $u \mapsto h(\alpha, u)$ on $\mathbb{R}$ has to be defined and studied.

\subsubsection{Defining the family of functions $\{u \mapsto h(\alpha, u)\}_{\alpha>0}$}

Definition. Let $\alpha$ be a positive real number. The function $u \mapsto h(\alpha, u)$ from $\mathbb{R}$ into $] 0,+\infty[$ is such that $\Gamma_{\alpha}=h(\alpha, U)$ is a gamma random variable with parameter $\alpha$ while $U$ is a normalized Gaussian random variable $\left(E\{U\}=0\right.$ and $\left.E\left\{U^{2}\right\}=1\right)$. Consequently, for all $u$ in $\mathbb{R}$, we have

$$
h(\alpha, u)=F_{\Gamma_{\alpha}}^{-1}\left(F_{U}(u)\right)
$$


in which $u \mapsto F_{U}(u)=P(U \leq u)$ is the cumulative distribution function of the normalized Gaussian random variable $U$ such that

$$
F_{U}(u)=\int_{-\infty}^{u} \frac{1}{\sqrt{2 \pi}} e^{-t^{2} / 2} d t
$$

The function $p \mapsto F_{\Gamma_{\alpha}}^{-1}(p)$ from ]0,1[ into ]0, $+\infty[$ is the reciprocical function of the cumulative distribution function $\gamma \mapsto F_{\Gamma_{\alpha}}(\gamma)$ from $] 0,+\infty[$ into $] 0,1\left[\right.$ of the gamma random variable $\Gamma_{\alpha}$ with parameter $\alpha$, which is such that, for all $\gamma$ in $\mathbb{R}^{+}$,

$$
F_{\Gamma_{\alpha}}(\gamma)=\int_{0}^{\gamma} \frac{1}{\Gamma(\alpha)} t^{\alpha-1} e^{-t} d t
$$

in which $\Gamma(\alpha)=\int_{0}^{+\infty} t^{\alpha-1} e^{-t} d t$ is the gamma function [1].

Properties. For studying the mathematical properties of the random field $\mathbf{x} \mapsto\left[\mathbf{G}_{n}(\mathbf{x})\right]$, we need the following properties related to the family of functions $\{u \mapsto h(\alpha, u)\}_{\alpha>0}$. It can easily be proved that:

(i) For all $\alpha$ fixed in $] 0,+\infty[, u \mapsto h(\alpha, u)$ is a continuous function from $\mathbb{R}$ into $] 0,+\infty[$, which is such that

$$
h(\alpha, v)<h(\alpha, u) \quad, \quad \frac{1}{h(\alpha, u)}<\frac{1}{h(\alpha, v)} \quad, \quad \text { for all } \quad-\infty<v<u<+\infty \quad .
$$

(ii) For all $u$ fixed in $\mathbb{R}$, we have

$$
h(\beta, u)<h(\alpha, u) \quad, \quad \frac{1}{h(\alpha, u)}<\frac{1}{h(\beta, u)} \quad, \quad \text { for all } \quad 0<\beta<\alpha<+\infty \quad .
$$

(iii) Let $\alpha_{1}, \ldots, \alpha_{n}$ be the positive real numbers such that $\alpha_{1}>\alpha_{2}>\ldots>\alpha_{n}>0$. Figure 2 displays the graphs of functions $u \mapsto h\left(\alpha_{j}, u\right)$ and $u \mapsto 1 / h\left(\alpha_{j}, u\right)$.
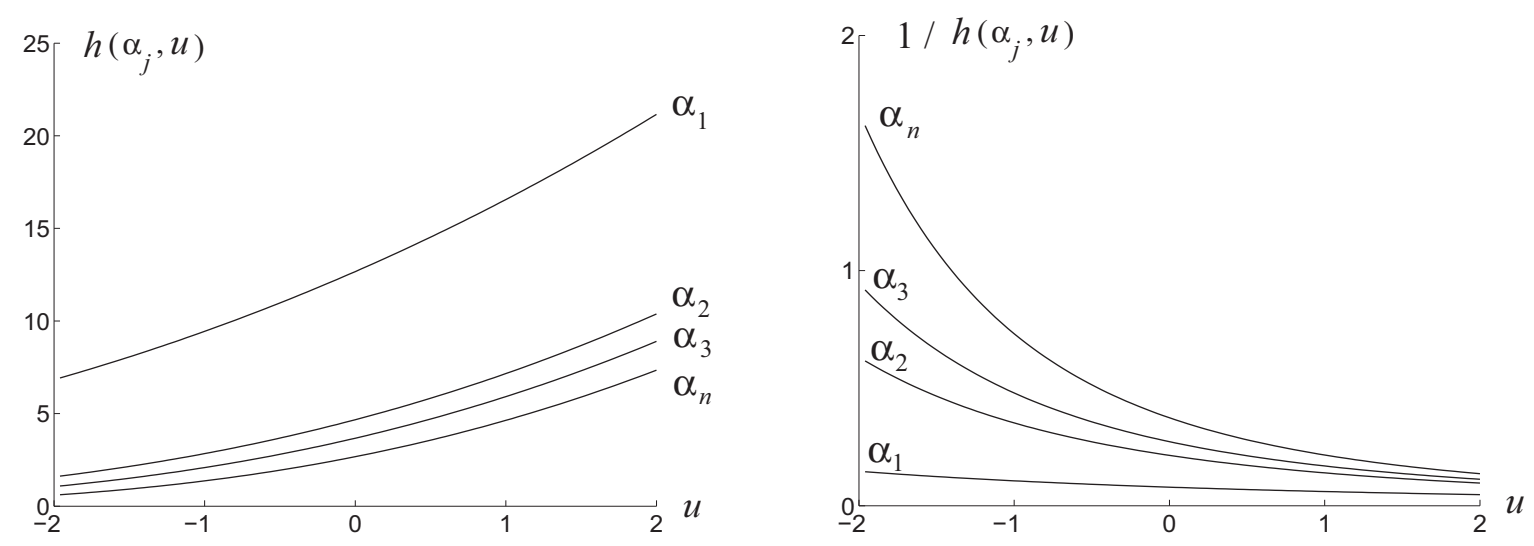

Fig. 2. Graph of function $u \mapsto h\left(\alpha_{j}, u\right)$ (left figure) and graph of function $u \mapsto 1 / h\left(\alpha_{j}, u\right)$ (right figure) for $\alpha_{1}>\alpha_{2}>\ldots>\alpha_{n}>0$. 
(iv) For $u \rightarrow-\infty$, we have the following asymptotic behavior,

$$
h(\alpha, u) \sim\left(\frac{\alpha \Gamma(\alpha)}{\sqrt{2 \pi}}\right)^{1 / \alpha} \frac{1}{(-u)^{1 / \alpha}} \exp \left\{-\frac{u^{2}}{2 \alpha}\right\} .
$$

Equation (26) is proved by using the two following results [1],

$$
\begin{aligned}
u \rightarrow-\infty \quad, \quad F_{U}(u) \sim-\frac{1}{u} \frac{1}{\sqrt{2 \pi}} \exp \left\{-\frac{u^{2}}{2}\right\} \quad, \\
\gamma \rightarrow 0 \quad, \quad F_{\Gamma_{\alpha}}(\gamma) \sim \frac{1}{\Gamma(\alpha)} \frac{1}{\alpha} \gamma^{\alpha} .
\end{aligned}
$$

\subsubsection{Defining the random field $\mathbf{x} \mapsto\left[\mathbf{G}_{n}(\mathbf{x})\right]$}

Let $U$ be a given random field belonging to $\mathcal{E}_{U}$ defined in Section 3.2.1 (for instance, the random field $U$ defined in Section 3.2.2).

(i) Let $\left\{U_{j j^{\prime}}(\mathbf{x}), \mathbf{x} \in \mathbb{R}^{d}\right\}_{1 \leq j \leq j^{\prime} \leq n}$ be $n(n+1) / 2$ independent copies of the random field $\{U(\mathbf{x}), \mathbf{x} \in$ $\left.\mathbb{R}^{d}\right\}$. Consequently, for all $\mathbf{x}$ in $\mathbb{R}^{d}$, we have

$$
E\left\{U_{j j^{\prime}}(\mathbf{x})\right\}=0 \quad, \quad E\left\{U_{j j^{\prime}}(\mathbf{x})^{2}\right\}=1 \quad, \quad 1 \leq j \leq j^{\prime} \leq n
$$

and for all $1 \leq j \leq j^{\prime} \leq n$, the random field $\mathbf{x} \mapsto U_{j j^{\prime}}(\mathbf{x})$ is completely defined.

(ii) Let $\delta$ be the real number, independent of $\mathbf{x}$ and $n$, such that

$$
0<\delta<\delta_{n} \quad, \quad \delta_{n}=\sqrt{(n+1)(n+5)^{-1}}<1 .
$$

This parameter will allow the dispersion of the random field to be controlled (see Section 3.4(v)).

(iii) The random field $\mathbf{x} \mapsto\left[\mathbf{G}_{n}(\mathbf{x})\right]$ is then defined as follows: for all $\mathbf{x}$ in $\mathbb{R}^{d}$,

$$
\left[\mathbf{G}_{n}(\mathbf{x})\right]=\left[\mathbf{L}_{n}(\mathbf{x})\right]^{T}\left[\mathbf{L}_{n}(\mathbf{x})\right],
$$

in which $\left[\mathbf{L}_{n}(\mathbf{x})\right]$ is an upper triangular random matrix with values in $\mathbb{M}_{n}(\mathbb{R})$ such that:

(a) The $n(n+1) / 2$ random fields $\mathbf{x} \mapsto\left[\mathbf{L}_{n}(\mathbf{x})\right]_{j j^{\prime}}$ for $1 \leq j \leq j^{\prime} \leq n$, are independent.

(b) For $j<j^{\prime}$, the real-valued random field $\mathbf{x} \mapsto\left[\mathbf{L}_{n}(\mathbf{x})\right]_{j j^{\prime}}$ indexed by $\mathbb{R}^{d}$ is defined by

$$
\left[\mathbf{L}_{n}(\mathbf{x})\right]_{j j^{\prime}}=\sigma_{n} U_{j j^{\prime}}(\mathbf{x})
$$

in which $\sigma_{n}$ is such that

$$
\sigma_{n}=\delta(n+1)^{-1 / 2}
$$


(c) For $j=j^{\prime}$, the positive-valued random field $\mathbf{x} \mapsto\left[\mathbf{L}_{n}(\mathbf{x})\right]_{j j}$ indexed by $\mathbb{R}^{d}$ is defined by

$$
\left[\mathbf{L}_{n}(\mathbf{x})\right]_{j j}=\sigma_{n} \sqrt{2 h\left(\alpha_{j}, U_{j j}(\mathbf{x})\right)}
$$

in which, for $j=1, \ldots, n$,

$$
\alpha_{j}=\frac{n+1}{2 \delta^{2}}+\frac{1-j}{2}
$$

and where $h(\alpha, u)$ is defined by Eq. (21). Equations (28) and (33) yield

$$
\alpha_{1}>\alpha_{2}>\ldots>\alpha_{n}>3
$$

\subsection{Basic properties of the random field $\mathbf{x} \mapsto\left[\mathbf{G}_{n}(\mathbf{x})\right]$}

The properties given in Sections (i) to (vi), (viii) and (xi) below are directly deduced from Refs. $[74,75]$. The other properties can easily be proved.

(i) Positiveness. For all $\mathbf{x}$ in $\mathbb{R}^{d}$, random matrix $\left[\mathbf{G}_{n}(\mathbf{x})\right]$ is a random variable with values in $M_{n}^{+}(\mathbb{R})$,

$$
\left[\mathbf{G}_{n}(\mathbf{x})\right] \in \mathbb{M}_{n}^{+}(\mathbb{R}) \text { a.s }
$$

(ii) Second-order random field. Random field $\mathbf{x} \mapsto\left[\mathbf{G}_{n}(\mathbf{x})\right]$ is second-order which means that

$$
E\left\{\left\|\left[\mathbf{G}_{n}(\mathbf{x})\right]\right\|_{F}^{2}\right\}<+\infty \quad, \quad \forall \mathbf{x} \in \mathbb{R}^{d}
$$

(iii) Homogeneousness. Random field $\mathbf{x} \mapsto\left[\mathbf{G}_{n}(\mathbf{x})\right]$ is homogeneous on $\mathbb{R}^{d}$ for the translation $\mathbf{x} \mapsto \mathbf{x}+\eta$ in $\mathbb{R}^{d}$.

(iv) Mean function. The mean function $\mathbf{x} \mapsto\left[\underline{G}_{n}(\mathbf{x})\right]=E\left\{\left[\mathbf{G}_{n}(\mathbf{x})\right]\right\}$ from $\mathbb{R}^{d}$ into $\mathbb{M}_{n}^{+}(\mathbb{R})$ is such that

$$
\left[\underline{G}_{n}(\mathbf{x})\right]=\left[I_{n}\right] \quad, \quad \forall \mathbf{x} \in \mathbb{R}^{d},
$$

in which $\left[I_{n}\right]$ is the identity matrix in $\mathbb{M}_{n}(\mathbb{R})$. 
(v) Dispersion parameter. Parameter $\delta$ which is independent of $\mathbf{x}$ and $n$ is such that

$$
\delta=\left\{\frac{E\left\{\left\|\left[\mathbf{G}_{n}(\mathbf{x})\right]-\left[\underline{G}_{n}(\mathbf{x})\right]\right\|_{F}^{2}\right\}}{\left\|\left[\underline{G}_{n}(\mathbf{x})\right]\right\|_{F}^{2}}\right\}^{1 / 2}=\left\{\frac{1}{n} E\left\{\left\|\left[\mathbf{G}_{n}(\mathbf{x})\right]-\left[I_{n}\right]\right\|_{F}^{2}\right\}\right\}^{1 / 2} .
$$

This equation shows that parameter $\delta$ allows the dispersion level of random field $\mathbf{x} \mapsto\left[\mathbf{G}_{n}(\mathbf{x})\right]$ to be controlled. From Eq. (37), it can be deduced that

$$
E\left\{\left\|\left[\mathbf{G}_{n}(\mathbf{x})\right]\right\|_{F}^{2}\right\}=n\left(\delta^{2}+1\right)
$$

Equation (38) is coherent with Eq. (35) and shows that $E\left\{\left\|\left[\mathbf{G}_{n}(\mathbf{x})\right]\right\|_{F}^{2}\right\} \rightarrow n$ when $\delta \rightarrow 0$. In fact, it can be proved that $\left[\mathbf{G}_{n}(\mathbf{x})\right] \rightarrow\left[I_{n}\right]$ in mean-square sense when $\delta \rightarrow 0$.

(vi) One-order marginal probability distribution. For all $\mathbf{x}$ fixed in $\mathbb{R}^{d}$, let $P_{\left[\mathbf{G}_{n}(\mathbf{x})\right]}$ be the probability distribution of random matrix $\left[\mathbf{G}_{n}(\mathbf{x})\right]$ (that is to say, the one-order marginal probability distribution of random field $\mathbf{x} \mapsto\left[\mathbf{G}_{n}(\mathbf{x})\right]$ ). From Refs. [74,75], it can directly be deduced that this probability distribution on $\mathbb{M}_{n}^{+}(\mathbb{R})$ is defined by a probability density function $\left[G_{n}\right] \mapsto p_{\left[\mathbf{G}_{n}(\mathbf{x})\right]}\left(\left[G_{n}\right]\right)$ from $\mathbb{M}_{n}^{+}(\mathbb{R})$ into $\mathbb{R}^{+}$, with respect to the measure $\widetilde{d} G_{n}$ on $M_{n}^{S}(\mathbb{R})$ defined by

$$
\widetilde{d} G_{n}=2^{n(n-1) / 4} \Pi_{1 \leq i \leq j \leq n} d\left[G_{n}\right]_{i j}
$$

in which $d\left[G_{n}\right]_{i j}$ is the Lebesgue measure on $\mathbb{R}$. We then have

$$
P_{\left[\mathbf{G}_{n}(\mathbf{x})\right]}=p_{\left[\mathbf{G}_{n}(\mathbf{x})\right]}\left(\left[G_{n}\right]\right) \widetilde{d} G_{n},
$$

with the usual normalization condition

$$
\int_{\mathbb{M}_{n}^{+}(\mathbb{R})} p_{\left[\mathbf{G}_{n}(\mathbf{x})\right]}\left(\left[G_{n}\right]\right) \tilde{d} G_{n}=1 .
$$

The probability density function $p_{\left[\mathbf{G}_{n}(\mathbf{x})\right]}\left(\left[G_{n}\right]\right)$ is then written as

$$
p_{\left[\mathbf{G}_{n}(\mathbf{x})\right]}\left(\left[G_{n}\right]\right)=\mathbb{1}_{\mathbb{M}_{n}^{+}(\mathbb{R})}\left(\left[G_{n}\right]\right) \times c_{n} \times\left(\operatorname{det}\left[G_{n}\right]\right)^{b_{n}} \times \exp \left\{-a_{n} \operatorname{tr}\left[G_{n}\right]\right\}
$$

in which $a_{n}=(n+1) /\left(2 \delta^{2}\right), b_{n}=a_{n}\left(1-\delta^{2}\right), \mathbb{1}_{\mathbb{M}_{n}^{+}(\mathbb{R})}\left(\left[G_{n}\right]\right)$ is equal to 1 if $\left[G_{n}\right] \in \mathbb{M}_{n}^{+}(\mathbb{R})$ and is equal to zero if $\left[G_{n}\right] \notin \mathbb{M}_{n}^{+}(\mathbb{R})$ and where positive constant $c_{n}$ is such that

$$
c_{n}=\frac{(2 \pi)^{-n(n-1) / 4} a_{n}^{n a_{n}}}{\Pi_{j=1}^{n} \Gamma\left(\alpha_{j}\right)},
$$

in which $\alpha_{j}$ is given by Eq. (33). Equation (42) clearly shows that, for all $\mathbf{x}$ in $\mathbb{R}^{d}$, the random variables $\left[\mathbf{G}_{n}(\mathbf{x})\right]_{i j}$ for all $1 \leq i \leq j \leq n$ are dependent. In addition, since $(n+1) / \delta^{2}$ is generally not an integer, then the probability distribution defined by Eqs. (40)-(43) is generally not a multivariate Wishart distribution $[4,17]$. 
(vii) System of marginal probability distributions. Let $u=\left\{u_{j j^{\prime}}, 1 \leq j \leq j^{\prime} \leq n\right\}$ be a vector in $\mathbb{R}^{\nu}$ with $\nu=n(n+1) / 2$. For all $\mathbf{x}$ fixed in $\mathbb{R}^{d}$, let $\mathbb{U}(\mathbf{x})=\left\{U_{j j^{\prime}}(\mathbf{x}), 1 \leq j \leq j^{\prime} \leq n\right\}$ be the Gaussian random vector with values in $\mathbb{R}^{\nu}$ in which $U_{j j^{\prime}}(\mathbf{x})$ is defined in Section 3.3.2(i). From Eqs. (29) to (33) and (21), it can be deduced that there exists a continuous nonlinear mapping $u \mapsto\left[g_{n}(u)\right]$ from $\mathbb{R}^{\nu}$ into $M_{n}^{+}(\mathbb{R})$ such that

$$
\left[\mathbf{G}_{n}(\mathbf{x})\right]=\left[g_{n}(\mathbb{U}(\mathbf{x}))\right] \quad, \quad \forall \mathbf{x} \in \mathbb{R}^{d}
$$

Let $\mathbf{x}^{1}, \ldots, \mathbf{x}^{N}$ be any finite and not ordered subset of $\mathbb{R}^{d}$. Then, the joint probability distribution on $\mathbb{M}_{n}^{+}(\mathbb{R}) \times \ldots \times \mathbb{M}_{n}^{+}(\mathbb{R})(N$ times $)$ of the random matrices $\left(\left[\mathbf{G}_{n}\left(\mathbf{x}^{1}\right)\right], \ldots,\left[\mathbf{G}_{n}\left(\mathbf{x}^{N}\right)\right]\right)$ is the image of the joint Gaussian probability distribution on $\mathbb{R}^{\nu} \times \ldots \times \mathbb{R}^{\nu}$ ( $N$ times) of the random vectors $\left(\mathbb{U}\left(\mathbf{x}^{1}\right), \ldots, \mathbb{U}\left(\mathbf{x}^{N}\right)\right)$ by the continuous nonlinear local mapping $\left(\mathfrak{u}^{1}, \ldots, \mathbb{U}^{N}\right) \mapsto\left[\mathcal{G}_{N}\left(\boldsymbol{u}^{1}, \ldots, \mathbb{U}^{N}\right)\right]$ from $\mathbb{R}^{\nu} \times \ldots \times \mathbb{R}^{\nu}$ into $M_{n}^{+}(\mathbb{R}) \times \ldots \times M_{n}^{+}(\mathbb{R})$ such that

$$
\left[\mathcal{G}_{N}\left(\mathfrak{u}^{1}, \ldots, \mathbb{u}^{N}\right)\right]=\left(\left[g_{n}\left(\boldsymbol{u}^{1}\right)\right], \ldots,\left[g_{n}\left(\mathfrak{u}^{N}\right)\right]\right) .
$$

Consequently, the system of the marginal probability distributions of random field $\mathbf{x} \mapsto\left[\mathbf{G}_{n}(\mathbf{x})\right]$ is well defined but cannot be explicitly calculated. Only the one-order marginal probability distribution is explicitly calculated (see Eqs. (40) to (43)). The random field $\mathbf{x} \mapsto\left[\mathbf{G}_{n}(\mathbf{x})\right]$ is non Gaussian. Nevertheless, since the nonlinear mapping $\left(\mathbb{u}^{1}, \ldots, \mathbb{U}^{N}\right) \mapsto\left[\mathcal{G}_{N}\left(\mathbb{u}^{1}, \ldots, \mathbb{u}^{N}\right)\right]$ is explicitly known, any realization $\left(\left[\mathbf{G}_{n}\left(\mathbf{x}^{1}, \theta\right)\right], \ldots,\left[\mathbf{G}_{n}\left(\mathbf{x}^{N}, \theta\right)\right]\right)$ for $\theta \in \Theta$ of random matrices $\left(\left[\mathbf{G}_{n}\left(\mathbf{x}^{1}\right)\right], \ldots,\left[\mathbf{G}_{n}\left(\mathbf{x}^{N}\right)\right]\right)$ can easily be computed by using Section 3.2.3.

(viii) Invertibility. There exists a positive constant $c_{0}$ independent of $n$ and independent of $\mathbf{x}$ but depending on $\delta$, such that

$$
E\left\{\left\|\left[\mathbf{G}_{n}(\mathbf{x})\right]^{-1}\right\|^{2}\right\} \leq c_{0}<+\infty \quad, \quad \forall n \geq 2 \quad, \quad \forall \mathbf{x} \in \mathbb{R}^{d} .
$$

From the inequality $\|A\|_{F} \leq \sqrt{n}\|A\|$ for $[A]$ in $M_{n}(\mathbb{R})$ (see Section 2), it can be deduced that

$$
E\left\{\left\|\left[\mathbf{G}_{n}(\mathbf{x})\right]^{-1}\right\|_{F}^{2}\right\} \leq c_{n}<+\infty \quad, \quad \forall n \geq 2 \quad, \quad \forall \mathbf{x} \in \mathbb{R}^{d},
$$

in which $c_{n}=n c_{0}$. Since $\left[\mathbf{G}_{n}(\mathbf{x})\right]$ belongs to $\mathbb{M}_{n}^{+}(\mathbb{R})$ almost surely, then $\left[\mathbf{G}_{n}(\mathbf{x})\right]^{-1}$ exists almost surely. However, since almost sure convergence does not imply mean-square convergence, then Eq. (46) cannot simply be deduced. In fact, Eq. (46) is proved by using Eq. (42) (see Ref. [75]) and means that $n \mapsto E\left\{\left\|\left[\mathbf{G}_{n}(\mathbf{x})\right]^{-1}\right\|^{2}\right\}$ is a bounded function (the proof of this result is difficult enough to perform). 
(ix) Mean-square continuity. Since function $u \mapsto\left[g_{n}(u)\right]$ defined by Eq. (44) is continuous from $\mathbb{R}^{\nu}$ into $\mathbb{M}_{n}^{+}(\mathbb{R})$ and since $\mathbf{x} \mapsto \mathbb{U}(\mathbf{x})$ is a mean-square continuous random field on $\mathbb{R}^{d}$ (see Section 3.2.2(i)), it can be deduced that random field $\mathbf{x} \mapsto\left[\mathbf{G}_{n}(\mathbf{x})\right]=\left[g_{n}(\mathbb{U}(\mathbf{x}))\right]$ is mean-square continuous on $\mathbb{R}^{d}$.

(x) Continuous trajectory. Since function $u \mapsto\left[g_{n}(u)\right]$ is continuous and since the trajectories of random field $\mathbf{x} \mapsto \mathbb{U}(\mathbf{x})$ are continuous from $\mathbb{R}^{d}$ into $\mathbb{R}^{\nu}$ almost surely (see Section 3.2.1), we directly deduce that the trajectories of random field $\mathbf{x} \mapsto\left[\mathbf{G}_{n}(\mathbf{x})\right]$ are continuous from $\mathbb{R}^{d}$ into $M_{n}^{+}(\mathbb{R})$ almost surely.

(xi) Fourth-order tensor-valued covariance function. Taking into account Eq. (36) and since the random field $\mathbf{x} \mapsto\left[\mathbf{G}_{n}(\mathbf{x})\right]$ is homogeneous and mean-square continuous on $\mathbb{R}^{d}$, the fourth-order tensor-valued covariance function $\boldsymbol{\eta} \mapsto C^{G_{n}}(\boldsymbol{\eta})$ defined for all $\boldsymbol{\eta}$ in $\mathbb{R}^{d}$ by

$$
\begin{aligned}
C^{G_{n}}(\boldsymbol{\eta}) & =E\left\{\left(\left[\mathbf{G}_{n}(\mathbf{x}+\boldsymbol{\eta})\right]-\left[\underline{G}_{n}(\mathbf{x}+\boldsymbol{\eta})\right]\right) \otimes\left(\left[\mathbf{G}_{n}(\mathbf{x})\right]-\left[\underline{G}_{n}(\mathbf{x})\right]\right)\right\} \\
& =E\left\{\left(\left[\mathbf{G}_{n}(\mathbf{x}+\boldsymbol{\eta})\right]-\left[I_{n}\right]\right) \otimes\left(\left[\mathbf{G}_{n}(\mathbf{x})\right]-\left[I_{n}\right]\right)\right\}
\end{aligned}
$$

does not depend on $\mathbf{x}$ and is a continuous function on $\mathbb{R}^{d}$. From [74], it can be deduced that the covariance tensor $C^{G_{n}}(0)$ of random matrix $\left[\mathbf{G}_{n}(\mathbf{x})\right]$ which is such that

$$
C_{j k, j^{\prime} k^{\prime}}^{G_{n}}(0)=E\left\{\left(\left[\mathbf{G}_{n}(\mathbf{x})\right]_{j k}-\left[I_{n}\right]_{j k}\right)\left(\left[\mathbf{G}_{n}(\mathbf{x})\right]_{j^{\prime} k^{\prime}}-\left[I_{n}\right]_{j^{\prime} k^{\prime}}\right)\right\}
$$

is written as

$$
C_{j k, j^{\prime} k^{\prime}}^{G_{n}}(0)=\frac{\delta^{2}}{n+1}\left\{\left[I_{n}\right]_{j^{\prime} k}\left[I_{n}\right]_{j k^{\prime}}+\left[I_{n}\right]_{j j^{\prime}}\left[I_{n}\right]_{k k^{\prime}}\right\}
$$

In particular, the variance $V_{j k}^{G_{n}}$ of the random variable $\left[\mathbf{G}_{n}(\mathbf{x})\right]_{j k}$ is such that

$$
V_{j k}^{G_{n}}=C_{j k, j k}^{G_{n}}(0)=\frac{\delta^{2}}{n+1}\left(1+\left[I_{n}\right]_{j k}\right)
$$

(xii) Fourth-order tensor-valued spectral measure. Since random field $\mathbf{x} \mapsto\left[\mathbf{G}_{n}(\mathbf{x})\right]$ is homogeneous and mean-square continuous on $\mathbb{R}^{d}$, there is a fourth-order tensor-valued spectral measure $M^{G_{n}}(d \mathbf{k})$ on $\mathbb{R}^{d}$ which is such that, for all $\boldsymbol{\eta}$ in $\mathbb{R}^{d}$,

$$
C^{G_{n}}(\boldsymbol{\eta})=\int_{\mathbb{R}^{d}} e^{i<\eta, \mathbf{k}>} M^{G_{n}}(d \mathbf{k})
$$


It can be verified that the function $\boldsymbol{\eta} \mapsto C^{G_{n}}(\boldsymbol{\eta})$ is integrable on $\mathbb{R}^{d}$. Therefore, we can write $M^{G_{n}}(d \mathbf{k})=S^{G_{n}}(\mathbf{k}) d \mathbf{k}$ in which the fourth-order tensor-valued spectral density function $\mathbf{k} \mapsto$ $S^{G_{n}}(\mathbf{k})$ is a continuous function on $\mathbb{R}^{d}$ given by

$$
S^{G_{n}}(\mathbf{k})=\frac{1}{(2 \pi)^{d}} \int_{\mathbb{R}^{d}} e^{-i<\mathbf{k}, \boldsymbol{\eta}>} C^{G_{n}}(\boldsymbol{\eta}) d \boldsymbol{\eta}
$$

(xiii) Spatial correlation lengths. Let $\boldsymbol{\eta}=\left(\eta_{1}, \ldots, \eta_{d}\right) \mapsto r^{G_{n}}(\boldsymbol{\eta})$ be the function defined from $\mathbb{R}^{d}$ into $\mathbb{R}$ by

$$
\begin{aligned}
r^{G_{n}}(\boldsymbol{\eta}) & =\frac{1}{\delta^{2}\left\|\left[\underline{G}_{n}(\mathbf{x})\right]\right\|_{F}^{2}} \operatorname{tr} E\left\{\left(\left[\mathbf{G}_{n}(\mathbf{x}+\boldsymbol{\eta})\right]-\left[\underline{G}_{n}(\mathbf{x}+\boldsymbol{\eta})\right]\right)\left(\left[\mathbf{G}_{n}(\mathbf{x})\right]-\left[\underline{G}_{n}(\mathbf{x})\right]\right)\right\} \\
& =\frac{1}{\delta^{2}}\left\{\frac{1}{n} E\left\{\operatorname{tr}\left\{\left[\mathbf{G}_{n}(\mathbf{x}+\boldsymbol{\eta})\right]\left[\mathbf{G}_{n}(\mathbf{x})\right]\right\}\right\}-1\right\} .
\end{aligned}
$$

Clearly (in particular due to Eq. (38)), it can be seen that

$$
r^{G_{n}}(0)=1 \quad, \quad r^{G_{n}}(-\boldsymbol{\eta})=r^{G_{n}}(\boldsymbol{\eta})
$$

For all $j=1, \ldots, d$, the spatial correlation length $L_{j}^{G_{n}}$ of random field $\mathbf{x} \mapsto\left[\mathbf{G}_{n}(\mathbf{x})\right]$, relative to coordinate $x_{j}$, can be defined by

$$
L_{j}^{G_{n}}=\int_{0}^{+\infty}\left|r^{G_{n}}\left(0, \ldots, 0, \eta_{j}, 0, \ldots, 0\right)\right| d \eta_{j} .
$$

Example. Let us consider the case $n=6, d=1, \delta=0.5, L_{1}=1$ in which $L_{1}$ is defined in Section 3.2.1. In Eq. (54), the mathematical expectation is estimated with 20000 realizations and Eq. (20) is used with $N=100$. The correlation length $L_{1}^{G_{n}}$ is calculated with Eq. (55) in approximating the interval $\left[0,+\infty\right.$ [ by the interval $\left[0,20\left[\right.\right.$ and yields $L_{1}^{G_{n}}=1.0614$. Figure 3 displays the graph of the function $\eta_{1} \mapsto r^{G_{n}}\left(\eta_{1}\right)$.

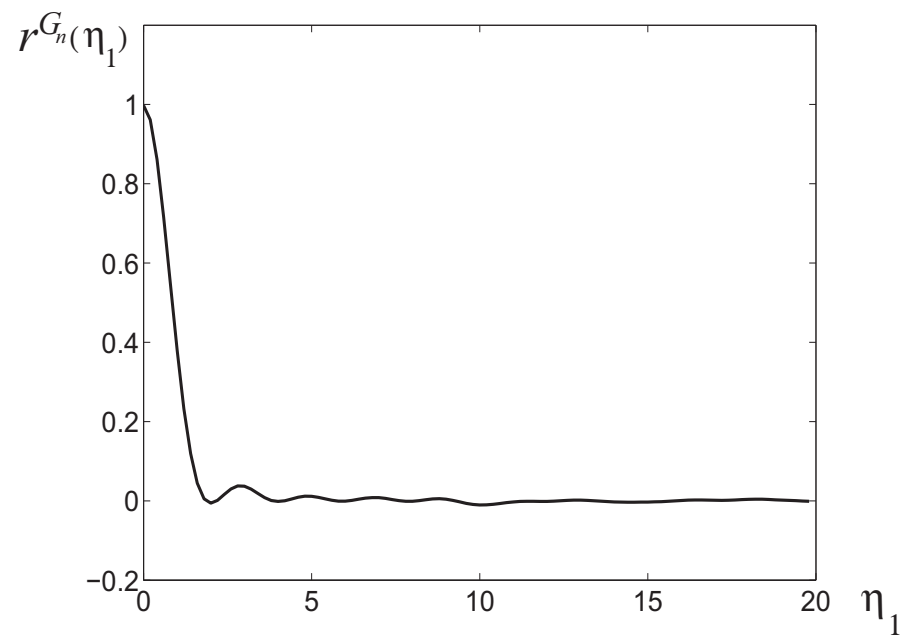

Fig. 3. Graph of function $\eta_{1} \mapsto r^{G_{n}}\left(\eta_{1}\right)$ for the case $n=6, d=1, \delta=0.5, L_{1}=1$. 
(xiv) Independence of the construction with respect to the choice of the Cartesian coordinate systems. In order to prove the independence of the proposed construction of the random field $\mathbf{x} \mapsto\left[\mathbf{G}_{n}(\mathbf{x})\right]$ with respect to the choice of the Cartesian coordinate system, we have to prove that, for all $\mathbf{x}$ fixed in $\mathbb{R}^{d}$, the probability distribution of random matrix $\left[\mathbf{G}_{n}(\mathbf{x})\right]$ is invariant under real orthogonal transformations. Let $\left[\Phi_{n}\right]$ be any real orthogonal matrix belonging to $\mathbb{M}_{n}(\mathbb{R})$ such that $\left[\Phi_{n}\right]^{T}\left[\Phi_{n}\right]=\left[\Phi_{n}\right]\left[\Phi_{n}\right]^{T}=\left[I_{n}\right]$. Let $\left[\mathbf{G}_{n}^{\prime}(\mathbf{x})\right]$ be the random matrix with values in $\mathbb{M}_{n}^{+}(\mathbb{R})$ defined by $\left[\mathbf{G}_{n}^{\prime}(\mathbf{x})\right]=\left[\Phi_{n}\right]^{T}\left[\mathbf{G}_{n}(\mathbf{x})\right]\left[\Phi_{n}\right]$. We then have

$$
\left[\mathbf{G}_{n}(\mathbf{x})\right]=\left[\Phi_{n}\right]\left[\mathbf{G}_{n}^{\prime}(\mathbf{x})\right]\left[\Phi_{n}\right]^{T}
$$

The probability density function $p_{\left[\mathbf{G}_{n}^{\prime}(\mathbf{x})\right]}\left(\left[G_{n}^{\prime}\right]\right)$ of random matrix $\left[\mathbf{G}_{n}^{\prime}(\mathbf{x})\right]$ with respect to the volume element $\widetilde{d} G_{n}^{\prime}$ (see Eq. (39)) is such that

$$
p_{\left[\mathbf{G}_{n}^{\prime}(\mathbf{x})\right]}\left(\left[G_{n}^{\prime}\right]\right) \tilde{d} G_{n}^{\prime}=p_{\left[\mathbf{G}_{n}(\mathbf{x})\right]}\left(\left[G_{n}\right]\right) \tilde{d} G_{n},
$$

in which $p_{\left[\mathbf{G}_{n}(\mathbf{x})\right]}\left(\left[G_{n}\right]\right)$ is defined by Eq. (42). Let $\left[G_{n}\right]$ and $\left[G_{n}^{\prime}\right]$ be such that $\left[G_{n}\right]=$ $\left[\Phi_{n}\right]\left[G_{n}^{\prime}\right]\left[\Phi_{n}\right]^{T}$. Since $\left[\Phi_{n}\right]$ is a real orthogonal matrix, it can be deduced that $\widetilde{d} G_{n}=\widetilde{d} G_{n}^{\prime}$, $\operatorname{det}\left[G_{n}\right]=\operatorname{det}\left[G_{n}^{\prime}\right]$ and $\operatorname{tr}\left[G_{n}\right]=\operatorname{tr}\left[G_{n}^{\prime}\right]$. From Eq. (42), we deduce that

$$
p_{\left[\mathbf{G}_{n}(\mathbf{x})\right]}\left(\left[G_{n}\right]\right) \widetilde{d} G_{n}=p_{\left[\mathbf{G}_{n}(\mathbf{x})\right]}\left(\left[G_{n}^{\prime}\right]\right) \widetilde{d} G_{n}^{\prime},
$$

and consequently, we have

$$
p_{\left[\mathbf{G}_{n}^{\prime}(\mathbf{x})\right]}\left(\left[G_{n}^{\prime}\right]\right) \widetilde{d} G_{n}^{\prime}=p_{\left[\mathbf{G}_{n}(\mathbf{x})\right]}\left(\left[G_{n}^{\prime}\right]\right) \widetilde{d} G_{n}^{\prime},
$$

which proves the invariance of random matrix $\left[\mathbf{G}_{n}(\mathbf{x})\right]$ under real orthogonal transformations.

\subsection{Fundamental property of the random field $\mathbf{x} \mapsto\left[\mathbf{G}_{n}(\mathbf{x})\right]$}

Let $\Omega$ be a bounded open domain of $\mathbb{R}^{d}$ and let $\bar{\Omega}=\Omega \cup \partial \Omega$ be its closure in which $\partial \Omega$ is the boundary of $\Omega$. We then have

$$
E\left\{\left(\sup _{\mathbf{x} \in \bar{\Omega}}\left\|\left[\mathbf{G}_{n}(\mathbf{x})\right]^{-1}\right\|\right)^{2}\right\}=c_{G}^{2}<+\infty
$$

in which sup is the supremum and where $0<c_{G}<+\infty$ is a finite positive constant.

Remark. Let us consider the case $d=1$ with $\bar{\Omega}$ be a compact interval of $\mathbb{R}$. Since the stochastic process $\left\{\left\|G_{n}(\mathbf{x})^{-1}\right\|, \mathbf{x} \in \bar{\Omega} \subset \mathbb{R}\right\}$ is not a continuous local martingal with respect 
to an increasing family of $\sigma$-fields, the following fundamental Doob maximal inequality $[15,53]$, $E\left\{\sup _{\mathbf{x} \in \bar{\Omega}}\left\|\left[\mathbf{G}_{n}(\mathbf{x})\right]^{-1}\right\|^{2}\right\} \leq 4 E\left\{\left\|\left[\mathbf{G}_{n}(\mathbf{x})\right]^{-1}\right\|^{2}\right\}$ cannot be used. In addition, we have to consider the non Gaussian random field case $d \geq 2$. Consequently, there is no known result allowing a direct proof of Eq. (56) to be obtained and a proof of this fundamental result has to be given.

Proof of Eq. (56). Let $\mathbf{x}$ be any point fixed in $\mathbb{R}^{d}$. The random eigenvalues of the random upper triangular matrix $\left[\mathbf{L}_{n}(\mathbf{x})\right]$ are $\left[\mathbf{L}_{n}(\mathbf{x})\right]_{11}, \ldots,\left[\mathbf{L}_{n}(\mathbf{x})\right]_{n n}$. Thus, the random eigenvalues of the random matrix $\left[\mathbf{L}_{n}(\mathbf{x})\right]^{-1}$ are $1 /\left[\mathbf{L}_{n}(\mathbf{x})\right]_{11}, \ldots, 1 /\left[\mathbf{L}_{n}(\mathbf{x})\right]_{n n}$ and, since $\left[\mathbf{L}_{n}(\mathbf{x})\right]_{j j}$ is a positive-valued random variable, then the operator norm $\left\|\left[\mathbf{L}_{n}(\mathbf{x})\right]^{-1}\right\|$ of random matrix $\left[\mathbf{L}_{n}(\mathbf{x})\right]^{-1}$ can be written as $\left\|\left[\mathbf{L}_{n}(\mathbf{x})\right]^{-1}\right\|=\sup _{j=1, \ldots, n}\left\{1 /\left[\mathbf{L}_{n}(\mathbf{x})\right]_{j j}\right\}$. Using Eq. (32) yields $\left\|\left[\mathbf{L}_{n}(\mathbf{x})\right]^{-1}\right\|^{2}=\frac{1}{2 \sigma_{n}^{2}} \sup _{j=1, \ldots, n}\left\{1 / h\left(\alpha_{j}, U_{j j}(\mathbf{x})\right)\right\}$. From Eq. (29), we deduce that $\left[\mathbf{G}_{n}(\mathbf{x})\right]^{-1}=$ $\left[\mathbf{L}_{n}(\mathbf{x})\right]^{-1}\left[\mathbf{L}_{n}(\mathbf{x})\right]^{-T}$ and consequently, $\left\|\left[\mathbf{G}_{n}(\mathbf{x})\right]^{-1}\right\| \leq\left\|\left[\mathbf{L}_{n}(\mathbf{x})\right]^{-1}\right\|^{2}$. We can then conclude that, for all $\mathbf{x}$ fixed in $\mathbb{R}^{d}$,

$$
\left\|\left[\mathbf{G}_{n}(\mathbf{x})\right]^{-1}\right\| \leq \frac{1}{2 \sigma_{n}^{2}} \sup _{j=1, \ldots, n}\left\{\frac{1}{h\left(\alpha_{j}, U_{j j}(\mathbf{x})\right)}\right\} .
$$

For all $j=1, \ldots, n$, let $\operatorname{Inf}_{j}$ be the real-valued random variable defined by

$$
\operatorname{Inf}_{j}=\inf _{\mathbf{x} \in \bar{\Omega}} U_{j j}(\mathbf{x})
$$

From Eq. (34), we deduce that, for all $j=1, \ldots, n-1$, we have $\alpha_{j}>\alpha_{n}$ and using Eq. (25) yields $1 / h\left(\alpha_{j}, U_{j j}(\mathbf{x})\right)<1 / h\left(\alpha_{n}, U_{j j}(\mathbf{x})\right)$. Finally, from Eqs. (24) and (58), it can be deduced that,

$$
\forall \mathbf{x} \in \bar{\Omega} \quad, \quad \forall j=1, \ldots n \quad, \quad \frac{1}{h\left(\alpha_{j}, U_{j j}(\mathbf{x})\right)} \leq \frac{1}{h\left(\alpha_{n}, \operatorname{Inf}_{j}\right)} \quad .
$$

Equations (57) and (59) yield

$$
\left(\sup _{\mathbf{x} \in \bar{\Omega}}\left\|\left[\mathbf{G}_{n}(\mathbf{x})\right]^{-1}\right\|\right)^{2} \leq \sup \left\{B_{1}, \ldots, B_{n}\right\}
$$

in which, for all $j=1, \ldots, n, B_{j}$ is the positive-valued random variable defined by

$$
B_{j}=\frac{1}{4 \sigma_{n}^{4}} \frac{1}{h\left(\alpha_{n}, \operatorname{Inf}_{j}\right)^{2}} .
$$

Since random fields $U_{11}, \ldots, U_{n n}$ are independent copies of random field $U$ (see Section 3.3.2), from Eq. (58), we deduce that $\operatorname{Inf}_{1}, \ldots, \operatorname{Inf}_{n}$ are $n$ independent copies of the real-valued random variable Inf defined by

$$
\operatorname{Inf}=\inf _{\mathbf{x} \in \bar{\Omega}} U(\mathbf{x})
$$


in which $U$ is the random field defined in Section 3.2.1. Therefore, random variables $B_{1}, \ldots, B_{n}$ are $n$ independent copies of the positive-valued random variable $B$ defined by

$$
B=\frac{1}{4 \sigma_{n}^{4}} \frac{1}{h\left(\alpha_{n}, \text { Inf }\right)^{2}} .
$$

Let $F_{B}(b)=P\{B \leq b\}$ be the cumulative distribution function of random variable $B$. Let $M$ be the positive-valued random variable defined by

$$
M=\sup \left\{B_{1}, \ldots, B_{n}\right\}
$$

Therefore, the cumulative distribution function $F_{M}(b)=P\{M \leq b\}$ of random variable $M$ is such that $F_{M}(b)=P\left\{B_{1} \leq b, \ldots, B_{n} \leq b\right\}=F_{B}(b)^{n}$ because $B_{1}, \ldots, B_{n}$ are $n$ independent copies of random variable $B$. We then have $E\{M\}=\int_{0}^{+\infty} b d F_{M}(b)=n \int_{0}^{+\infty} b F_{B}(b)^{n-1} d F_{B}(b)$ and since $F_{B}(b) \leq 1$, we have $F_{B}(b)^{n-1} \leq 1$ and consequently, $E\{M\} \leq n E\{B\}$. Using Eqs. (60) and (64), it can be deduced that

$$
E\left\{\left(\sup _{\mathbf{x} \in \bar{\Omega}}\left\|\left[\mathbf{G}_{n}(\mathbf{x})\right]^{-1}\right\|\right)^{2}\right\} \leq n E\{B\}
$$

Let $p_{\operatorname{Inf}}(u)$ be the probability density function with respect to $d u$ of the real-valued random variable Inf defined by Eq. (62). From Eq. (63), it is deduced that

$$
E\{B\}=\frac{1}{4 \sigma_{n}^{4}} \int_{\mathbb{R}} \frac{1}{h\left(\alpha_{n}, u\right)^{2}} p_{\operatorname{Inf}}(u) d u
$$

Clearly, since $u \mapsto 1 / h\left(\alpha_{n}, u\right)^{2}$ is a positive-valued decreasing function (see Eq. (24)), if for $b \gg 1$,

$$
H_{b}=\int_{-\infty}^{-b} \frac{1}{h\left(\alpha_{n}, u\right)^{2}} p_{\operatorname{Inf}}(u) d u,
$$

is finite, then we will have proved that Eq. (56) holds. Therefore, if $H_{b}<+\infty$ for $b \gg 1$, then the proof will be complete. Since $\mathbf{x} \mapsto U(\mathbf{x})$ is a centered Gaussian field, the random field $\mathbf{x} \mapsto-U(\mathbf{x})$ is isonomic to random field $\mathbf{x} \mapsto U(\mathbf{x})$. It can then easily be verified that the probability density function $u \mapsto p_{\text {Sup }}(u)$ with respect to $d u$ of the random variable

$$
\operatorname{Sup}=\sup _{\mathbf{x} \in \bar{\Omega}} U(\mathbf{x})
$$

is such that $p_{\operatorname{Sup}}(u)=p_{\operatorname{Inf}}(-u)$. Then, Eq. (67) can be rewritten as

$$
H_{b}=\int_{b}^{+\infty} \frac{1}{h\left(\alpha_{n},-u\right)^{2}} p_{\operatorname{Sup}}(u) d u
$$


Since $U$ is a Gaussian random field on $\mathbb{R}^{d}$, some results concerning the probability distribution of random variable Sup can be found in Refs. [2,3,5,12,18,53,90]. Taking into account the property defined by Eq. (6), we have (see Ref. [53], page 106), $P\{\operatorname{Sup}>u\}_{u \rightarrow+\infty} \sim c u^{3} \psi(u)(1+o(1)$ ), in which $c$ is a positive constant and where $\psi(u)=\int_{u}^{+\infty} p(t) d t$ with $p(t)=(2 \pi)^{-1 / 2} \exp \left(-t^{2} / 2\right)$. For $u \rightarrow+\infty$, we have [1], $\psi(u) \sim u^{-1} p(u)$ and thus,

$$
P\{\operatorname{Sup}>u\}_{u \rightarrow+\infty} \sim c u^{2} p(u)
$$

Let $\psi_{\operatorname{Sup}}(u)=P\{\operatorname{Sup}>u\}$. Since $d \psi_{\operatorname{Sup}}(u)=-p_{\text {Sup }}(u) d u$, Eq. (69) can be rewritten as $H_{b}=-\int_{b}^{+\infty} f(u) d \psi_{\operatorname{Sup}}(u) d u$ in which $f(u)=h\left(\alpha_{n},-u\right)^{-2}$. Since $\alpha_{n}>2$ (see Eq. (34)) and from Eq. (26), it can be deduced that $\lim _{u \rightarrow+\infty}\left\{f(u) \psi_{\text {Sup }}(u)\right\}=0$ and that, for all $\beta>0$, $\lim _{u \rightarrow+\infty}\left\{u^{\beta} f(u) p(u)\right\}=0$. An integration by parts yields $H_{b}=f(b) \psi_{\text {Sup }}(b)+\int_{b}^{+\infty} \psi_{\text {Sup }}(u)$ $d f(u)$. Using Eq. (70), we deduce that if $b \rightarrow+\infty$ then for $u \in\left[b,+\infty\left[\right.\right.$, we have $\psi_{\text {Sup }}(u) \sim$ $c u^{2} p(u)$ and thus, $H_{b} \sim f(b) \psi_{\text {Sup }}(b)+c \int_{b}^{+\infty} u^{2} p(u) d f(u)$. A new integration by part allows us to write, for $b \rightarrow+\infty, H_{b} \sim f(b) \psi_{\text {Sup }}(b)-c b^{2} f(b) p(b)-2 c \int_{b}^{+\infty} u f(u) p(u) d u+$ $c \int_{b}^{+\infty} u^{3} f(u) p(u) d u$. Clearly, $H_{b}$ will be finite for $b \rightarrow+\infty$, if $\widetilde{H}_{b}=\int_{b}^{+\infty} u^{3} f(u) p(u) d u$ is finite. Using Eq. (26) and since $\alpha_{n}>2$, we deduce that $\widetilde{H}_{b}$ is finite for $b \rightarrow+\infty$. This completes the proof.

\section{Construction and properties of the ensemble $\mathrm{SFE}^{+}$of non Gaussian positive-definite matrix- valued random fields}

\subsection{Definition of the ensemble $\mathrm{SFE}^{+}$}

Let $d \geq 1$ and $n \geq 2$ be two fixed integers. Let $\Omega$ be an open (or closed) bounded (or not) domain of $\mathbb{R}^{d}$ (we can have $\Omega=\mathbb{R}^{d}$ ). Let $\mathbf{x} \mapsto\left[\underline{a}_{n}(\mathbf{x})\right]$ be a matrix-valued field from $\Omega$ into $M_{n}^{+}(\mathbb{R})$, i.e.,

$$
\forall \mathbf{x} \in \Omega \subset \mathbb{R}^{d} \quad, \quad\left[\underline{a}_{n}(\mathbf{x})\right] \in \mathbb{M}_{n}^{+}(\mathbb{R})
$$

Then, for all $\mathbf{x}$ fixed in $\Omega$, there is an upper triangular invertible matrix $\left[\underline{L}_{n}(\mathbf{x})\right]$ in $\mathbb{M}_{n}(\mathbb{R})$ corresponding to the Cholesky factorization [27] of matrix $\left[\underline{a}_{n}(\mathbf{x})\right]$, such that

$$
\left[\underline{a}_{n}(\mathbf{x})\right]=\left[\underline{L}_{n}(\mathbf{x})\right]^{T}\left[\underline{L}_{n}(\mathbf{x})\right]
$$

It is assumed that the matrix-valued field $\mathbf{x} \mapsto\left[\underline{a}_{n}(\mathbf{x})\right]$ defined on $\Omega$ is such that: 
(i) There is a real positive constant $0<\underline{c}_{0}<+\infty$ independent of $\mathbf{x}$ such that, for all $\mathbf{x}$ in $\Omega$,

$$
\left.\forall \mathbf{y} \in \mathbb{R}^{n} \quad, \quad<\underline{a}_{n}(\mathbf{x})\right] \mathbf{y}, \mathbf{y}>\geq \underline{c}_{0}\|\mathbf{y}\|^{2}
$$

(ii) There is a real positive constant $0<\underline{c}_{1}<+\infty$ independent of $\mathbf{x}$ such that

$$
\forall \mathbf{x} \in \Omega \quad, \quad \sqrt{\underline{c}_{0}} \leq\left\|\left[\underline{L}_{n}(\mathbf{x})\right]\right\| \leq \sqrt{\underline{c}_{1}},
$$

the left inequality resulting from Eqs. (72) and (73). For all $\mathbf{y}$ in $\mathbb{R}^{n}$, we have $<\left[\underline{a}_{n}(\mathbf{x})\right] \mathbf{y}, \mathbf{y}>=$ $\left\|\left[\underline{L}_{n}(\mathbf{x})\right] \mathbf{y}\right\|^{2} \leq\left\|\left[\underline{L}_{n}(\mathbf{x})\right]\right\|^{2}\|\mathbf{y}\|^{2}$ and consequently, for all $\mathbf{x}$ in $\Omega$,

$$
\left.\forall \mathbf{y} \in \mathbb{R}^{n} \quad, \quad<\underline{a}_{n}(\mathbf{x})\right] \mathbf{y}, \mathbf{y}>\leq \underline{c}_{1}\|\mathbf{y}\|^{2} .
$$

(iii) It should be noted that $\left\|\left[\underline{a}_{n}(\mathbf{x})\right]\right\| \leq\left\|\left[\underline{L}_{n}(\mathbf{x})\right]\right\|^{2}$ and thus, using Eq. (74) and the inequality $\left\|\left[\underline{a}_{n}(\mathbf{x})\right]\right\|_{F} \leq \sqrt{n}\left\|\left[\underline{a}_{n}(\mathbf{x})\right]\right\|$ yield

$$
\forall \mathbf{x} \in \Omega \quad, \quad\left\|\left[\underline{a}_{n}(\mathbf{x})\right]\right\| \leq \underline{c}_{1} \quad, \quad\left\|\left[\underline{a}_{n}(\mathbf{x})\right]\right\|_{F} \leq \sqrt{n} \underline{c}_{1}
$$

Equation (76) means that the matrix-valued field $\mathbf{x} \mapsto\left[\underline{a}_{n}(\mathbf{x})\right]$ is a bounded function on $\Omega$.

(iv) The ensemble $\mathrm{SFE}^{+}$is then defined as the set of all the random fields $\mathbf{x} \mapsto\left[\mathbf{A}_{n}(\mathbf{x})\right]$ defined on probability space $(\Theta, \mathcal{T}, P)$, indexed by $\Omega$, with values in $M_{n}^{+}(\mathbb{R})$, such that

$$
\forall \mathbf{x} \in \Omega \quad, \quad\left[\mathbf{A}_{n}(\mathbf{x})\right]=\left[\underline{L}_{n}(\mathbf{x})\right]^{T}\left[\mathbf{G}_{n}(\mathbf{x})\right]\left[\underline{L}_{n}(\mathbf{x})\right] \quad,
$$

in which $\mathbf{x} \mapsto\left[\mathbf{G}_{n}(\mathbf{x})\right]$ is the random field in $\mathrm{SFG}^{+}$defined on $(\Theta, \mathcal{T}, P)$, indexed by $\mathbb{R}^{d}$ and with values in $M_{n}^{+}(\mathbb{R})$ (see Section 3.3.2).

\subsection{Basic properties of the random field $\mathbf{x} \mapsto\left[\mathbf{A}_{n}(\mathbf{x})\right]$}

The following properties are directly deduced from Sections 3.4 and 4.1.

(i) Positiveness. For all $\mathbf{x}$ in $\Omega,\left[\mathbf{A}_{n}(\mathbf{x})\right]$ is a random matrix with values in $\mathbb{M}_{n}^{+}(\mathbb{R})$, i.e.

$$
\left[\mathbf{A}_{n}(\mathbf{x})\right] \in \mathbb{M}_{n}^{+}(\mathbb{R}) \text { a.s }
$$


(ii) Second-order random field. From Eq. (77), we deduce that $\left\|\left[\mathbf{A}_{n}(\mathbf{x})\right]\right\| \leq\left\|\left[\underline{L}_{n}(\mathbf{x})\right]\right\|^{2}\left\|\left[\mathbf{G}_{n}(\mathbf{x})\right]\right\|$ and consequently, from the inequalities $\|A\| \leq\|A\|_{F} \leq \sqrt{n}\|A\|$, it can be written that

$$
\left\|\left[\mathbf{A}_{n}(\mathbf{x})\right]\right\|_{F}^{2} \leq n\left\|\left[\underline{L}_{n}(\mathbf{x})\right]\right\|^{4}\left\|\left[\mathbf{G}_{n}(\mathbf{x})\right]\right\|_{F}^{2}
$$

Using Eqs. (35) and (74) yields

$$
\forall \mathbf{x} \in \Omega \quad, \quad E\left\{\left\|\left[\mathbf{A}_{n}(\mathbf{x})\right]\right\|^{2}\right\} \leq E\left\{\left\|\left[\mathbf{A}_{n}(\mathbf{x})\right]\right\|_{F}^{2}\right\} \leq n \underline{c}_{1}^{2} E\left\{\left\|\left[\mathbf{G}_{n}(\mathbf{x})\right]\right\|_{F}^{2}\right\}<+\infty \quad,
$$

which proves that $\mathbf{x} \mapsto\left[\mathbf{A}_{n}(\mathbf{x})\right]$ is a second-order random field on $\Omega$.

(iii) Homogeneousness. In general, since $\left[\underline{a}_{n}(\mathbf{x})\right]$ depends on $\mathbf{x}$, random field $\left\{\left[\mathbf{A}_{n}(\mathbf{x})\right], \mathbf{x} \in \Omega\right\}$ is non homogeneous. Nevertheless, if $\Omega=\mathbb{R}^{d}$ and if $\left[\underline{a}_{n}(\mathbf{x})\right]=\left[\underline{a}_{n}\right]$ is independent of $\mathbf{x}$, then the random field $\left\{\left[\mathbf{A}_{n}(\mathbf{x})\right]=\left[\underline{L}_{n}\right]^{T}\left[\mathbf{G}_{n}(\mathbf{x})\right]\left[\underline{L}_{n}\right], \mathbf{x} \in \Omega\right\}$ can be viewed as the restriction to $\Omega$ of an homogeneous random field indexed by $\mathbb{R}^{d}$.

(iv) Mean-function. From Eqs. (36), (71), (72) and (77), we deduce that the mean function $\mathbf{x} \mapsto E\left\{\left[\mathbf{A}_{n}(\mathbf{x})\right]\right\}$ from $\Omega$ into $\mathbb{M}_{n}^{+}(\mathbb{R})$ is such that

$$
\forall \mathbf{x} \in \Omega \quad, \quad E\left\{\left[\mathbf{A}_{n}(\mathbf{x})\right]\right\}=\left[\underline{a}_{n}(\mathbf{x})\right] \in \mathbb{M}_{n}^{+}(\mathbb{R})
$$

(v) Fourth-order tensor-valued covariance function. This is the function $\left(\mathbf{x}, \mathbf{x}^{\prime}\right) \mapsto C^{A_{n}}\left(\mathbf{x}, \mathbf{x}^{\prime}\right)$ defined on $\Omega \times \Omega$ such that

$$
C^{A_{n}}\left(\mathbf{x}, \mathbf{x}^{\prime}\right)=E\left\{\left(\left[\mathbf{A}_{n}(\mathbf{x})\right]-\left[\underline{a}_{n}(\mathbf{x})\right]\right) \otimes\left(\left[\mathbf{A}_{n}\left(\mathbf{x}^{\prime}\right)\right]-\left[\underline{a}_{n}\left(\mathbf{x}^{\prime}\right)\right]\right)\right\} \quad .
$$

The covariance tensor $C^{A_{n}}(\mathbf{x}, \mathbf{x})$ of random matrix $\left[\mathbf{A}_{n}(\mathbf{x})\right]$ which is such that

$$
C_{j k, j^{\prime} k^{\prime}}^{A_{n}}(\mathbf{x}, \mathbf{x})=E\left\{\left(\left[\mathbf{A}_{n}(\mathbf{x})\right]_{j k}-\left[\underline{a}_{n}(\mathbf{x})\right]_{j k}\right)\left(\left[\mathbf{A}_{n}(\mathbf{x})\right]_{j^{\prime} k^{\prime}}-\left[\underline{a}_{n}(\mathbf{x})\right]_{j^{\prime} k^{\prime}}\right)\right\}
$$

is then given (see Refs. [74,75]) by

$$
C_{j k, j^{\prime} k^{\prime}}^{A_{n}}(\mathbf{x}, \mathbf{x})=\frac{\delta^{2}}{n+1}\left\{\left[\underline{a}_{n}(\mathbf{x})\right]_{j^{\prime} k}\left[\underline{a}_{n}(\mathbf{x})\right]_{j k^{\prime}}+\left[\underline{a}_{n}(\mathbf{x})\right]_{j j^{\prime}}\left[\underline{a}_{n}(\mathbf{x})\right]_{k k^{\prime}}\right\} .
$$

Consequently, the variance $V_{j k}^{A_{n}}(\mathbf{x})$ of random variable $\left[\mathbf{A}_{n}(\mathbf{x})\right]_{j k}$ can be written as

$$
V_{j k}^{A_{n}}(\mathbf{x})=\frac{\delta^{2}}{n+1}\left\{\left[\underline{a}_{n}(\mathbf{x})\right]_{j k}^{2}+\left[\underline{a}_{n}(\mathbf{x})\right]_{j j}\left[\underline{a}_{n}(\mathbf{x})\right]_{k k}\right\} \quad .
$$

Since $E\left\{\left\|\left[\mathbf{A}_{n}(\mathbf{x})\right]-\left[\underline{a}_{n}(\mathbf{x})\right]\right\|_{F}^{2}\right\}=\sum_{j, k=1}^{n} V_{j k}^{A_{n}}(\mathbf{x})$, we then have

$$
E\left\{\left\|\left[\mathbf{A}_{n}(\mathbf{x})\right]-\left[\underline{a}_{n}(\mathbf{x})\right]\right\|_{F}^{2}\right\}=\frac{\delta^{2}}{n+1}\left\{\left\|\left[\underline{a}_{n}(\mathbf{x})\right]\right\|_{F}^{2}+\left(\operatorname{tr}\left[\underline{a}_{n}(\mathbf{x})\right]\right)^{2}\right\} .
$$


(vi) Dispersion parameter. The dispersion of random field $\mathbf{x} \mapsto\left[\mathbf{A}_{n}(\mathbf{x})\right]$ indexed by $\Omega$ is controlled by parameter $\delta$ defined by Eq. (37) and is independent of $\mathbf{x}$ and $n$. Let $\delta_{A_{n}}(\mathbf{x})$ be the parameter defined by

$$
\delta_{A_{n}}(\mathbf{x})=\left\{\frac{E\left\{\left\|\left[\mathbf{A}_{n}(\mathbf{x})\right]-\left[\underline{a}_{n}(\mathbf{x})\right]\right\|_{F}^{2}\right\}}{\left\|\left[\underline{a}_{n}(\mathbf{x})\right]\right\|_{F}^{2}}\right\}^{1 / 2} .
$$

From Eqs. (86) and (87), we deduce that

$$
\delta_{A_{n}}(\mathbf{x})=\frac{\delta}{\sqrt{n+1}}\left\{1+\frac{\left(\operatorname{tr}\left[\underline{a}_{n}(\mathbf{x})\right]\right)^{2}}{\operatorname{tr}\left\{\left[\underline{a}_{n}(\mathbf{x})\right]^{2}\right\}}\right\}^{1 / 2} .
$$

Equation (88) shows that $\delta_{A_{n}}(\mathbf{x})$ depends on $\mathbf{x}, n$ and $\delta$.

(vii) Numerical simulation of the random field. The numerical simulation of the random field $\mathbf{x} \mapsto\left[\mathbf{A}_{n}(\mathbf{x})\right]$ can easily be constructed. Let $\mathbf{x} \mapsto\left[\mathbf{A}_{n}(\mathbf{x}, \theta)\right]$ be such a realization for $\theta \in \Theta$. Equation (77) allows this realization to be constructed as

$$
\left[\mathbf{A}_{n}(\mathbf{x}, \theta)\right]=\left[\underline{L}_{n}(\mathbf{x})\right]^{T}\left[\mathbf{G}_{n}(\mathbf{x}, \theta)\right]\left[\underline{L}_{n}(\mathbf{x})\right],
$$

in which the realization $\mathbf{x} \mapsto\left[\mathbf{G}_{n}(\mathbf{x}, \theta)\right]$ of the random field $\mathbf{x} \mapsto\left[\mathbf{G}_{n}(\mathbf{x})\right]$ is calculated by using Eq. (44), i.e.,

$$
\forall \mathbf{x} \in \Omega \quad, \quad\left[\mathbf{G}_{n}(\mathbf{x}, \theta)\right]=\left[g_{n}(\mathbb{U}(\mathbf{x}, \theta))\right] .
$$

The realization $\mathbf{x} \mapsto \mathbb{U}(\mathbf{x}, \theta)$ of the Gaussian random field $\mathbf{x} \mapsto \mathbb{U}(\mathbf{x})=\left\{U_{j j^{\prime}}(\mathbf{x}), 1 \leq j \leq j^{\prime} \leq n\right\}$ is constructed by using Section 3.2.3.

(viii) Invertibility. From Eqs. (46), (74) and (77), it can be deduced that there exists a finite positive constant $c_{n}$ dependent on $n$ and $\delta$ but independent of $\mathbf{x}$, such that

$$
E\left\{\left\|\left[\mathbf{A}_{n}(\mathbf{x})\right]^{-1}\right\|^{2}\right\} \leq c_{n}<+\infty \quad, \quad \forall \mathbf{x} \in \mathbb{R}^{d}
$$

\subsection{Spatial correlation lengths of the random field $\mathbf{x} \mapsto\left[\mathbf{A}_{n}(\mathbf{x})\right]$ for the homogeneous case}

In this section, it is assumed that $\left[\underline{a}_{n}(\mathbf{x})\right]=\left[\underline{a}_{n}\right]$ is independent of $\mathbf{x}$. From Eq. (77), it can be deduced that the random field $\mathbf{x} \mapsto\left[\mathbf{A}_{n}(\mathbf{x})\right]$ is such that, for all $\mathbf{x} \in \Omega$,

$$
\left[\mathbf{A}_{n}(\mathbf{x})\right]=\left[\underline{L}_{n}\right]^{T}\left[\mathbf{G}_{n}(\mathbf{x})\right]\left[\underline{L}_{n}\right],
$$


in which $\left[\underline{L}_{n}\right]$ is the upper triangular $(n \times n)$ real matrix deduced from the Chowlesky factorization of the positive-definite symmetric $(n \times n)$ real matrix $\left[\underline{a}_{n}\right]$ such that

$$
\left[\underline{a}_{n}\right]=\left[\underline{L}_{n}\right]^{T}\left[\underline{L}_{n}\right]
$$

Consequently, the random field $\mathbf{x} \mapsto\left[\mathbf{A}_{n}(\mathbf{x})\right]$ in homogeneous on $\mathbb{R}^{d}$. In this case, from Eq. (88), it can be deduced that $\delta_{A_{n}}(\mathbf{x})=\delta_{A_{n}}$ is independent of $\mathbf{x}$ and is written as

$$
\delta_{A_{n}}=\frac{\delta}{\sqrt{n+1}}\left\{1+\frac{\left(\operatorname{tr}\left[\underline{a}_{n}\right]\right)^{2}}{\operatorname{tr}\left\{\left[\underline{a}_{n}\right]^{2}\right\}}\right\}^{1 / 2}
$$

Let $\boldsymbol{\eta}=\left(\eta_{1}, \ldots, \eta_{d}\right) \mapsto r^{A_{n}}(\boldsymbol{\eta})$ be the function defined from $\mathbb{R}^{d}$ into $\mathbb{R}$ by

$$
r^{A_{n}}(\boldsymbol{\eta})=\frac{\operatorname{tr} E\left\{\left(\left[\mathbf{A}_{n}(\mathbf{x}+\boldsymbol{\eta})\right]-\left[\underline{a}_{n}\right]\right)\left(\left[\mathbf{A}_{n}(\mathbf{x})\right]-\left[\underline{a}_{n}\right]\right)\right\}}{E\left\{\left\|\left[\mathbf{A}_{n}(\mathbf{x})\right]-\left[\underline{a}_{n}\right]\right\|_{F}^{2}\right\}} .
$$

From Eq. (87), it is deduced that

$$
\begin{gathered}
r^{A_{n}}(\boldsymbol{\eta})=\frac{E\left\{\operatorname{tr}\left\{\left[\mathbf{A}_{n}(\mathbf{x}+\boldsymbol{\eta})\right]\left[\mathbf{A}_{n}(\mathbf{x})\right]\right\}\right\}-\operatorname{tr}\left\{\left[\underline{a}_{n}\right]^{2}\right\}}{\delta_{A_{n}}^{2} \operatorname{tr}\left\{\left[\underline{a}_{n}\right]^{2}\right\}}, \\
r^{A_{n}}(0)=1 \quad, \quad r^{A_{n}}(-\boldsymbol{\eta})=r^{A_{n}}(\boldsymbol{\eta}) .
\end{gathered}
$$

For all $j=1, \ldots, d$, the spatial correlation length $L_{j}^{A_{n}}$ of the homogeneous random field $\mathbf{x} \mapsto$ $\left[\mathbf{A}_{n}(\mathbf{x})\right]$ indexed by $\mathbb{R}^{d}$, relative to coordinate $x_{j}$, can be defined by

$$
L_{j}^{A_{n}}=\int_{0}^{+\infty}\left|r^{A_{n}}\left(0, \ldots, 0, \eta_{j}, 0, \ldots, 0\right)\right| d \eta_{j}
$$

\section{Elliptic stochastic partial differential operator}

In this section, we consider the second-order stochastic differential operator defined by Eq. (4), associated with the mean elliptic partial differential operator defined by Eq. (1) in the context of 3D linear elasticity. All the results presented below can straightforwardly be extended to a general elliptic partial differential operator [58,88].

C. Soize - CMAME - revised version December 2004 


\subsection{Mean elliptic boundary value problem (BVP)}

Let $\Omega$ be an open bounded domain of $\mathbb{R}^{d}$ with $d=3$. Let $\partial \Omega=\Gamma_{0} \cup \Gamma$ be the boundary of $\Omega$ which is assumed to be sufficiently smooth. Let $\boldsymbol{\nu}=\left(\nu_{1}, \nu_{2}, \nu_{3}\right)$ be the unit normal to $\partial \Omega$ external to $\Omega$ (see Fig. 4).

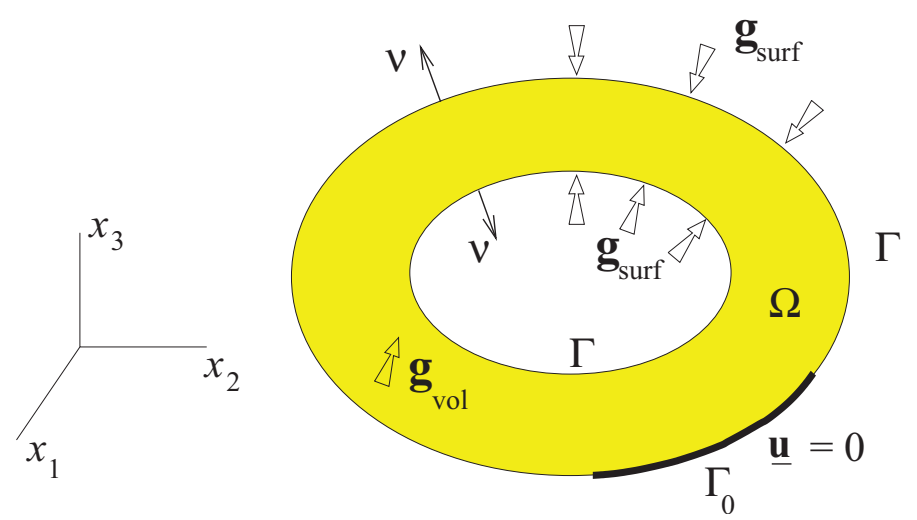

Fig. 4. Geometrical configuration

We consider the following mean elliptic boundary value problem (BVP): Find a function $\mathbf{x} \mapsto$ $\underline{\mathbf{v}}(\mathbf{x})=\left(\underline{v}_{1}(\mathbf{x}), \underline{v}_{2}(\mathbf{x}), \underline{v}_{3}(\mathbf{x})\right)$ from $\Omega$ into $\mathbb{R}^{3}$ such that

$$
\begin{aligned}
-\operatorname{div} \underline{\boldsymbol{\sigma}} & =\mathbf{g}_{\mathrm{vol}} \quad, \quad \text { in } \quad \Omega, \\
\underline{\mathbf{v}}(\mathbf{x}) & =0 \quad, \quad \text { on } \quad \Gamma_{0}, \\
\underline{\boldsymbol{\sigma}} \boldsymbol{\nu} & =\mathbf{g}_{\text {surf }} \quad, \quad \text { on } \Gamma .
\end{aligned}
$$

For a non homogeneous anisotropic elastic material occupying domain $\Omega$, the stress tensor $\underline{\boldsymbol{\sigma}}=$ $\left\{\underline{\sigma}_{i j}\right\}_{i j}$ and the strain tensor $\varepsilon=\left\{\varepsilon_{k h}\right\}_{k h}$ defined by

$$
\varepsilon_{k h}(\underline{\mathbf{v}})=\frac{1}{2}\left(\frac{\partial \underline{v}_{k}}{\partial x_{h}}+\frac{\partial \underline{v}_{h}}{\partial x_{k}}\right)
$$

are such that (constitutive equation),

$$
\underline{\sigma}_{i j}=\sum_{k, h=1}^{3} \underline{c}_{i j k h}(\mathbf{x}) \varepsilon_{k h}(\underline{\mathbf{v}}) .
$$

In Eq. (98), $\left.\mathbf{x} \mapsto \mathbf{g}_{\mathrm{vol}}(\mathbf{x})\right)$ is assumed to be a given square integrable function (for the Lebesgue measure $d \mathbf{x})$ from $\Omega$ into $\mathbb{R}^{3}$ and $\{\operatorname{div} \underline{\boldsymbol{\sigma}}\}_{i}=\sum_{j=1}^{3} \partial \underline{\sigma}_{i j} / \partial x_{j}$. In Eq. (100), $\left.\mathbf{x} \mapsto \mathbf{g}_{\text {surf }}(\mathbf{x})\right)$ is assumed to be a given square integrable function (for the surface measure $d s$ ) from $\Gamma$ into $\mathbb{R}^{3}$ and $\{\underline{\boldsymbol{\sigma}} \boldsymbol{\nu}\}_{i}=\sum_{j=1}^{3} \underline{\sigma}_{i j} \nu_{j}$. The fourth-order tensor $\left\{\underline{c}_{i j k h}(\mathbf{x})\right\}_{i j k h}$ of the elastic coefficients verifies the properties of symmetry and positiveness defined by Eqs. (2) and (3) respectively. In addition, it is assumed that $\mathbf{x} \mapsto \underline{c}_{i j k h}(\mathbf{x})$ are bounded functions on $\Omega$. 


\subsection{Weak formulation of the mean BVP}

We introduce the real Hilbert space $\mathcal{H}=\left\{\mathbf{v}=\left(v_{1}, v_{2}, v_{3}\right), v_{j} \in L^{2}(\Omega)\right\}$ equipped with the inner product

$$
<\mathbf{v}, \mathbf{w}>_{\mathcal{H}}=\int_{\Omega}<\mathbf{v}(\mathbf{x}), \mathbf{w}(\mathbf{x})>d \mathbf{x}
$$

and with the associated norm $\|\mathbf{v}\|_{\mathcal{H}}=\left\langle\mathbf{v}, \mathbf{v}>_{\mathcal{H}}^{1 / 2}\right.$ where $L^{2}(\Omega)$ denotes the set of all the square integrable functions from $\Omega$ into $\mathbb{R}$. Let $\mathcal{V} \subset \mathcal{H}$ be the real Hilbert space representing the set of admissible displacement fields with values in $\mathbb{R}^{3}$ such that

$$
\mathcal{V}=\left\{\mathbf{v} \in \mathcal{H} \quad, \quad \partial \mathbf{v} / \partial x_{j} \in \mathcal{H} \quad, \quad \mathbf{v}=0 \quad \text { on } \quad \Gamma_{0}\right\}
$$

equipped with the inner product

$$
<\mathbf{v}, \mathbf{w}>_{\mathcal{V}}=<\mathbf{v}, \mathbf{w}>_{\mathcal{H}}+\sum_{j=1}^{3}<\frac{\partial \mathbf{v}}{\partial x_{j}}, \frac{\partial \mathbf{w}}{\partial x_{j}}>_{\mathcal{H}}
$$

and with the associated norm $\|\mathbf{v}\|_{\mathcal{V}}=<\mathbf{v}, \mathbf{v}>_{\mathcal{V}}^{1 / 2}$. The usual weak formulation of the mean boundary value problem defined by Eqs. (98) to (102) is the following (see for instance Ref. [10,49]): find the function $\{\mathbf{x} \mapsto \underline{\mathbf{v}}(\mathbf{x})\}$ in $\mathcal{V}$ such that

$$
\underline{k}(\underline{\mathbf{v}}, \mathbf{w})=f(\mathbf{w}) \quad, \quad \forall \mathbf{w} \in \mathcal{V} \quad
$$

(i)The linear form $\mathbf{w} \mapsto f(\mathbf{w})$ on $\mathcal{V}$ is defined by

$$
f(\mathbf{w})=\int_{\Omega}<\mathbf{g}_{\mathrm{vol}}(\mathbf{x}), \mathbf{w}(\mathbf{x})>d \mathbf{x}+\int_{\Gamma}<\mathbf{g}_{\mathrm{surf}}(\mathbf{x}), \mathbf{w}(\mathbf{x})>d s(\mathbf{x})
$$

is continuous on $\mathcal{V}$ :

$$
|f(\mathbf{w})| \leq c_{f}\|\mathbf{w}\|_{\mathcal{V}} \quad, \quad 0<c_{f}<+\infty \quad
$$

(ii) Thesymmetric bilinear form $(\mathbf{v}, \mathbf{w}) \mapsto \underline{k}(\mathbf{v}, \mathbf{w})$ on $\mathcal{V} \times \mathcal{V}$, defined by

$$
\underline{k}(\mathbf{v}, \mathbf{w})=\int_{\Omega} \sum_{i, j, k, h=1}^{3} \underline{c}_{i j k h}(\mathbf{x}) \varepsilon_{k h}(\mathbf{v}) \varepsilon_{i j}(\mathbf{w}) d \mathbf{x}
$$

is continuous on $\mathcal{V} \times \mathcal{V}$ (due to the boundness of coefficients $\underline{c}_{i j k h}(\mathbf{x})$ on $\Omega$ ) and is $\mathcal{V}$-elliptic (due to Eq. (3)), that is to say

$$
\underline{k}(\mathbf{v}, \mathbf{v}) \geq \underline{c}_{1}\|\mathbf{v}\|_{\mathcal{V}}^{2} \quad, \quad 0<\underline{c}_{1}<+\infty
$$

Consequently, due to the Lax-Milgram theorem, there is a unique solution $\underline{\mathbf{v}} \in \mathcal{V}$ of Eq. (106) which is the unique weak solution of Eqs. (98)-(102). 


\subsection{Rewritting the bilinear form using matrix notation}

Let us introduce the new indices $I$ and $J$ belonging to $\{1, \ldots, 6\}$ such that $I=(i, j)$ and $J=(k, h)$ with the following correspondence: $1=(1,1), 2=(2,2), 3=(3,3), 4=(1,2), 5=(2,3)$ and $6=(3,1)$. Consequently, the bilinear form $\underline{k}(\mathbf{v}, \mathbf{w})$ defined by Eq. (109) can be rewritten as

$$
\underline{k}(\mathbf{v}, \mathbf{w})=\int_{\Omega}<\left[\underline{a}_{n}(\mathbf{x})\right] \mathbf{e}(\mathbf{v}(\mathbf{x})), \mathbf{e}(\mathbf{w}(\mathbf{x}))>d \mathbf{x} \quad,
$$

in which $\left[\underline{a}_{n}(\mathbf{x})\right]$ is the real $(n \times n)$ matrix (with $\left.n=6\right)$ such that $\left[\underline{a}_{n}(\mathbf{x})\right]_{I J}=\underline{c}_{i j k h}(\mathbf{x})$ and where $\mathbf{e}(\mathbf{v})=\left(e_{1}(\mathbf{v}), \ldots, e_{n}(\mathbf{v})\right)$ with $e_{1}(\mathbf{v})=\varepsilon_{11}(\mathbf{v}), e_{2}(\mathbf{v})=\varepsilon_{22}(\mathbf{v}), e_{3}(\mathbf{v})=\varepsilon_{33}(\mathbf{v}), e_{4}(\mathbf{v})=2 \varepsilon_{12}(\mathbf{v})$, $e_{5}(\mathbf{v})=2 \varepsilon_{23}(\mathbf{v})$ and $e_{6}(\mathbf{v})=2 \varepsilon_{31}(\mathbf{v})$. From the properties of symmetry, positiveness and boundedness of tensor $\underline{c}_{i j k h}(\mathbf{x})$, it can directly be deduced that $\mathbf{x} \mapsto\left[\underline{a}_{n}(\mathbf{x})\right]$ is a matrix-valued field from $\Omega$ into $\mathbb{M}_{n}^{+}(\mathbb{R})$, bounded on $\Omega$, which satisfies all the properties defined by Eqs. (71) to (76).

\subsection{Nonparametric probabilistic modeling of the elasticity tensor}

Since matrix field $\mathbf{x} \mapsto\left[\underline{a}_{n}(\mathbf{x})\right]$ from $\Omega$ into $\mathbb{M}_{n}^{+}(\mathbb{R})$ verifies Eqs. (71) to (76), the random non homogeneous anisotropic elastic material can be constructed in substituting in the constitutive equation the field $\mathbf{x} \mapsto\left[\underline{a}_{n}(\mathbf{x})\right]$ by a random field $\mathbf{x} \mapsto\left[\mathbf{A}_{n}(\mathbf{x})\right]$ indexed by $\Omega$ with values in $M_{n}^{+}(\mathbb{R})$, belonging to the ensemble SFE ${ }^{+}$. This random field is thus defined by Eq. (77) in which the stochastic field $\mathbf{x} \mapsto\left[\mathbf{G}_{n}(\mathbf{x})\right]$ belonging to the ensemble $\mathrm{SFG}^{+}$is defined in Section 3, where $E\left\{\left[\mathbf{A}_{n}(\mathbf{x})\right]\right\}=\left[\underline{a}_{n}(\mathbf{x})\right]$ and where $\left[\underline{L}_{n}(\mathbf{x})\right]$ is defined by Eq. (72). Therefore, the bilinear form $\underline{k}(\mathbf{v}, \mathbf{w})$ defined by Eq. (111) is replaced by the random bilinear form $\mathbf{K}(\mathbf{v}, \mathbf{w})$ such that

$$
\mathbf{K}(\mathbf{v}, \mathbf{w})=\int_{\Omega}<\left[\mathbf{A}_{n}(\mathbf{x})\right] \mathbf{e}(\mathbf{v}(\mathbf{x})), \mathbf{e}(\mathbf{w}(\mathbf{x}))>d \mathbf{x}
$$

\subsection{Weak formulation of the stochastic BVP}

Let $\mathbb{H}=L^{2}(\Theta, \mathcal{H})$ (or $\mathbb{V}=L^{2}(\Theta, \mathcal{V})$ ) be the real Hilbert space of the second-order random variable $\theta \mapsto\{\mathbf{x} \mapsto \mathbf{V}(\mathbf{x}, \theta)\}$ defined on probability space $(\Theta, \mathcal{T}, P)$ with values in $\mathcal{H}$ (or in $\mathcal{V}$ ), equipped with the inner product

$$
\left.\ll \mathbf{V}, \mathbf{W} \gg_{\mathbb{H}}=E\{<\mathbf{V}, \mathbf{W}\rangle_{\mathcal{H}}\right\} \quad, \quad\left(\text { or } \quad \ll \mathbf{V}, \mathbf{W} \gg_{\mathbb{V}}=E\{<\mathbf{V}, \mathbf{W}\rangle_{\mathcal{V}}\right\} \quad,
$$

and with the associated norm $\|\mathbf{V}\|_{\mathbb{H}}=\ll \mathbf{V}, \mathbf{V} \gg_{\mathbb{H}}^{1 / 2}$ (or $\|\mathbf{V}\|_{V}=\ll \mathbf{V}, \mathbf{V} \gg_{V}^{1 / 2}$ ). Using the stochastic modeling defined in Section 5.4, the mean problem defined by Eq. (106) is replaced by the following random problem: Find the random field $\{\mathbf{x} \mapsto \mathbf{V}(\mathbf{x})\}$ in $\mathbb{V}$ such that

$$
\mathbf{K}(\mathbf{V}, \mathbf{W})=f(\mathbf{W}) \quad \text { a.s. } \quad, \quad \forall \mathbf{W} \in \mathbb{V},
$$


in which the random bilinear from $\mathbf{K}$ is defined by Eq. (112). Taking the mathematical expectation of the two members of Eq. (113), the weak formulation of the stochastic BVP is then the following: Find the random field $\{\mathbf{x} \mapsto \mathbf{V}(\mathbf{x})\}$ in $\mathbb{V}$ such that

$$
\mathcal{K}(\mathbf{V}, \mathbf{W})=E\{f(\mathbf{W})\} \quad, \quad \forall \mathbf{W} \in \mathbb{V},
$$

in which the symmetric bilinear form $(\mathbf{V}, \mathbf{W}) \mapsto \mathcal{K}(\mathbf{V}, \mathbf{W})$ on $\mathbb{V} \times \mathbb{V}$ is defined by

$$
\begin{aligned}
\mathcal{K}(\mathbf{V}, \mathbf{W}) & =E\{\mathbf{K}(\mathbf{V}, \mathbf{W})\} \\
& =E\left\{\int_{\Omega}<\left[\mathbf{A}_{n}(\mathbf{x})\right] \mathbf{e}(\mathbf{V}(\mathbf{x})), \mathbf{e}(\mathbf{W}(\mathbf{x}))>d \mathbf{x}\right\} .
\end{aligned}
$$

Let us prove that the bilinear form $(\mathbf{V}, \mathbf{W}) \mapsto \mathcal{K}(\mathbf{V}, \mathbf{W})$ is continous on $\mathbb{V} \times \mathbb{V}$. From Eq. (35), it can be deduced that

$$
E\left\{\left\|\left[\mathbf{G}_{n}(\mathbf{x})\right]\right\|^{2}\right\} \leq E\left\{\left\|\left[\mathbf{G}_{n}(\mathbf{x})\right]\right\|_{F}^{2}\right\}=c<+\infty \quad, \quad \forall \mathbf{x} \in \mathbb{R}^{d} .
$$

From Eqs. (77), (111) and (115), we have

$$
\begin{aligned}
|\mathcal{K}(\mathbf{V}, \mathbf{W})| & \leq E\left\{\int_{\Omega}\left\|\left[\mathbf{G}_{n}(\mathbf{x})\right]\right\|\left\|\left[\underline{L}_{n}(\mathbf{x})\right] \mathbf{e}(\mathbf{V}(\mathbf{x}))\right\|\left\|\left[\underline{L}_{n}(\mathbf{x})\right] \mathbf{e}(\mathbf{W}(\mathbf{x}))\right\| d \mathbf{x}\right\} \\
& \leq\left(\int_{\Omega} E\left\{\left\|\left[\mathbf{G}_{n}(\mathbf{x})\right]\right\|^{2}\right\} d \mathbf{x}\right)^{1 / 2}(E\{\underline{k}(\mathbf{V}, \mathbf{V})\} E\{\underline{k}(\mathbf{W}, \mathbf{W})\})^{1 / 2} .
\end{aligned}
$$

Since the bilinear form $(\mathbf{v}, \mathbf{w}) \mapsto \underline{k}(\mathbf{v}, \mathbf{w})$ is continuous on $\mathcal{V} \times \mathcal{V}$ (see Section 5.2.ii) and since $\Omega$ is bounded, we deduce that

$$
|\mathcal{K}(\mathbf{V}, \mathbf{W})| \leq c\|\mathbf{V}\|_{\mathbb{V}}\|\mathbf{W}\|_{\mathbb{V}}
$$

in which $c$ is a deterministic finite real constant.

5.6. Why the exitence of a solution for the weak formulation of the stochastic BVP cannot be simply studied

(i) If the following property is introduced (for instance, as introduced in Ref. [6]): for all $\mathbf{x} \in \Omega$ and for all $\mathbb{R}^{n}$-valued random variable $\mathbf{Y}$ defined on $(\Theta, \mathcal{T}, P)$,

$$
<\left[\mathbf{A}_{n}(\mathbf{x})\right] \mathbf{Y}, \mathbf{Y}>\geq \underline{c}_{0}\|\mathbf{Y}\|^{2} \text { a.s }
$$

in which $0<\underline{c}_{0}<+\infty$ is independent of $\mathbf{x}$, then the bilinear form $(\mathbf{V}, \mathbf{W}) \mapsto \mathcal{K}(\mathbf{V}, \mathbf{W})$ on $\mathbb{V} \times \mathbb{V}$ is coercive in $\mathbb{V}$ (i.e. $\mathbb{V}$-elliptic) because

$$
\mathcal{K}(\mathbf{V}, \mathbf{V}) \geq \underline{c}_{0} E\left\{\int_{\Omega}\|\mathbf{e}(\mathbf{V}(\mathbf{x}))\|^{2} d \mathbf{x}\right\} \geq c_{\mathcal{K}}\|\mathbf{V}\|_{\mathbb{V}}^{2} \quad, \quad 0<c_{\mathcal{K}}<+\infty
$$

Taking into account Eqs. (108) and (117), in view of the Lax-Milgram theorem [10], the problem defined by Eq. (114) has a unique solution $\mathbf{V}$ in $\mathbb{V}$. 
(ii) From Sections 3 and 4, it can be seen that the property defined by Eq. (116) does not hold for the random field $\mathbf{x} \mapsto\left[\mathbf{A}_{n}(\mathbf{x})\right]$ belonging to $\mathrm{SFE}^{+}$and consequently, the usual analysis given in paragraph (i) above cannot presently be used. Another analysis has to be developed using the fundamental property defined by Eq. (56) and which constitutes the mathematical property replacing Eq. (116).

(iii) It can be interesting to explain why such a property defined by Eq. (116) has not been introduced in Sections 3 and 4 for the construction of the ensemble $\mathrm{SFE}^{+}$of the random fields $\mathbf{x} \mapsto\left[\mathbf{A}_{n}(\mathbf{x})\right]$. From the construction of the ensemble $\mathrm{SFE}^{+}$(see Eqs. (78), (80), (81) and (90)), we have deduced that, for all $\mathbf{x}$ fixed in $\Omega$, we have the following properties.

(C1) $\left[\mathbf{A}_{n}(\mathbf{x})\right]$ is a second-order random matrix with values in $M_{n}^{+}(\mathbb{R})$.

(C2) The mean value $E\left\{\left[\mathbf{A}_{n}(\mathbf{x})\right]\right\}$ is a matrix $\left[\underline{a}_{n}(\mathbf{x})\right]$ given in $\mathbb{M}_{n}^{+}(\mathbb{R})$.

(C3) We have $E\left\{\left\|\left[\mathbf{A}_{n}(\mathbf{x})\right]^{-1}\right\|^{2}\right\} \leq c_{n}<+\infty$ for all $\mathbf{x}$ fixed in $\Omega$.

In addition, for all $\mathbf{x}$ fixed in $\Omega$, the probability distribution $P_{\left[\mathbf{A}_{n}(\mathbf{x})\right]}$ on $\mathbb{M}_{n}^{+}(\mathbb{R})$ of the random matrix $\left[\mathbf{A}_{n}(\mathbf{x})\right]$ can be written as $P_{\left[\mathbf{A}_{n}(\mathbf{x})\right]}=p_{\left[\mathbf{A}_{n}(\mathbf{x})\right]}\left(\left[A_{n}\right], \mathbf{x}\right) \widetilde{d} A_{n}$ in which the probability density function $\left[A_{n}\right] \mapsto p_{\left[\mathbf{A}_{n}(\mathbf{x})\right]}\left(\left[A_{n}\right], \mathbf{x}\right)$ from $\mathbb{M}_{n}^{+}(\mathbb{R})$ into $\mathbb{R}^{+}$, with respect to the measure $\widetilde{d} A_{n}=2^{n(n-1) / 4} \Pi_{1 \leq i \leq j \leq n} d\left[A_{n}\right]_{i j}$, can easily be calculated from Eqs. (42) and (77) (see the explicit expression of this probability density function in Refs. [74,75]). However, in Refs.[74,75], it is proved that this probability density function $\left[A_{n}\right] \mapsto p_{\left[\mathbf{A}_{n}(\mathbf{x})\right]}\left(\left[A_{n}\right], \mathbf{x}\right)$ corresponds to the unique probability density function constructed with the maximum entropy principle for which the constraints are the available information defined by $(\mathrm{C} 1),(\mathrm{C} 2)$ and $(\mathrm{C} 3)$ above. The constraint $(\mathrm{C} 3)$ allows the ellipticity condition defined by Eq. (118) to be obtained but does not allow the ellipticity condition defined by Eq. (117) to be obtained, this last ellipticity condition being directly deduced from Eq. (116). Equation (116) would be verified if constraint (C3) was replaced by a constraint (C3') related to the support of the probability density function $\left[A_{n}\right] \mapsto p_{\left[\mathbf{A}_{n}(\mathbf{x})\right]}\left(\left[A_{n}\right], \mathbf{x}\right)$ for that Eq. (116) be verified. In this paper, we are interested in the realistic situations for which such a constraint (C3') is considered as a non available information, that is to say corresponds to a non objective data. This is the case if the property defined by (C3') and the lower bound $\underline{c}_{0}$ in Eq. (116) cannot be tested and estimated by using mathematical statistics applied to experimental data.

\subsection{Ellipticity of the random bilinear form}

Let $\mathbf{K}(\mathbf{v}, \mathbf{w})$ be the random bilinear form defined by Eq. (112) in which the random field $\mathbf{x} \mapsto\left[\mathbf{A}_{n}(\mathbf{x})\right]$ 
is defined in Section 5.4. Then for all random field $\{\mathbf{x} \mapsto \mathbf{V}(\mathbf{x})\}$ in $\mathbb{V}$, we have

$$
\sqrt{E\left\{\mathbf{K}(\mathbf{V}, \mathbf{V})^{2}\right\}} \geq c_{K}\|\mathbf{V}\|_{\mathbb{V}}^{2}
$$

in which $c_{K}$ is a positive finite real constant.

Proof. From Eqs. (110) and (111), it can be deduced that, for all random field $\{\mathbf{x} \mapsto \mathbf{V}(\mathbf{x})\}$ in $\mathbb{V}, \underline{c}_{1}\|\mathbf{V}\|_{\mathcal{V}}^{2} \leq \underline{k}(\mathbf{V}, \mathbf{V})=\int_{\Omega}<\left[\underline{a}_{n}(\mathbf{x})\right] \mathbf{e}(\mathbf{V}(\mathbf{x})), \mathbf{e}(\mathbf{V}(\mathbf{x}))>d \mathbf{x}$. Since $\left[\mathbf{G}_{n}(\mathbf{x})\right]$ is with values in $\mathbb{M}_{n}^{+}(\mathbb{R})$, we can write $\left[I_{n}\right]=\left[\mathbf{G}_{n}(\mathbf{x})\right]^{-1}\left[\mathbf{G}_{n}(\mathbf{x})\right]=\left[\mathbf{G}_{n}(\mathbf{x})\right]^{1 / 2}\left[\mathbf{G}_{n}(\mathbf{x})\right]^{-1}\left[\mathbf{G}_{n}(\mathbf{x})\right]^{1 / 2}$ and using Eq. (72) yields $\underline{c}_{1}\|\mathbf{V}\|_{\mathcal{V}}^{2} \leq \int_{\Omega}<\left[\mathbf{G}_{n}(\mathbf{x})\right]^{-1} \mathbf{Y}(\mathbf{x}), \mathbf{Y}(\mathbf{x})>d \mathbf{x}$, in which $\mathbf{Y}(\mathbf{x})=$ $\left[\mathbf{G}_{n}(\mathbf{x})\right]^{1 / 2}\left[\underline{L}_{n}(\mathbf{x})\right] \mathbf{e}(\mathbf{V}(\mathbf{x}))$. Thus, we have $<\left[\mathbf{G}_{n}(\mathbf{x})\right]^{-1} \mathbf{Y}(\mathbf{x}), \mathbf{Y}(\mathbf{x})>\leq\left\|\left[\mathbf{G}_{n}(\mathbf{x})\right]^{-1}\right\|\|\mathbf{Y}(\mathbf{x})\|^{2}$ and

$$
\begin{aligned}
\|\mathbf{Y}(\mathbf{x})\|^{2} & =<\left[\mathbf{G}_{n}(\mathbf{x})\right]^{1 / 2}\left[\underline{L}_{n}(\mathbf{x})\right] \mathbf{e}(\mathbf{V}(\mathbf{x})),\left[\mathbf{G}_{n}(\mathbf{x})\right]^{1 / 2}\left[\underline{L}_{n}(\mathbf{x})\right] \mathbf{e}(\mathbf{V}(\mathbf{x}))> \\
& =<\left[\underline{L}_{n}(\mathbf{x})\right]^{T}\left[\mathbf{G}_{n}(\mathbf{x})\right]\left[\underline{L}_{n}(\mathbf{x})\right] \mathbf{e}(\mathbf{V}(\mathbf{x})), \mathbf{e}(\mathbf{V}(\mathbf{x}))> \\
& =<\left[\mathbf{A}_{n}(\mathbf{x})\right] \mathbf{e}(\mathbf{V}(\mathbf{x})), \mathbf{e}(\mathbf{V}(\mathbf{x}))>,
\end{aligned}
$$

due to Eq. (77). Therefore,

$$
\begin{aligned}
\underline{c}_{1}\|\mathbf{V}\|_{\mathcal{V}}^{2} & \leq \int_{\Omega}\left\|\left[\mathbf{G}_{n}(\mathbf{x})\right]^{-1}\right\|<\left[\mathbf{A}_{n}(\mathbf{x})\right] \mathbf{e}(\mathbf{V}(\mathbf{x})), \mathbf{e}(\mathbf{V}(\mathbf{x}))>d \mathbf{x} \\
& \leq \sup _{\mathbf{x} \in \bar{\Omega}}\left\|\left[\mathbf{G}_{n}(\mathbf{x})\right]^{-1}\right\| \int_{\Omega}<\left[\mathbf{A}_{n}(\mathbf{x})\right] \mathbf{e}(\mathbf{V}(\mathbf{x})), \mathbf{e}(\mathbf{V}(\mathbf{x}))>d \mathbf{x} .
\end{aligned}
$$

From Eq. (112), this last inequality can be rewritten as

$$
\underline{c}_{1}\|\mathbf{V}\|_{\mathcal{V}}^{2} \leq\left(\sup _{\mathbf{x} \in \bar{\Omega}}\left\|\left[\mathbf{G}_{n}(\mathbf{x})\right]^{-1}\right\|\right) \mathbf{K}(\mathbf{V}, \mathbf{V})
$$

Since in Eq. (119), the three dependent random variables are with values in $\mathbb{R}^{+}$, we can take the mathematical expectation of the two members of the inequality. Since $E\left\{\|\mathbf{V}\|_{\mathcal{V}}^{2}\right\}=\|\mathbf{V}\|_{\mathcal{V}}^{2}$ and since $\left|E\left\{Z_{1} Z_{2}\right\}\right| \leq \sqrt{E\left\{Z_{1}^{2}\right\}} \sqrt{E\left\{Z_{2}^{2}\right\}}$ for two dependent real-valued random variables $Z_{1}$ and $Z_{2}$, we have

$$
\underline{c}_{1}\|\mathbf{V}\|_{\mathbb{V}}^{2} \leq \sqrt{E\left\{\left(\sup _{\mathbf{x} \in \bar{\Omega}}\left\|\left[\mathbf{G}_{n}(\mathbf{x})\right]^{-1}\right\|\right)^{2}\right\}} \sqrt{E\left\{\mathbf{K}(\mathbf{V}, \mathbf{V})^{2}\right\}} .
$$

Substituting Eq. (56) into Eq. (120) yields Eq. (118) with $c_{K}=\underline{c}_{1} / c_{G}$.

Remark. It should be noted that Eq. (120) differs from Eq. (117) which is written as $E\{\mathbf{K}(\mathbf{V}, \mathbf{V})\} \geq$ $c_{\mathcal{K}}\|\mathbf{V}\|_{\mathbb{V}}^{2}$ and not as $\sqrt{E\left\{\mathbf{K}(\mathbf{V}, \mathbf{V})^{2}\right\}} \geq c_{K}\|\mathbf{V}\|_{\mathbb{V}}^{2}$. This difference is due to the fact that the two positive-valued random variables $\sup _{\mathbf{x} \in \bar{\Omega}}\left\|\left[\mathbf{G}_{n}(\mathbf{x})\right]^{-1}\right\|$ and $\mathbf{K}(\mathbf{V}, \mathbf{V})$ are dependent. 


\subsection{Existence of a weak second-order stochastic solution of the stochastic BVP}

The random problem defined by Eq. (113) has a unique stochastic solution $\{\mathbf{x} \mapsto \mathbf{V}(\mathbf{x})\}$ in $\mathbb{V}$ which means that

$$
\|\mathbf{V}\|_{\mathbb{V}}^{2}=E\left\{\int_{\Omega}\left(\|\mathbf{V}(\mathbf{x})\|^{2}+\sum_{j=1}^{3}\left\|\frac{\partial \mathbf{V}(\mathbf{x})}{\partial x_{j}}\right\|^{2}\right) d \mathbf{x}\right\}<+\infty
$$

This equation yields

$$
E\left\{\int_{\Omega}\|\mathbf{V}(\mathbf{x})\|^{2} d \mathbf{x}\right\}<+\infty
$$

which shows that $\mathbf{x} \mapsto \mathbf{V}(\mathbf{x})$ is a second-order random field. In addition, the linear operator mapping the right-hand side $f$ onto the unique solution $\mathbf{V}$ is continuous from $\mathbb{V}^{\prime}$ to $\mathbb{V}$ in which $\mathbb{V}^{\prime}=L^{2}\left(\Theta, \mathcal{V}^{\prime}\right)$ where $\mathcal{V}^{\prime}$ is the continuous dual space of $\mathcal{V}$.

Proof. From Eqs. (108) and (113), we deduce that $\mathbf{K}(\mathbf{V}, \mathbf{V}) \leq c_{f}\|\mathbf{V}\|_{\mathcal{V}}$ and consequently, $E\left\{\mathbf{K}(\mathbf{V}, \mathbf{V})^{2}\right\} \leq c_{f}^{2} E\left\{\|\mathbf{V}\|_{\mathcal{V}}^{2}\right\}$. Using Eq. (118) yields $c_{K}^{2}\|\mathbf{V}\|_{\mathbb{V}}^{4} \leq c_{f}^{2}\|\mathbf{V}\|_{\vee}^{2}$ which can be rewritten as $\|\mathbf{V}\|_{\mathbb{V}} \leq c<+\infty$ with $c=c_{f} / c_{K}$. The proof of the uniqueness is straightforward because, if $\mathbf{V}$ and $\mathbf{V}^{\prime}$ are two solutions in $\mathbb{V}$, Eqs. (113) and (118) yield $\left\|\mathbf{V}-\mathbf{V}^{\prime}\right\|_{\mathbb{V}}^{2}=0$, i.e., $\mathbf{V}=\mathbf{V}^{\prime}$ in $\mathbb{V}$. Finally, since the bilinear form $(\mathbf{V}, \mathbf{W}) \mapsto \mathcal{K}(\mathbf{V}, \mathbf{W})$ is continous on $\mathbb{V} \times \mathbb{V}$ (see Section 5.5) there is a linear operator $\mathbb{K}$ which is continuous from $\mathbb{V}$ to $\mathbb{V}^{\prime}$ such that $\mathbb{K} \mathbf{V}=f$, in which $f$ is given in $\mathbb{V}^{\prime}$. Since the equation $\mathbb{K} \mathbf{V}=f$ has a unique solution in $\mathbb{V}$ (see the proof above), this means that $\mathbb{K}$ is invertible from $\mathbb{V}^{\prime}$ to $\mathbb{V}$ and consequently, is continuous from $\mathbb{V}^{\prime}$ to $\mathbb{V}$.

\section{Stochastic finite element}

\subsection{Finite element discretization}

The usual finite element method $[10,91]$ is utilized for discretizing the deterministic Eq. (106) and the random Eq. (113) which yields

$$
[\underline{K}] \underline{\mathbf{d}}=\mathbf{f} \quad, \quad[\mathbf{K}] \mathbf{D}=\mathbf{f} \quad,
$$

in which the deterministic stiffness matrix $[\underline{K}]$ and the random stiffness matrix $[\mathbf{K}]$ correspond to the finite element discretization of the bilinear forms $\underline{k}(\mathbf{v}, \mathbf{w})$ defined by Eq. (111) and $\mathbf{K}(\mathbf{v}, \mathbf{w})$ defined by Eq. (112). The deterministic vector $\mathbf{f}$ corresponds to the discretization of the linear form defined by Eq. (107). The deterministic vector $\underline{\mathbf{d}}$ and the random vector $\mathbf{D}$ are the vectors of the degrees of freedom (values of the deterministic displacement field $\mathbf{x} \mapsto \underline{\mathbf{v}}(\mathbf{x})$ of the mean model and 
values of the random displacement field $\mathbf{x} \mapsto \mathbf{V}(\mathbf{x})$ of the stochastic model, at the free nodes of the finite element mesh).

For a given finite element mesh of domain $\Omega$, let $\mathbf{x}^{1}, \ldots, \mathbf{x}^{N}$ be the set of all the integrating points of the finite elements of the model. The random field $\left\{\left[\mathbf{A}_{n}(\mathbf{x})\right], \mathbf{x} \in \Omega\right\}$ is then discretized in the integrating points of each finite element of the model. Consequently, the random stiffness matrix $[\mathbf{K}]$ can be rewritten as

$$
[\mathbf{K}]=\left[K\left(\left[\mathbf{A}_{n}\left(\mathbf{x}^{1}\right)\right], \ldots,\left[\mathbf{A}_{n}\left(\mathbf{x}^{N}\right)\right]\right)\right]
$$

in which $\left(\left[a_{1}\right], \ldots,\left[a_{N}\right]\right) \mapsto\left[K\left(\left[a_{1}\right], \ldots,\left[a_{N}\right]\right)\right]$ is a deterministic function which is completely defined.

\subsection{Constructing and solving the random matrix equation}

The random matrix equation $[\mathbf{K}] \mathbf{D}=\mathbf{f}$ (see Eq. (123)) can be solved by several methods, in particular by using the Monte Carlo numerical method [61].

For each realization $\theta \in \Theta$, we have the following steps.

(i) The realizations $\left\{\left[\mathbf{A}_{n}\left(\mathbf{x}^{1}, \theta\right)\right], \ldots,\left[\mathbf{A}_{n}\left(\mathbf{x}^{N}, \theta\right)\right]\right\}$ of random matrices $\left\{\left[\mathbf{A}_{n}\left(\mathbf{x}^{1}\right)\right], \ldots,\left[\mathbf{A}_{n}\left(\mathbf{x}^{N}\right)\right]\right\}$ are constructed by using Section 4.2(vii).

(ii) The realization of each finite element stiffness matrix is constructed and the assemblage of all these finite element matrices yields the realization $[\mathbf{K}(\theta)]$ of the random matrix $[\mathbf{K}]$ such that $[\mathbf{K}(\theta)]=\left[K\left(\left[\mathbf{A}_{n}\left(\mathbf{x}^{1}, \theta\right)\right], \ldots,\left[\mathbf{A}_{n}\left(\mathbf{x}^{N}, \theta\right)\right]\right)\right]$.

(iii) The linear matrix equation $[\mathbf{K}(\theta)] \mathbf{D}(\theta)=\mathbf{f}$ is solved to obtain $\mathbf{D}(\theta)$ and the realization $\mathbf{O}(\theta)$ of any observation $\mathbf{O}$ can be deduced.

Finally, the mathematical statistics are used for estimating the probability quantities related to random vectors $\mathbf{D}$ and $\mathbf{O}$. Convergence of the estimators with respect to the number of realizations has to be controlled.

\section{Class of random fields which can be approximated with the ensemble $\mathrm{SFE}^{+}$and their experimental identification}

The objective of this section is to introduce some remarks concerning (1) the class of random fields which can be approximated by a random field belonging to the ensemble $\mathrm{SFE}^{+}$and (2) some

C. Soize - CMAME - revised version December 2004 
remarks concerning the experimental identification of such a random field in solving a stochastic inverse problem.

Let $\mathbf{x} \mapsto\left[\mathbf{A}_{n}(\mathbf{x})\right]$ be a random field in $\mathrm{SFE}^{+}$, defined on probability space $(\Theta, \mathcal{T}, P)$, indexed by $\Omega$, with values in $M_{n}^{+}(\mathbb{R})$. In this section ( Section 7), we limit the presentation to the homogeneous case presented in Section 4.3. Consequently, in this section, $\mathbf{x} \mapsto\left[\mathbf{A}_{n}(\mathbf{x})\right]$ is viewed as the restriction to $\Omega$ of a homogeneous random field indexed by $\mathbb{R}^{d}$.

7.1. Summary of the parameters allowing a homogeneous random field in $\mathrm{SFE}^{+}$to be completely defined

From Sections 3 and 4, it can be deduced that the parameters allowing a homogeneous random field $\mathbf{x} \mapsto\left[\mathbf{A}_{n}(\mathbf{x})\right]$ indexed by $\mathbb{R}^{d}$ with values in $\mathrm{M}_{n}^{+}(\mathbb{R})$ and belonging to $\mathrm{SFE}^{+}$, to be completely defined are the following.

(i) The autocorrelation function $\boldsymbol{\eta} \mapsto R_{U}(\boldsymbol{\eta})$ of the random germ $U$ in $\mathcal{E}_{U}$ has to be such that $R_{U}(0)=1, R_{U}$ is a continuous function from $\mathbb{R}^{d}$ into $\mathbb{R}$, Eq. (6) holds and the power spectral density function $\mathbf{k} \mapsto S_{U}(\mathbf{k})$ has a compact support $\left[-K_{1}, K_{1}\right] \times \ldots \times\left[-K_{d}, K_{d}\right]$. It should be noted that the $d$ positive real numbers $K_{1}, \ldots, K_{d}$ can be defined by the $d$ spatial correlation lengths $L_{1}^{U}, \ldots, L_{d}^{U}$ given by Eq. (9).

(ii) The mean value $\left[\underline{a}_{n}\right]=E\left\{\left[\mathbf{A}_{n}(\mathbf{x})\right]\right\} \in \mathbb{M}_{n}^{+}(\mathbb{R})$ is independent of $\mathbf{x}$.

(iii) The parameter $\delta$ is defined by Eq. (37) and verifies Eq. (28), allowing the dispersion level to be controlled.

\subsection{Some properties of a random field in $\mathrm{SFE}^{+}$}

In this section, we summarize the second-order parameters and the fundamendal property of a homogeneous random field $\mathbf{x} \mapsto\left[\mathbf{A}_{n}(\mathbf{x})\right]$ indexed by $\mathbb{R}^{d}$ with values in $\mathbb{M}_{n}^{+}(\mathbb{R})$ and belonging to $\mathrm{SFE}^{+}$. The second-order properties and the fundamental property are the following.

(i) The mean value $\left[\underline{a}_{n}\right]=E\left\{\left[\mathbf{A}_{n}(\mathbf{x})\right]\right\} \in \mathbb{M}_{n}^{+}(\mathbb{R})$ is independent of $\mathbf{x}$.

(ii) The dispersion parameter $\delta_{A_{n}}$ defined by Eq. (87) and given by Eq. (93) is a function of $\delta$ and $\left[\underline{a}_{n}\right]$.

(iii) The fourth-order tensor-valued covariance function $\boldsymbol{\eta} \mapsto C^{A_{n}}(\mathbf{x}+\boldsymbol{\eta}, \mathbf{x})$ on $\mathbb{R}^{d}$ is defined by Eq. (82).

C. Soize - CMAME - revised version December 2004 
(iv) The $d$ spatial correlation lengths $L_{1}^{A_{n}}, \ldots, L_{d}^{A_{n}}$ are defined by Eq. (97).

(v) For a random field in $\mathrm{SFE}^{+}$, the ellipticity property is defined by Eq. (118) in which the random bilinear form $\mathbf{K}(\mathbf{v}, \mathbf{w})$ is defined by Eq. (112). As explained in Section 5.8, this fundamental property allows the stochastic boundary value problem related to the stochastic partial differential operator defined by Eq. (4) to have a solution which is a second-order random field.

\subsection{First class of approximation}

(i) Setting the problem. The problematic is related to the information which is available for identifying such a random field $\mathbf{x} \mapsto\left[\mathbf{A}_{n}(\mathbf{x})\right]$ with experimental data. As we have explained in Section 1, such a random field is constituted of 21 mutually dependent random fields. The available information is constituted of the parameters which can be experimentally identified by using mathematical statistics. For instance and in general, the system of the marginal probability distributions for the 21 mutually dependent random fields do not constitute an available information because the experimental database which is required for such an estimation is not available. This is the reason why the class $\mathrm{SFE}^{+}$of random fields having a small number of parameters has been constructed in Sections 3 and 4. The first class of approximation presented in this section corresponds to the larger class, that is to say, corresponds to the smallest number of parameters which can experimentally be identified.

(ii) Available information. Let $\mathbf{x} \mapsto\left[\mathbf{A}_{n}^{\exp }(\mathbf{x})\right]$ be the random field indexed by $\Omega \subset \mathbb{R}^{d}$ with values in $\mathbb{M}_{n}^{+}(\mathbb{R})$ which has to be approximated and for which the available information is the following (see Section 7.3.v-3 explaining why the available information has to be limited to the quantities defined in (a) to (d) below).

(a) The mean value $\left[\underline{a}_{n}^{\exp }\right]=E\left\{\left[\mathbf{A}_{n}^{\exp }(\mathbf{x})\right]\right\} \in \mathbb{M}_{n}^{+}(\mathbb{R})$ is independent of $\mathbf{x}$.

(b) The dispersion parameter $\delta_{A_{n}^{\exp }}$ is defined by Eq. (87).

(c) The $d$ spatial correlation lengths $L_{1}^{A_{n}^{\exp }}, \ldots, L_{d}^{A_{n}^{\exp }}$ are defined by Eq. (97) in which $r^{A_{n}^{\exp }}(\boldsymbol{\eta})$ is defined by Eq. (94).

(d) The random field $\mathbf{x} \mapsto\left[\mathbf{A}_{n}^{\exp }(\mathbf{x})\right]$ verifies an ellipticity condition in order that the stochastic boundary value problem has a second-order stochastic solution.

(iii) Approximation with a random field belonging to the ensemble $\mathrm{SFE}^{+}$. A random field $\mathbf{x} \mapsto$ $\left[\mathbf{A}_{n}^{\exp }(\mathbf{x})\right]$ whose available information is defined in Section 7.3.ii can be approximated by the restriction to the domain $\Omega$ of a homogeneous random field $\mathbf{x} \mapsto\left[\mathbf{A}_{n}(\mathbf{x})\right]$ indexed by $\mathbb{R}^{d}$ with values 
in $\mathbb{M}_{n}^{+}(\mathbb{R})$ and belonging to the ensemble $\mathrm{SFE}^{+}$, for which the random field germ $U$ is any random field belonging to $\mathcal{E}_{U}$ (for instance, the random field $U$ defined in Section 3.2.2).

(iv) Calculation of the parameters of the approximated random field. Taking into account Section 7.1, the random field $\mathbf{x} \mapsto\left[\mathbf{A}_{n}(\mathbf{x})\right]$ which approximates the random field $\mathbf{x} \mapsto\left[\mathbf{A}_{n}^{\exp }(\mathbf{x})\right]$ is then completely defined and its parameters can be calculated as follows.

(a) The mean value is such that $\left[\underline{a}_{n}\right]=\left[\underline{a}_{n}^{\exp }\right] \in \mathbb{M}_{n}^{+}(\mathbb{R})$.

(b) The dispersion parameter $\delta$ defined by Eq. (37) is calculated by using Eq. (88),

$$
\delta_{A_{n}^{\exp }}=\frac{\delta}{\sqrt{n+1}}\left\{1+\frac{\left(\operatorname{tr}\left[\underline{a}_{n}^{\exp }\right]\right)^{2}}{\operatorname{tr}\left\{\left[\underline{a}_{n}^{\exp }\right]^{2}\right\}}\right\}^{1 / 2} .
$$

(c) The spatial correlation lengths $L_{1}^{U}, \ldots, L_{d}^{U}$, defined by Eq. (9), are calculated by solving the following system of nonlinear algebraic equations

$$
\begin{aligned}
L_{1}^{A_{n}}\left(L_{1}^{U}, \ldots, L_{d}^{U}\right) & =L_{1}^{A_{n}^{\exp }} \\
\ldots & =\ldots \\
L_{d}^{A_{n}}\left(L_{1}^{U}, \ldots, L_{d}^{U}\right) & =L_{d}^{A_{n}^{\exp }},
\end{aligned}
$$

in which, for all $j$ in $\{1, \ldots, d\}$, the mapping $\left(L_{1}^{U}, \ldots, L_{d}^{U}\right) \mapsto L_{j}^{A_{n}}\left(L_{1}^{U}, \ldots, L_{d}^{U}\right)$ is defined by Eq. (97) with the model of the random field $\mathbf{x} \mapsto\left[\mathbf{A}_{n}(\mathbf{x})\right]$ defined in Sections 3 and 4 . In pratice, it should be noted that, for all $j$ in $\{1, \ldots, d\}, L_{j}^{U}$ is closed to $L_{j}^{A_{n}}$ (see for instance Section 8). This means that the system of nonlinear algebraic equations defined by Eq. (126) allowing $L_{1}^{U}, \ldots, L_{d}^{U}$ to be calculated can be solved by iteration, the mapping $\left(L_{1}^{U}, \ldots, L_{d}^{U}\right) \mapsto\left(L_{1}^{A_{n}}, \ldots, L_{d}^{A_{n}}\right)$ being easily calculated by using the construction defined in Sections 3 and 4 with a given random field germ $U$ in $\mathcal{E}_{U}$ (for instance with the random field defined in Section 3.2.2).

(v) Statistical estimation of the available information. In this subsection, we show how the available information defined in Section 7.3.ii can be estimated by using mathematical statistics. Let $\mathbf{x}^{1}, \ldots, \mathbf{x}^{N}$ be $N$ points in $\Omega \subset \mathbb{R}^{d}$. It is assumed that $\nu>1$ independent realizations $\left[\mathbf{A}_{n}^{\exp }\left(\mathbf{x}^{k}, \theta_{\ell}\right)\right]$ for $k=1, \ldots, N$ and $\theta_{\ell} \in \Theta$ with $\ell=1, \ldots, \nu$ are known from experiments.

(v-1) Estimations. In this subsection, in order to limit the developments, we directly give an estimation of each quantity to be estimated without defining the sequence of estimators and without studying the properties of such a sequence of estimators (see $[35,38,55,64,72])$. Nevertheless, the 
convergence of each estimator is fundamental to construct a good estimation. Some important comments concerning this aspect are given in the following subsection 7.3.v-2.

An estimation $\left[\widehat{a}_{\nu, N}^{\exp }\right]$ of the mean value $\left[\underline{a}_{n}^{\exp }\right]=E\left\{\left[\mathbf{A}_{n}^{\exp }(\mathbf{x})\right]\right\}$ of the random field $\mathbf{x} \mapsto\left[\mathbf{A}_{n}^{\exp }(\mathbf{x})\right]$ can be written as

$$
\left[\widehat{a}_{\nu, N}^{\exp }\right]=\frac{1}{N \nu} \sum_{k=1}^{N} \sum_{\ell=1}^{\nu}\left[\mathbf{A}_{n}^{\exp }\left(\mathbf{x}^{k}, \theta_{\ell}\right)\right]
$$

An estimation $\boldsymbol{\eta} \mapsto \widehat{C}_{\nu, N}^{\text {exp }}(\boldsymbol{\eta})$ of the fourth-order tensor-valued covariance function $\boldsymbol{\eta} \mapsto C^{\mathbf{A}_{n}^{\exp }}(\boldsymbol{\eta})=$ $E\left\{\left(\left[\mathbf{A}_{n}^{\exp }(\mathbf{x}+\boldsymbol{\eta})\right]-\left[\underline{a}_{n}^{\exp }\right]\right) \otimes\left(\left[\mathbf{A}_{n}^{\exp }(\mathbf{x})\right]-\left[\underline{a}_{n}^{\exp }\right]\right)\right\}$ can be written as

$$
\widehat{C}_{\nu, N}^{\exp }\left(\boldsymbol{\eta}^{k k^{\prime}}\right)=\frac{1}{\nu} \sum_{\ell=1}^{\nu}\left(\left[\mathbf{A}_{n}^{\exp }\left(\mathbf{x}^{k}, \theta_{\ell}\right)\right]-\left[\widehat{a}_{\nu, N}^{\exp }\right]\right) \otimes\left(\left[\mathbf{A}_{n}^{\exp }\left(\mathbf{x}^{k^{\prime}}, \theta_{\ell}\right)\right]-\left[\widehat{a}_{\nu, N}^{\exp }\right]\right),
$$

in which

$$
\boldsymbol{\eta}^{k k^{\prime}}=\mathbf{x}^{k}-\mathbf{x}^{k^{\prime}} \quad, \quad\left(k, k^{\prime}\right) \in\{1, \ldots, N\} \times\{1, \ldots, N\}
$$

An estimation $\boldsymbol{\eta} \mapsto \widehat{r}_{\nu, N}^{\exp }(\boldsymbol{\eta})$ of the function $\boldsymbol{\eta} \mapsto r^{\mathbf{A}_{n}^{\exp }}(\boldsymbol{\eta})$ from $\mathbb{R}^{d}$ into $\mathbb{R}$ and defined by Eq. (94) can be written as

$$
\widehat{r}_{\nu, N}^{\exp }\left(\boldsymbol{\eta}^{k k^{\prime}}\right)=\frac{1}{\nu \widehat{C}_{\nu, N}^{\exp }(0)} \sum_{\ell=1}^{\nu} \operatorname{tr}\left\{\left(\left[\mathbf{A}_{n}^{\exp }\left(\mathbf{x}^{k}, \theta_{\ell}\right)\right]-\left[\widehat{a}_{\nu, N}^{\exp }\right]\right)\left(\left[\mathbf{A}_{n}^{\exp }\left(\mathbf{x}^{k^{\prime}}, \theta_{\ell}\right)\right]-\left[\widehat{a}_{\nu, N}^{\exp }\right]\right)\right\}
$$

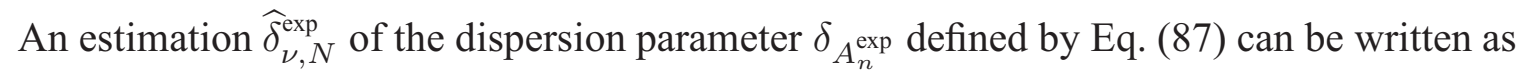

$$
\widehat{\delta}_{\nu, N}^{\exp }=\frac{1}{\left\|\left[\widehat{a}_{\nu, N}^{\exp }\right]\right\|_{F}}\left\{\frac{1}{N \nu} \sum_{k=1}^{N} \sum_{\ell=1}^{\nu}\left\|\left[\mathbf{A}_{n}^{\exp }\left(\mathbf{x}^{k}, \theta_{\ell}\right)\right]-\left[\widehat{a}_{\nu, N}^{\exp }\right]\right\|_{F}^{2}\right\}^{1 / 2}
$$

Since $\Omega \subset \mathbb{R}^{d}$ is a bounded domain and since $\mathbf{x}^{1}, \ldots, \mathbf{x}^{N}$ are any points in $\Omega$, a direct construction of an estimation $\widehat{L}_{1, \nu, N}^{\exp }, \ldots, \widehat{L}_{d, \nu, N}^{\exp }$ of the spatial correlation lengths $L_{1}^{A_{n}^{\exp }}, \ldots, L_{d}^{A_{n}^{\exp }}$ cannot be deduced from Eq. (97). For all $j$ fixed in $\{1, \ldots, d\}$, an estimation $\widehat{L}_{j, \nu, N}^{\exp }$ of $L_{j}^{A_{n}^{\exp }}$ can be constructed as the solution in $L_{j}$ of the following optimization problem

$$
\min _{L_{j}}\left\{\sum_{k=1}^{N} \sum_{k^{\prime}=1}^{N}|| \widehat{r}_{\nu, N}^{\exp }\left(0, \ldots, 0, x_{j}^{k}-x_{j}^{k^{\prime}}, 0, \ldots, 0\right)\left|-e^{-\left(x_{j}^{k}-x_{j}^{k^{\prime}}\right) / L_{j}}\right|\right\}
$$

in which the double summation over $k$ and $k^{\prime}$ is limited to the points such that $x_{j}^{k}-x_{j}^{k^{\prime}} \geq 0$.

(v-2) Convergence of the estimations. In the experimental context, the value of $\nu$ is generally small (limitation of the number of specimens which can experimentally be tested). Nevertheless, it is 
assumed that this small value of $\nu$ and the value of $N$ are such that the estimation $\left[\widehat{a}_{\nu, N}^{\exp }\right]$ defined by Eq. (127) is sufficiently converged (if not, then the estimation problem is an ill-posed problem). However, if the values of $\nu$ and $N$ allow the mean value $\left[\underline{a}_{n}^{\text {exp }}\right]$ to be reasonably estimated, these values do not generally allow the ellipticity property defined by Eq. (117) to be really tested or the fourthorder tensor-valued covariance function $\boldsymbol{\eta} \mapsto C^{A_{n}^{\exp }}(\boldsymbol{\eta})$ to be estimated by Eq. (128) with a good rate of convergence. Similarly, the function $\boldsymbol{\eta} \mapsto r^{A_{n}^{\exp }}(\boldsymbol{\eta})$ defined by Eq. (94) cannot be estimated by Eq. (130) with a good rate of convergence. However, if the estimation of $\boldsymbol{\eta} \mapsto r_{n}^{A_{n}^{\exp }}(\boldsymbol{\eta})$ is generally not converged, the estimation $\widehat{\delta}_{\nu, N}^{\exp }$ defined by Eq. (131) and the estimations $\widehat{L}_{1, \nu, N}^{\exp }, \ldots, \widehat{L}_{d, \nu, N}^{\exp }$ defined by Eq. (132) can be assumed to be converged.

(v-3) Limitation of the available information induced by the experimental identification context. In Section 7.3.ii, we have introduced the mean value, the dispersion parameter, the spatial correlation lengths and the ellipticity property as the available information. For applications and in the experimental identification context, this limitation of the available information is due to the lack of convergence of the estimators introduced in Section 7.3.v-1 because the number $\nu$ of independent realizations corresponds to the number of specimens which can experimentally be tested and which is then generally small.

\subsection{Second class of approximation}

(i) Setting the problem. The second class of approximation presented in this section corresponds to the class for which the parameters related to all the second-order quantities can experimentally be identified.

(ii) Available information. Let $\mathbf{x} \mapsto\left[\mathbf{A}_{n}^{\exp }(\mathbf{x})\right]$ be the random field indexed by $\Omega \subset \mathbb{R}^{d}$ with values in $M_{n}^{+}(\mathbb{R})$ which has to be approximated and for which the available information is the following.

(a)The mean value $\left[\underline{a}_{n}^{\exp }\right]=E\left\{\left[\mathbf{A}_{n}^{\exp }(\mathbf{x})\right]\right\} \in \mathbb{M}_{n}^{+}(\mathbb{R})$ is independent of $\mathbf{x}$.

(b) The fourth-order tensor-valued covariance function is given and is written as

$$
\boldsymbol{\eta} \mapsto C^{\mathbf{A}_{n}^{\exp }}(\boldsymbol{\eta})=E\left\{\left(\left[\mathbf{A}_{n}^{\exp }(\mathbf{x}+\boldsymbol{\eta})\right]-\left[\underline{a}_{n}^{\exp }\right]\right) \otimes\left(\left[\mathbf{A}_{n}^{\exp }(\mathbf{x})\right]-\left[\underline{a}_{n}^{\exp }\right]\right)\right\}
$$

(c) The random field $\mathbf{x} \mapsto\left[\mathbf{A}_{n}^{\exp }(\mathbf{x})\right]$ verifies an ellipticity condition in order that the stochastic boundary value problem has a second-order stochastic solution.

C. Soize - CMAME - revised version December 2004 
(d) It should be noted that, as soon as the function $\boldsymbol{\eta} \mapsto C^{\mathbf{A}_{n}^{\text {exp }}}(\boldsymbol{\eta})$ is known, then the dispersion parameter $\delta_{A_{n}^{\text {exp }}}$ defined by Eq. (87) and the function $\boldsymbol{\eta} \mapsto r^{A_{n}^{\text {exp }}}(\boldsymbol{\eta})$ defined by Eq. (94) can be calculated.

(iii) Approximation with a random field belonging to the ensemble $\mathrm{SFE}^{+}$. A random field $\mathbf{x} \mapsto$ $\left[\mathbf{A}_{n}^{\exp }(\mathbf{x})\right]$ whose available information is defined in Section 7.4.ii can be approximated by the restriction to the domain $\Omega$ of a homogeneous random field $\mathbf{x} \mapsto\left[\mathbf{A}_{n}(\mathbf{x})\right]$ indexed by $\mathbb{R}^{d}$, with values in $\mathrm{M}_{n}^{+}(\mathbb{R})$ and belonging to the ensemble $\mathrm{SFE}^{+}$, for which the random field germ $U$ is a random field in $\mathcal{E}_{U}$ and for which the autocorrelation function $\boldsymbol{\eta} \mapsto R_{U}(\boldsymbol{\eta})$ has to be constructed (see Section 7.4.iv.

(iv) Calculation of the parameters of the approximated random field. Taking into account Section 7.1, the random field $\mathbf{x} \mapsto\left[\mathbf{A}_{n}(\mathbf{x})\right]$ which approximates the random field $\mathbf{x} \mapsto\left[\mathbf{A}_{n}^{\exp }(\mathbf{x})\right]$ is then completely defined and its parameters can be calculated as follows.

(a) The mean value is such that $\left[\underline{a}_{n}\right]=\left[\underline{a}_{n}^{\exp }\right] \in M_{n}^{+}(\mathbb{R})$.

(b) The dispersion parameter $\delta$ defined by Eq. (37) is calculated by using Eq. (125).

(c) For a given function $\boldsymbol{\eta} \mapsto R_{U}(\boldsymbol{\eta})$ from $\mathbb{R}^{d}$ into $\mathbb{R}$ such as the random field germ $U$ belongs to $\mathcal{E}_{U}$ (see Section 3.2.1), the function $\boldsymbol{\eta} \mapsto r^{\mathbf{A}_{n}}\left(\boldsymbol{\eta} ; R_{U}\right)$ from $\mathbb{R}^{d}$ into $\mathbb{R}$ defined by Eq. (94) can be calculated by using the model of the random field $\mathbf{x} \mapsto\left[\mathbf{A}_{n}(\mathbf{x})\right]$ defined in Sections 3 and 4. Consequently, $R_{U}$ can be constructed as the solution of the following optimization problem,

$$
\min _{R_{U}} \int_{\mathbb{R}^{d}}\left|r^{\mathbf{A}_{n}^{\text {exp }}}(\boldsymbol{\eta})-r^{\mathbf{A}_{n}}\left(\boldsymbol{\eta} ; R_{U}\right)\right|^{2} d \boldsymbol{\eta}
$$

in which $R_{U}$ has to verify the properties defined in Section 3.2.1 in order that $U$ belongs to $\mathcal{E}_{U}$.

(v) Statistical estimation of the available information. In this subsection, it is shown how the available information defined in Section 7.4.ii can be estimated by using mathematical statistics. Let $\mathbf{x}^{1}, \ldots, \mathbf{x}^{N}$ be $N$ points in $\Omega \subset \mathbb{R}^{d}$. For $k=1, \ldots, N$, it is assumed that $\nu$ independent realizations $\left\{\left[\mathbf{A}_{n}^{\exp }\left(\mathbf{x}^{k}, \theta_{\ell}\right)\right], \theta_{\ell} \in \Theta, \ell=1, \ldots, \nu\right\}$ are known from experiments.

(v-1) Estimations. An estimation $\left[\widehat{a}_{\nu, N}^{\exp }\right]$ of the mean value $\left[\underline{a}_{n}^{\exp }\right]=E\left\{\left[\mathbf{A}_{n}^{\exp }(\mathbf{x})\right]\right\}$ of the random field $\mathbf{x} \mapsto\left[\mathbf{A}_{n}^{\exp }(\mathbf{x})\right]$ is given by Eq. (127). An estimation $\boldsymbol{\eta} \mapsto \widehat{C}_{\nu, N}^{\exp }(\boldsymbol{\eta})$ of the fourth-order tensor-valued covariance function $\boldsymbol{\eta} \mapsto C_{n}^{\mathbf{A}_{n}^{\exp }}(\boldsymbol{\eta})$ defined by Eq. (133) is given by Eqs. (128) and (129). An estimation $\boldsymbol{\eta} \mapsto \widehat{r}_{\nu, N}^{\exp }(\boldsymbol{\eta})$ of $\boldsymbol{\eta} \mapsto r^{\mathbf{A}_{n}^{\exp }}(\boldsymbol{\eta})$ is given by Eqs. (130) and (129). An estimation $\widehat{\delta}_{\nu, N}^{\exp }$ 
of $\delta_{A_{n}^{\exp }}$ defined by Eq. (87) is given by Eq. (131). Since $\Omega \subset \mathbb{R}^{d}$ is a bounded domain and since $\mathbf{x}^{1}, \ldots, \mathbf{x}^{N}$ are any $N$ points in $\Omega$, the following approximation of Eq. (134) is introduced

$$
\min _{R_{U}} \sum_{k=1}^{N} \sum_{k^{\prime}=1}^{N}\left|r^{\mathbf{A}_{n}^{\exp }}\left(\mathbf{x}^{k}-\mathbf{x}^{k^{\prime}}\right)-r^{\mathbf{A}_{n}}\left(\mathbf{x}^{k}-\mathbf{x}^{k^{\prime}} ; R_{U}\right)\right|^{2}
$$

in which $R_{U}$ have to verify the properties defined in Section 3.2.1 in order that $U$ belongs to $\mathcal{E}_{U}$.

(v-2) Convergence of the estimations. For this second class of approximation, it is assumed that $\nu$ is sufficiently large for that the convergence of the estimation defined by Eqs. (128)-(129) is reached.

\section{(v-3) Limitation of the second class of approximation with respect to the experimental identification} context. In Section 7.4.ii, we have introduced the available information constituted of the mean value, the fourth-order tensor-valued covariance function, and the ellipticity property. In the experimental identification context, the convergence of the estimation of the fourth-order tensor-valued covariance function has to be reached which means that the number of specimens which can experimentally be tested has to be sufficiently large.

\subsection{Difficulties related to the construction of the realizations of the random field to be identified}

In Sections 7.3.v and 7.4.v, for $k=1, \ldots, N$, it is assumed that $\nu$ independent realizations $\left\{\left[\mathbf{A}_{n}^{\exp }\left(\mathbf{x}^{k}, \theta_{\ell}\right)\right], \theta_{\ell} \in \Theta, \ell=1, \ldots, \nu\right\}$ are known (from experiments) in which $\mathbf{x}^{1}, \ldots, \mathbf{x}^{N}$ are $N$ points in $\Omega \subset \mathbb{R}^{d}$. Each realization $\left\{\left[\mathbf{A}_{n}^{\exp }\left(\mathbf{x}^{k}, \theta_{\ell}\right)\right], \theta_{\ell} \in \Theta\right\}$ is associated with a given specimen $\ell$ which is experimentally tested. In practice, such a realization is not directly obtained from the measurements but is indirectly constructed by using a model as follows. Let us assume that the random field $\mathbf{x} \mapsto\left[\mathbf{A}_{n}(\mathbf{x})\right]$ (with $n=6$ ) models the fourth-order elasticity tensor of a non homogeneous anisotropic linear elastic random material. A set of $\nu$ "identical" specimens are manufactured with this material using the same process. The mechanical-mathematical model of these specimens is the boundary value problem defined in Section 5. Each specimen occupies the same bounded domain $\Omega$ with the same boundary conditions. For each specimen, the experimental test consists in measuring the displacement field on a part $\Gamma_{b}$ of the boundary $\Gamma$ due to the same set of $Q$ external loads applied to the other part $\Gamma_{g}$ of the boundary $\Gamma$. The finite element model defined by Eq. (123) is used. The random vector $\mathbf{D}$ of the degrees of freedom (DOF) is then written as $\mathbf{D}=\left(\mathbf{D}_{b}, \mathbf{D}_{i}\right)$ in which $\mathbf{D}_{b}$ is the vector of the $m_{b}$ measured DOF in $\Gamma_{b}$ and where $\mathbf{D}_{i}$ is the vector of the other DOF. From Eqs. (123) and (124), it can be deduced that, for each applied external load 
$q$, the displacement of the degrees of freedom in $\Gamma_{b}$ is the random vector denoted as $\mathbf{D}_{b, q}$ and which can be written as

$$
\mathbf{D}_{b, q}=\mathbf{b}\left(\left[\mathbf{A}_{n}\left(\mathbf{x}^{1}\right)\right], \ldots,\left[\mathbf{A}_{n}\left(\mathbf{x}^{N}\right)\right] ; q\right)
$$

where $\left.\left(\left[a_{1}\right], \ldots,\left[a_{N}\right]\right) \mapsto \mathbf{b}\left(\left[a_{1}\right], \ldots,\left[a_{N}\right]\right) ; q\right)$ is a deterministic function which can easily be constructed and which depends on the applied external load $q$. The measurements are then constituted of the familly of vectors $\mathbf{D}_{b, q}^{\exp }\left(\theta_{\ell}\right)$ for $\ell=1, \ldots, \nu$ (the $\nu$ specimens) and for $q=$ $1, \ldots, Q$ (the $Q$ applied external loads). In general, $\mathbf{D}_{b, 1}^{\exp }, \ldots, \mathbf{D}_{b, Q}^{\exp }$ are mutually dependent random vectors but $\mathbf{D}_{b, 1}^{\exp }\left(\theta_{\ell}\right), \ldots, \mathbf{D}_{b, Q}^{\exp }\left(\theta_{\ell}\right)$ are assumed to be algebraically independent. For $\ell$ fixed, $\left[\mathbf{A}_{n}^{\exp }\left(\mathbf{x}^{1}, \theta_{\ell}\right)\right], \ldots,\left[\mathbf{A}_{n}^{\exp }\left(\mathbf{x}^{N}, \theta_{\ell}\right)\right]$ can be constructed as the solution in $\left[a_{1}\right], \ldots,\left[a_{N}\right]$ of the following optimization problem,

$$
\min _{\left[a_{1}\right] \in \mathbb{M}_{n}^{+}(\mathbb{R}), \ldots,\left[a_{N}\right] \in \mathbb{M}_{n}^{+}(\mathbb{R})} \sum_{q=1}^{Q}\left\|\mathbf{D}_{b, q}^{\exp }\left(\theta_{\ell}\right)-\mathbf{b}\left(\left[a_{1}\right], \ldots,\left[a_{N}\right] ; q\right)\right\|^{2} .
$$

Such a method can be used if the optimization problem defined by Eq. (137) has a unique solution. Clearly, a necessary, but not sufficient condition, is

$$
Q \times m_{b} \gg 21 \times N
$$

in which the factor 21 corresponds to the independent entries of each symmetric $(6 \times 6)$ real matrix $\left[a_{k}\right]$. In general, $Q$ is small (for instance 2, 3 or 4 ) and $N$ is large (for instance 1000,10000 or 100000 ). Let us assume that $\Omega$ is a box for which the finite element model is a regular mesh of $(n+1) \times(m+1) \times(p+1)$ nodes and $n \times m \times p$ finite elements which are 8-nodes solid elements with $2 \times 2 \times 2$ integrating points. Therefore, we have $N=8 \times n \times m \times p$. Assuming that all the DOF of the boundary are measured, we then have $m_{b}=6 \times(n+m) \times(p+1)$. The criterion defined by Eq. (138) can then be rewritten as

$$
\frac{Q}{28} \frac{(p+1)}{p} \gg \frac{n \times m}{n+m}
$$

For $Q=4$, Eq. (139) is never verified. More generally, for regular finite element mesh of the domain $\Omega$, the criterion defined by Eq. (138) is not verified. These potential difficulties lead us to introduce below another experimental identification of the random field $\mathbf{x} \mapsto\left[\mathbf{A}_{n}(\mathbf{x})\right]$ without using the realizations $\left[\mathbf{A}_{n}^{\exp }\left(\mathbf{x}^{1}, \theta_{\ell}\right)\right], \ldots,\left[\mathbf{A}_{n}^{\exp }\left(\mathbf{x}^{N}, \theta_{\ell}\right)\right]$.

7.6. Experimental identification with the first class of approximation without using the realizations of the random field which has to be identified 
(i) Simulating the random responses with the first class of approximation of the random field which has to be identified. The hypotheses introduced in Section 7.5 and the finite element model defined by Eq. (123) are used. The random vector $\mathbf{D}$ is always written as $\mathbf{D}=\left(\mathbf{D}_{b}, \mathbf{D}_{i}\right)$ and $\mathbf{D}_{b, q}$ is the random vector of the measured displacements induced by the applied external load $q$. For the first class of approximation, the random field $\mathbf{x} \mapsto\left[\mathbf{A}_{n}(\mathbf{x})\right]$ is completely defined by the parameters defined in Section 7.1 in which the random field germ $U$ is defined in Section 3.2.1. Consequently, the random field $\mathbf{x} \mapsto\left[\mathbf{A}_{n}(\mathbf{x})\right]$ is completely defined by the following parameters: the mean value $\left[\underline{a}_{n}\right]=E\left\{\left[\mathbf{A}_{n}(\mathbf{x})\right]\right\} \in \mathbb{M}_{n}^{+}(\mathbb{R})$ which is independent of $\mathbf{x}$, the parameter $\delta$ defined by Eq. (37) and verifying Eq. (28) and the $d$ spatial correlation lengths $L_{1}^{U}, \ldots, L_{d}^{U}$ defined by Eq. (9). Let $\boldsymbol{\lambda}$ representing the set of the parameters $\left[\underline{a}_{n}\right], \delta$ and $L_{1}^{U}, \ldots, L_{d}^{U}$,

$$
\boldsymbol{\lambda}=\left\{\left[\underline{a}_{n}\right], \delta, L_{1}^{U}, \ldots, L_{d}^{U}\right\} .
$$

The random vetor $\mathbf{D}_{b, q}$ can then be written as

$$
\mathbf{D}_{b, q}=\mathbf{B}(\boldsymbol{\lambda} ; q)
$$

in which $\boldsymbol{\lambda} \mapsto \mathbf{B}(\boldsymbol{\lambda} ; q)$ is a random function which can easily be constructed with Eq. (123) and with Sections 3 and 4 . For any $\theta$ in $\Theta$, the realization $\mathbf{D}_{b, q}(\theta)$ of random vector $\mathbf{D}_{b, q}$ is given by

$$
\begin{aligned}
\mathbf{D}_{b, q}(\theta) & =\mathbf{B}(\boldsymbol{\lambda} ; q ; \theta) \\
& =\mathbf{b}\left(\left[\mathbf{A}_{n}\left(\mathbf{x}^{1}, \theta\right)\right], \ldots,\left[\mathbf{A}_{n}\left(\mathbf{x}^{N} \theta\right)\right] ; q\right),
\end{aligned}
$$

in which $\left[\mathbf{A}_{n}\left(\mathbf{x}^{1}, \theta\right)\right], \ldots,\left[\mathbf{A}_{n}\left(\mathbf{x}^{N} \theta\right)\right]$ depend only on $\boldsymbol{\lambda}$. Equation (142) allows any probabilistic quantity related to the random vector $\mathbf{D}_{b, q}$ to be estimated by using the Monte Calo numerical simulation and the mathematical statistics. The mean vector and the correlation matrix are defined by

$$
\mathbf{m}_{\mathbf{D}_{b, q}}(\boldsymbol{\lambda} ; q)=E\left\{\mathbf{D}_{b, q}\right\} \quad, \quad\left[R_{\mathbf{D}_{b, q}}(\boldsymbol{\lambda} ; q)\right]=E\left\{\mathbf{D}_{b, q} \mathbf{D}_{b, q}^{T}\right\} \quad .
$$

The variation index is defined by

$$
\delta_{\mathbf{D}_{b, q}}(\boldsymbol{\lambda} ; q)=\frac{\left(E\left\{\left\|\mathbf{D}_{b, q}-\mathbf{m}_{\mathbf{D}_{b, q}}\right\|^{2}\right\}\right)^{1 / 2}}{\left\|\mathbf{m}_{\mathbf{D}_{b, q}}\right\|} .
$$

Let us introduce the random vector $\mathbb{D}=\left(\mathbf{D}_{b, 1}, \ldots, \mathbf{D}_{b, Q}\right)$ whose components $\mathbf{D}_{b, 1}, \ldots, \mathbf{D}_{b, Q}$ are mutually dependent random vectors. The probability distribution $P_{\mathbb{D}}$ of the random vector $\mathbb{D}$ is assumed to be defined by a probability density function with respect to the Lebesgue measure $d \boldsymbol{\mu}$ which is written as

$$
P_{\mathbb{D}}(d \boldsymbol{\mu} ; \boldsymbol{\lambda})=p_{\mathbb{D}}(\boldsymbol{\mu} ; \boldsymbol{\lambda}) d \boldsymbol{\mu} .
$$


(ii) Estimating the random responses with the measurements. The measurements are constituted of the familly of vectors $\mathbb{D}^{\exp }\left(\theta_{\ell}\right)=\left(\mathbf{D}_{b, 1}^{\exp }\left(\theta_{\ell}\right), \ldots, \mathbf{D}_{b, Q}^{\exp }\left(\theta_{\ell}\right)\right)$ for $\ell=1, \ldots, \nu$ (the $\nu$ specimens) and for $q=1, \ldots, Q$ (the $Q$ applied external loads). For a given applied external load $q$, an estimation $\widehat{\mathbf{m}}_{q, \nu}^{\exp }$ of the mean value $\mathbf{m}_{\mathbf{D}_{b, q}^{\exp }}$ of the random vector $\mathbf{D}_{b, q}^{\exp }$ can be written as

$$
\widehat{\mathbf{m}}_{q, \nu}^{\exp }=\frac{1}{\nu} \sum_{\ell=1}^{\nu} \mathbf{D}_{b, q}^{\exp }\left(\theta_{\ell}\right)
$$

For a given applied external load $q$, the estimations $\left[\widehat{R}_{q, \nu}^{\exp }\right]$ and $\widehat{\delta}_{q, \nu}^{\exp }$ of the correlation matrix $\left[R_{\mathbf{D}_{b, q}^{\exp }}\right]$ and the variation index $\delta_{\mathbf{D}_{b, q}^{\exp }}$ of the random vector $\mathbf{D}_{b, q}^{\exp }$ can be written as

$$
\begin{gathered}
{\left[\widehat{R}_{q, \nu}^{\exp }\right]=\frac{1}{\nu} \sum_{\ell=1}^{\nu} \mathbf{D}_{b, q}^{\exp }\left(\theta_{\ell}\right) \mathbf{D}_{b, q}^{\exp }\left(\theta_{\ell}\right)^{T},} \\
\delta_{\mathbf{D}_{b, q}^{\exp }}=\frac{1}{\left\|\widehat{\mathbf{m}}_{q, \nu}^{\exp }\right\|}\left(\frac{1}{\nu} \sum_{\ell=1}^{\nu}\left\{\left\|\mathbf{D}_{b, q}^{\exp }\left(\theta_{\ell}\right)-\widehat{\mathbf{m}}_{q, \nu}^{\exp }\right\|^{2}\right\}\right)^{1 / 2} .
\end{gathered}
$$

In the experimental identification context, there is generally a lack of convergence of the estimation defined by Eq. (147) due to a small value $\nu$ of specimens which can experimentally be tested. Nevertheless, it is assumed that this value of $\nu$ allows a convergence of the estimations defined by Eq. (146) and Eq. (148) to be reached.

(iii) Second-order moment method for estimating the parameters of the random field which has to be identified. The first method consists in performing the experimental identification of $\boldsymbol{\lambda}$ defined by Eq. (140) in solving the following optimization problem,

$$
\min _{\boldsymbol{\lambda} \in \mathcal{S}_{\boldsymbol{\lambda}}} \sum_{q=1}^{Q}\left(\delta_{\mathbf{D}_{b, q}^{\exp }}-\delta_{\mathbf{D}_{b, q}}(\boldsymbol{\lambda} ; q)\right)^{2}
$$

in which $\boldsymbol{\lambda}=\left\{\left[\underline{a}_{n}\right], \delta, L_{1}^{U}, \ldots, L_{d}^{U}\right\}$ runs though the set $\mathcal{S}_{\boldsymbol{\lambda}}$ defined by

$$
\mathcal{S}_{\lambda}=M_{n}^{+}(\mathbb{R}) \times\left[0, \delta_{n}\left[\times \mathbb{R}^{+} \times \ldots \times \mathbb{R}^{+}\right.\right.
$$

and where $\delta_{n}$ is defined by Eq. (28). 
(iv) Maximum likelihood method for estimating the parameters of the random field which has to be identified. We introduce the function $\boldsymbol{\lambda} \mapsto \mathcal{L}\left(\boldsymbol{\lambda} ; \mathbb{D}^{\exp }\left(\theta_{1}\right), \ldots, \mathbb{D}^{\exp }\left(\theta_{\nu}\right)\right)$ from $\mathcal{S}_{\boldsymbol{\lambda}}$ defined by Eq. (150) into $] 0,+\infty[$ such that

$$
\mathcal{L}\left(\boldsymbol{\lambda} ; \mathbb{D}^{\exp }\left(\theta_{1}\right), \ldots, \mathbb{D}^{\exp }\left(\theta_{\nu}\right)\right)=\Pi_{\ell=1}^{\nu} p_{\mathbb{D}}\left(\mathbb{D}^{\exp }\left(\theta_{\ell}\right) ; \boldsymbol{\lambda}\right)
$$

in which the probability density function $p_{\mathbb{D}}(\boldsymbol{\mu} ; \boldsymbol{\lambda})$ is defined by Eq. (145). We deduce that

$$
\log \left\{\mathcal{L}\left(\boldsymbol{\lambda} ; \mathbb{D}^{\exp }\left(\theta_{1}\right), \ldots, \mathbb{D}^{\exp }\left(\theta_{\nu}\right)\right)\right\}=\sum_{\ell=1}^{\nu} \log \left\{p_{\mathbb{D}}\left(\mathbb{D}^{\exp }\left(\theta_{\ell}\right) ; \boldsymbol{\lambda}\right)\right\}
$$

The maximum likelihood principle consists in estimating $\boldsymbol{\lambda}$ as the solution of the following optimization problem,

$$
\min _{\boldsymbol{\lambda} \in \mathcal{S}_{\boldsymbol{\lambda}}} \log \left\{\mathcal{L}\left(\boldsymbol{\lambda} ; \mathbb{D}^{\exp }\left(\theta_{1}\right), \ldots, \mathbb{D}^{\exp }\left(\theta_{\nu}\right)\right)\right\}
$$

in which $\mathcal{S}_{\lambda}$ is defined by Eq. (150).

(v) Experimental identification with the second class of approximation without using the realizations of the random field which has to be identified. The second-order moment method and the maximum likelihood method presented in Sections 7.6.iii and 7.6.iv for estimating the parameter $\left.\boldsymbol{\lambda}=\left\{\underline{a}_{n}\right], \delta, L_{1}^{U}, \ldots, L_{d}^{U}\right\}$ of the random field $\mathbf{x} \mapsto\left[\mathbf{A}_{n}(\mathbf{x})\right]$ with the first class of approximation can be used for estimating the parameter $\boldsymbol{\lambda}=\left\{\left[\underline{a}_{n}\right], \delta, R_{U}\right\}$ of the random field $\mathbf{x} \mapsto\left[\mathbf{A}_{n}(\mathbf{x})\right]$ with the second class of approximation, in substituting $\mathcal{S}_{\lambda}$ defined by Eq. (150) by

$$
\mathcal{S}_{\lambda}=\mathbb{M}_{n}^{+}(\mathbb{R}) \times\left[0, \delta_{n}\left[\times \mathcal{R}_{U}\right.\right.
$$

where $\delta_{n}$ is defined by Eq. (28) and where $\mathcal{R}_{U}$ is the set of all the functions $\boldsymbol{\eta} \mapsto R_{U}(\boldsymbol{\eta})$ verifying the properties defined in Section 3.2.1.

\section{Numerical example}

In this section, a numerical example is presented. It consists of a three-dimensional linear elastostatic problem. The mean model of the material is homogeneous and isotropic. The stochastic model is a random homogeneous and anisotropic linear elastic material whose elasticity tensor is a fourth-order tensor-valued random field depending on the three coordinates $x_{1}, x_{2}$ and $x_{3}$ with three different spatial correlations lengths $L_{1}^{U}, L_{2}^{U}$ and $L_{3}^{U}$.

C. Soize - CMAME - revised version December 2004 


\subsection{Mean model, finite element discretization and static response}

The open bounded domain $\Omega$ of $\mathbb{R}^{3}$ is such that $\left.\Omega=\right] 0,10[\times] 0,1[\times] 0,0.8[$ in meters. This slender domain is fixed on the two sections $x_{1}=0$ and $x_{1}=10$ which means that a Dirichlet condition is applied to the boundary $\Gamma_{0}=\left\{x_{1}=0, x_{2} \in[0,1], x_{3} \in[0,0.8]\right\} \cup\left\{x_{1}=10, x_{2} \in[0,1], x_{3} \in\right.$ $[0,0.8]\}$. The Young modulus and the Poisson coefficient of the homogeneous and isotropic linear elastic material of the mean model are $E=10^{10} \mathrm{~N} / \mathrm{m}^{2}$ and $\nu=0.15$. The matrix $\left[\underline{a}_{n}(\mathbf{x})\right]$ introduced in Section 5.3, belonging to $M_{n}^{+}(\mathbb{R})$ with $n=6$, is independent of $\mathbf{x}$ and is written as

$$
\left[\underline{a}_{n}\right]=\frac{E}{(1+\nu)(1-2 \nu)}\left[\begin{array}{cccccc}
1-\nu & \nu & \nu & 0 & 0 & 0 \\
\nu & 1-\nu & \nu & 0 & 0 & 0 \\
\nu & \nu & 1-\nu & 0 & 0 & 0 \\
0 & 0 & 0 & (1-2 \nu) / 2 & 0 & 0 \\
0 & 0 & 0 & 0 & (1-2 \nu) / 2 & 0 \\
0 & 0 & 0 & 0 & 0 & (1-2 \nu) / 2
\end{array}\right] .
$$

Th elastic domain $\Omega$ is subjected to the static point load $(0,1,0)$ in Newton, applied to the point $(3.5,1,0.4)$ in meter. The finite element model is a regular mesh of $41 \times 3 \times 3=369$ nodes and $40 \times 2 \times 2=160$ finite elements which are 8-nodes solid elements with $2 \times 2 \times 2$ integrating points (see Fig. 5). Therefore there are 1053 degrees of freedom and $N=1280$ integrating points. The neutral fiber is the line defined by $\left\{\mathbf{x}=\left(x_{1}, x_{2}, x_{3}\right), x_{1} \in[0,10], x_{2}=0.5, x_{3}=0.4\right\}$ and is constituted of 41 nodes.

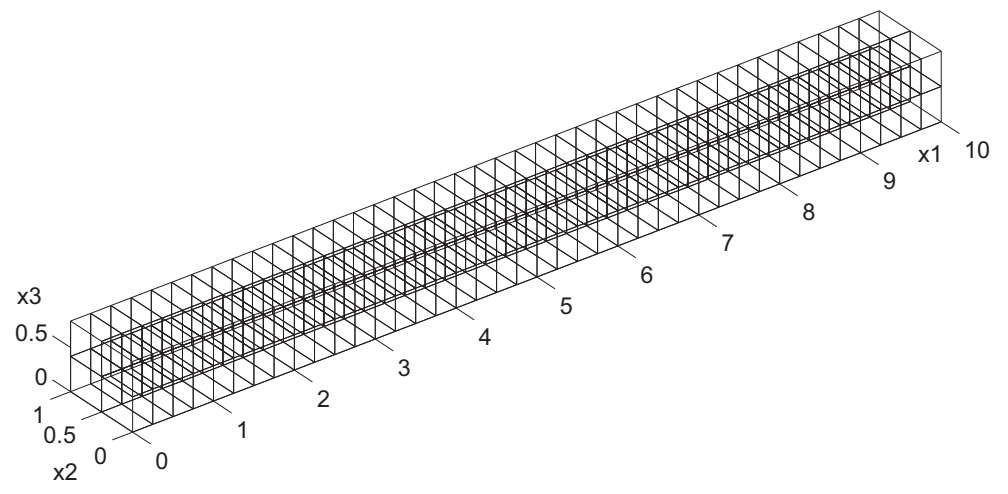

Fig. 5. Finite element model

The displacement field of the mean model is calculated by solving the deterministic linear matrix equation $[\underline{K}] \underline{\mathbf{d}}=\mathbf{f}$ (see Eq. (123)). Figure 6 displays the static deformation of the elastic domain $\Omega$ subjected to the point load. 


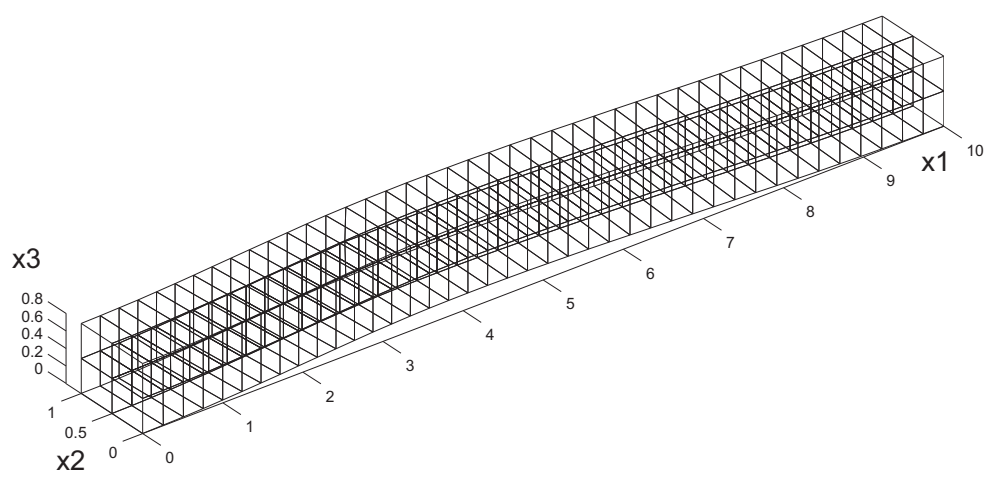

Fig. 6. Static deformation of the three-dimensional domain due to the static point load

\subsection{Stochastic model, computational parameters and stochastic response}

The nonparametric probabilistic model of the elasticity tensor is performed as explained in Section 5.4. The random field $\mathbf{x} \mapsto\left[\mathbf{A}_{n}(\mathbf{x})\right]$, indexed by $\Omega$, with values in $M_{n}^{+}(\mathbb{R})$, with $n=6$, is such that (see Eq. (77)), $\left[\mathbf{A}_{n}(\mathbf{x})\right]=\left[\underline{L}_{n}\right]^{T}\left[\mathbf{G}_{n}(\mathbf{x})\right]\left[\underline{L}_{n}\right]$ in which the matrix $\left[\underline{a}_{n}\right]$, defined by Eq. (155), is written (see Eq. (72)) as $\left[\underline{a}_{n}\right]=\left[\underline{L}_{n}\right]^{T}\left[\underline{L}_{n}\right]$. The stochastic field $\mathbf{x} \mapsto\left[\mathbf{G}_{n}(\mathbf{x})\right]$, indexed by $\mathbb{R}^{3}$, with values in $M_{n}^{+}(\mathbb{R})$, is defined in Section 3.3.2 by Eqs. (29) to (33). The dispersion parameter $\delta_{A_{n}}$ defined by Eq. (87), is $\delta_{A_{n}}=0.1829$. Using Eq. (88), the corresponding parameter $\delta$ defined by Eq. (37), is $\delta=0.2$. The spatial correlation lengths of random field germ $U$, defined by Eq. (13), are such that $L_{1}^{U}=2 m$ and $L_{2}^{U}=L_{3}^{U}=1 m$. The realizations of the random matrices $\left[\mathbf{A}_{n}\left(\mathbf{x}^{1}\right)\right], \ldots,\left[\mathbf{A}_{n}\left(\mathbf{x}^{N}\right)\right]$ at the $N=1280$ integrating points $\mathbf{x}^{1}, \ldots, \mathbf{x}^{N}$ (see Section 6.1 ) are constructed by using the method presented in Section 4.2(vii) and the representation of the random field germ $U$ defined in Section 3.2.3 (ii) (that is to say by Eqs. (19) and (20)). The functions $\eta_{1} \mapsto r_{1}^{A_{n}}\left(\eta_{1}\right)=r^{A_{n}}\left(\eta_{1}, 0,0\right)$, $\eta_{2} \mapsto r_{2}^{A_{n}}\left(\eta_{2}\right)=r^{A_{n}}\left(0, \eta_{2}, 0\right)$ and $\eta_{3} \mapsto r_{3}^{A_{n}}\left(\eta_{3}\right)=r^{A_{n}}\left(0,0, \eta_{3}\right)$ are calculated with Eq. (95) in which the mathematical expectation is estimated by using the Monte Carlo simulation with 20000 realizations. Since $L_{2}^{U}=L_{3}^{U}$, then function $r_{2}^{A_{n}}$ is equal to function $r_{3}^{A_{n}}$. The graphs of functions $\eta_{1} \mapsto r_{1}^{A_{n}}\left(\eta_{1}\right)$ and $\eta_{2} \mapsto r_{2}^{A_{n}}\left(\eta_{2}\right)$ are shown in Fig. 7. For $j=1,2,3$, the spatial correlation lengths $L_{j}^{A_{n}}$ are calculated by Eq. (97) and yields $L_{1}^{A_{n}}=1.97$ and $L_{2}^{A_{n}}=L_{3}^{A_{n}}=0.99$. 

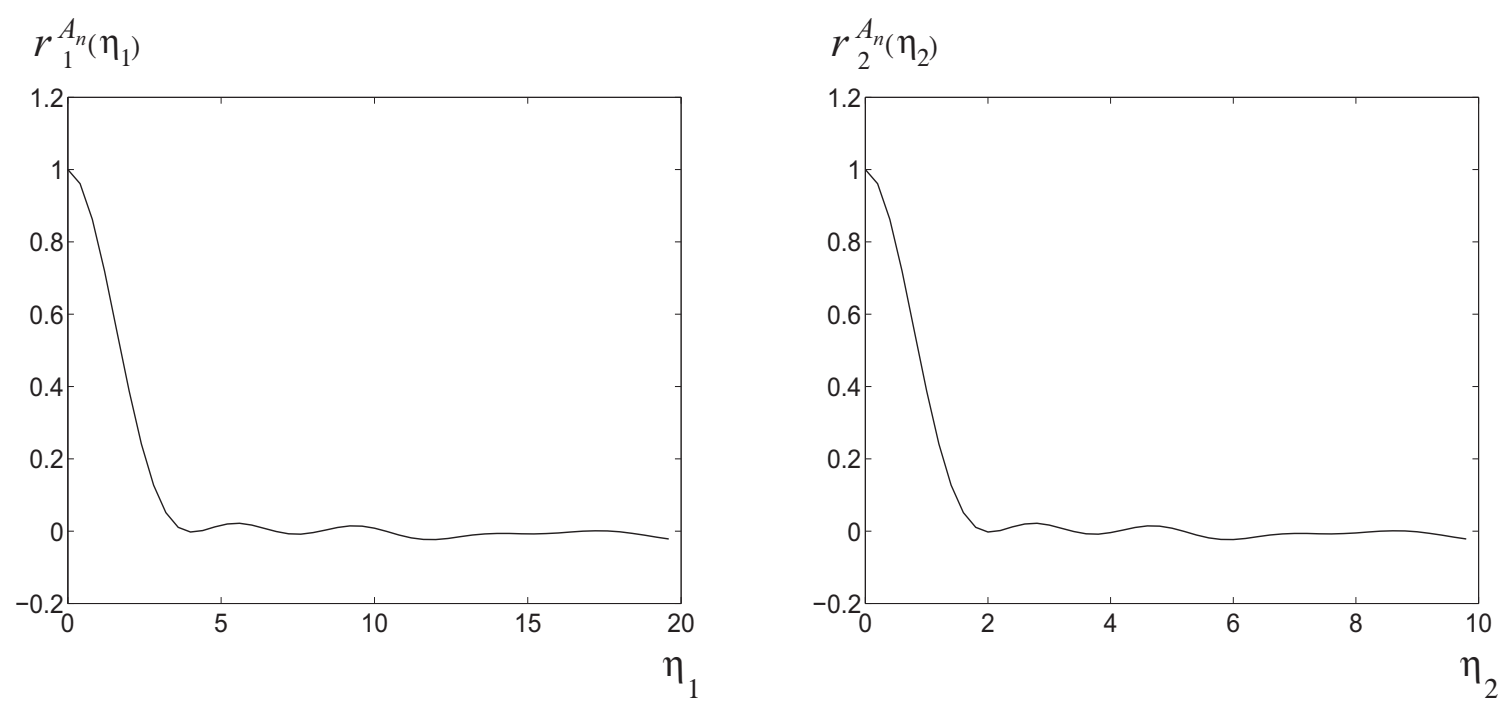

Fig. 7. Graph of the function $\eta_{1} \mapsto r_{1}^{A_{n}}\left(\eta_{1}\right)$ (on the left) and graph of the function $\eta_{2} \mapsto r_{2}^{A_{n}}\left(\eta_{2}\right)$ (on the right).

The random displacement field of the stochastic model is constructed by solving the random linear matrix equation $[\mathbf{K}] \mathbf{D}=\mathbf{f}$ (see Eq. (123)) with the Monte Carlo numerical simulation presented in Section 6.2. Let $n_{s}$ be the number of realization used for the construction of the statistical estimators. Let $\mathbf{D}_{\mathrm{nf}}$ be the $\mathbb{R}^{41}$-valued random vector constituted of the $x_{2}$-DOF for the 41 nodes located in the neutral fiber. The following norm $\left\|\left|\mathbf{D}_{\mathrm{nf}} \|\right|=\left(E\left\{\left\|\mathbf{D}_{\mathrm{nf}}\right\|^{2}\right\}\right)^{1 / 2}\right.$ of the second-order random vector $\mathbf{D}_{\text {nf }}$ can be approximated by $\operatorname{conv}\left(n_{s}\right)$ defined by

$$
\operatorname{conv}\left(n_{s}\right)=\left\{\frac{1}{n_{s}} \sum_{k=1}^{n_{s}}\left\|\mathbf{D}_{\mathrm{nf}}\left(\theta_{k}\right)\right\|^{2}\right\}^{1 / 2}
$$

in which $\theta_{1}, \ldots, \theta_{n_{s}}$ correpond to $n_{s}$ independent realizations in $\Theta$. Mean-square convergence of the random vector $\mathbf{D}_{\mathrm{nf}}$ with respect to the number $n_{s}$ of realization used in the Monte Carlo numerical method, can then be studied by constructing the function $n_{s} \mapsto \operatorname{conv}\left(n_{s}\right)$ which is displayed in Fig. 8. 


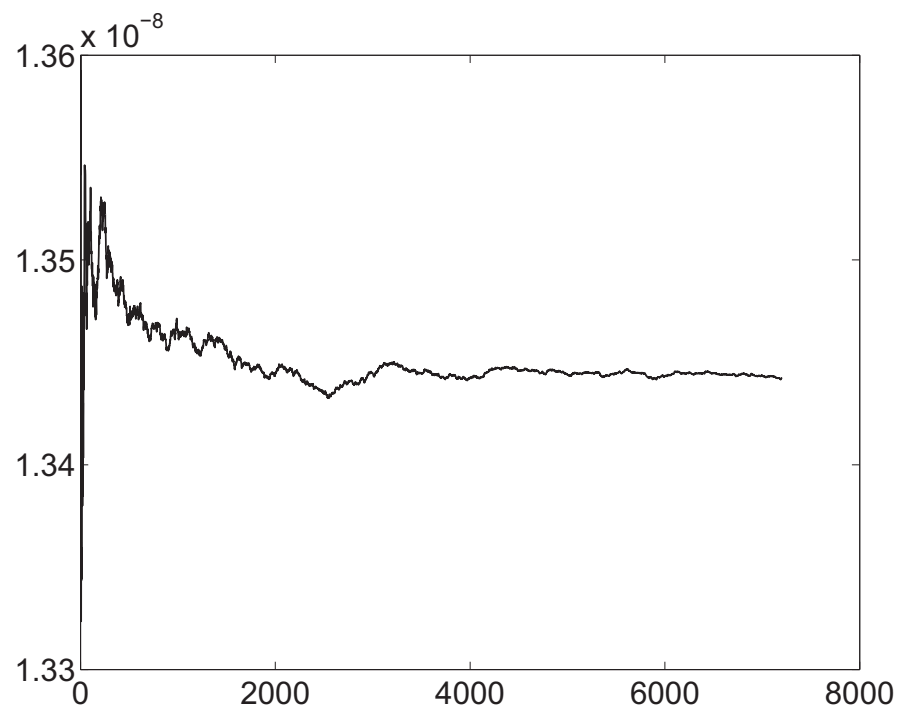

Fig. 8. Mean-square convergence. Graph of function $n_{s} \mapsto \operatorname{conv}\left(n_{s}\right)$

We are interested in the construction of the confidence region associated with a probability level $P_{c}=0.98$ for the random transversal static displacement field $x_{1} \mapsto W\left(x_{1}\right)=U_{2}\left(x_{1}, 0.5,0.4\right)$ of the neutral fiber $\left(x_{1} \in[0,10]\right)$. In fact, this random transversal static displacement field is evaluated at the 41 nodes in the neutral fiber. This confidence region is constructed by using the quantiles. For $x_{1}$ fixed, let $F_{W\left(x_{1}\right)}$ be the cumulative distribution function (continuous from the right) of random variable $W\left(x_{1}\right)$ which is such that $F_{W\left(x_{1}\right)}(w)=P\left(W\left(x_{1}\right) \leq w\right)$. For $0<p<1$, the $p$ th quantile or fractile of $F_{W\left(x_{1}\right)}$ is defined as

$$
\zeta(p)=\inf \left\{w: F_{W\left(x_{1}\right)}(w) \geq p\right\}
$$

Then, the upper envelope $w^{+}\left(x_{1}\right)$ and the lower envelope $w^{-}\left(x_{1}\right)$ of the confidence region are defined by

$$
w^{+}\left(x_{1}\right)=\zeta\left(P_{c}\right) \quad, \quad w^{-}\left(x_{1}\right)=\zeta\left(1-P_{c}\right) \quad .
$$

The estimation of $w^{+}\left(x_{1}\right)$ and $w^{-}\left(x_{1}\right)$ is performed by using the sample quantiles [64]. Let $w_{1}\left(x_{1}\right)=W\left(x_{1} ; \theta_{1}\right), \ldots, w_{n_{s}}\left(x_{1}\right)=W\left(x_{1} ; \theta_{n_{s}}\right)$ be the $n_{s}$ independent realizations of random variable $W\left(x_{1}\right)$ associated with the independent realizations $\mathbf{D}_{\mathrm{nf}}\left(\theta_{1}\right), \ldots, \mathbf{D}_{\mathrm{nf}}\left(\theta_{n_{s}}\right)$. Let $\widetilde{w}_{1}\left(x_{1}\right)<$ $\ldots<\widetilde{w}_{n_{s}}\left(x_{1}\right)$ be the order statistics associated with $w_{1}\left(x_{1}\right), \ldots, w_{n_{s}}\left(x_{1}\right)$. Therefore, one has the following estimation

$$
\begin{aligned}
& w^{+}\left(x_{1}\right) \simeq \widetilde{w}_{j^{+}}\left(x_{1}\right), \quad j^{+}=\operatorname{fix}\left(n_{s} P_{c}\right), \\
& w^{-}\left(x_{1}\right) \simeq \widetilde{w}_{j^{-}}\left(x_{1}\right), \quad j^{-}=\operatorname{fix}\left(n_{s}\left(1-P_{c}\right)\right),
\end{aligned}
$$


in which fix $(z)$ is the integer part of the real number $z$. Figure 9 displays the confidence region of the random transversal static displacement $x_{1} \mapsto W\left(x_{1}\right)$ of the neutral fiber $\left(x_{1} \in[0,10]\right)$, estimated with $n_{s}=7200$.

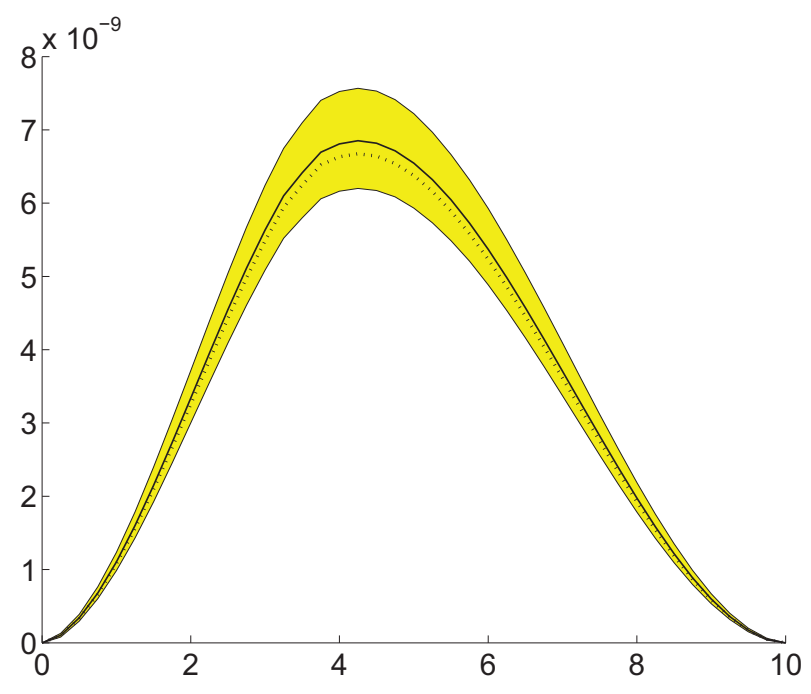

Fig. 9. Confidence region prediction for the random transversal static displacement of the neutral fiber. (1) Graphs of the functions $x_{1} \mapsto\left\{w^{+}\left(x_{1}\right)\right\}$ and $x_{1} \mapsto\left\{w^{-}\left(x_{1}\right)\right\}$ delimiting the confidence region (grey region) of the random transversal static displacement $x_{1} \mapsto W\left(x_{1}\right)$ of the neutral fiber for the stochastic system. (2) Graph of the function $x_{1} \mapsto \underline{u}_{2}\left(x_{1}, 0.5,0.4\right)$ (dotted line) of the transversal static displacement of the neutral fiber for the mean model. (3) Graph of the mean function $x_{1} \mapsto E\left\{W\left(x_{1}\right)\right\}$ (mid solid line) of the random field $x_{1} \mapsto W\left(x_{1}\right)$.

\section{Conclusions}

We have presented the mathematical construction of a class of non Gaussian positive-definite $(n \times n)$ real matrix-valued random fields, indexed by any domain of $\mathbb{R}^{d}$, depending only on a positive-definite matrix-valued mean function and on a smaller number of scalar parameters constituted of a dispersion parameter and $d$ spatial correlation lengths. Such a random field is adapted to the inverse problem relative to the experimental identification. A fundamental mathematical property is proved and allows the ellipticity of stochastic partial differential operators to be obtained. Such a stochastic field can then be used for a nonparametric probabilistic modeling of elliptic partial differential operators. As an example, this non Gaussian positive-definite real matrix-valued random field is used for constructing a nonparametric probabilistic model of the fourth-order elasticity tensor for a random linear elastic material. A numerical example is presented. 


\section{References}

[1] M. Abramowitz, I.A. Stegun, Handbook of Mathematical Functions, Dover Publications, Inc., New York, 1964.

[2] R.J. Adler, An Introduction to Continuity, Extrema, and Related Topics for General Gaussian Processes, Institute of Mathematical Statistics, Lecture Notes - Monograph Series, ISBN 0-94060017-X, 1990.

[3] R.J. Adler, On excursion sets, tube formulas and maxima of random fields, The Annals of Applied Probability, 10(1) (2000) 1-74.

[4] T.W. Anderson, Introduction to Multivariate Statistical Analysis, Wiley, New York, 1958.

[5] J.-M. Azais, C. Delmas, Asymptotic expansions for the distribution of the maximum of Gaussian random fields, Extremes 5(2) (2002) 183-214.

[6] I. Babuska, P. Chatzipantelidis, On solving elliptic stochastic partial differential equations, Computer Methods in Applied Mechanics and Engineering 191 (2002) 4094-4122.

[7] E. Capiez-Lernout, C. Soize, Nonparametric modeling of random uncertainties for dynamic response of mistuned bladed disks, Journal of Engineering for Gas Turbines and Power (accepted for publication in December 2003).

[8] H. Chebli, C. Soize, Experimental validation of a nonparametric probabilistic model of non homogeneous uncertainties for dynamical systems, Journal of the Acoustical Society of America 115(2) (2004) 697-705.

[9] H. Cramer, M.R. Leadbetter, Stationary and Related Stochastic Processes, Wiley, New York, 1967.

[10] R. Dautray, J.-L. Lions, Mathematical Analysis and Numerical Methods for Science and Technology, Springer-Verlag, Berlin, 1992.

[11] B. Debusschere, H.N. Najm, A. Matta, O. Knio, R. Ghanem, o. Le Maître, Protein labeling reactions in electrochemical microchannel flow: numerical simulation and uncertainty propagation, Physics of Fluids, to appear (2004).

[12] C. Delmas, An asymptotic expansion for the distribution of the maximum of a class of Gaussian fields, CRAS, Serie I, Math. 327 (1998) 393-397.

[13] C. Desceliers, C. Soize, S. Cambier, Nonparametric-parametric model for random uncertainties in nonlinear structural dynamics - Application to earthquake engineering, Earthquake Engineering and Structural Dynamics, 33(3) (2004) 315-327. 
[14] O. Ditlevsen, N.J. Tarp-Johansen, Choice of input fields in stochastic finite elements, Probabilistic Engineering Mechanics 14(1-2) (1988) 63-72.

[15] J.L. Doob, Stochastic Processes, John Wiley and Sons, New York, 1953 (Wiley Classics Library Edition Published 1990).

[16] R.M. Dudley, The size of compact subsets of Hilbert spaces and continuity of Gaussian processes, Journal of Functional Analysis 1 (1967) 290-330.

[17] C. Fougeaud, A. Fuchs, Statistique, Dunod, Paris, 1967 ( 2nd ed. 1972).

[18] A.M. Garsia, E. Rodemich, H. Rumsey JR, A real variable lemma and the continuity of paths of some Gaussian processes, Indiana University Mathematics Journal, 20(6) (1970) 565-578.

[19] R. Ghanem, Ingredients for a general purpose stochastic finite elements formulation, Computer Methods in Applied Mechanics and Engineering 168 (1999) 19-34.

[20] R. Ghanem, Stochastic finite elements with multiple random non gaussian properties, Journal of Engineering Mechanics 125 (1) (1999) 26-40.

[21] R. Ghanem, M. Pellissetti, Adaptive data refinement in the spectral stochastic finite element method, Communications in Numerical Methods in Engineering 18 (2) (2002) 141-151.

[22] R. Ghanem, A. Sarkar, Reduced models for the medium-frequency dynamics of stochastic systems, Journal of the Acoustical Society of America 113 (2) (2003) 834-846.

[23] R. Ghanem, S. Dham, Stochastic finite element analysis for multiphase flow in heterogeneous porous media, Transaction Porous Media 32 (1998) 239-262.

[24] R. Ghanem, R.M. Kruger, Numerical solution of spectral stochastic finite element systems, Computer Methods in Applied Mechanics and Engineering 129 (1996) 289-303.

[25] R. Ghanem, J. Red-Horse, Propagation of uncertainty in complex physical systems using a stochastic finite elements approach, Physica D 133 (1999) 137-144.

[26] R. Ghanem, P.D. Spanos, Stochastic Finite Elements: A spectral Approach, revised edition, Dover Publications, New York, 2003.

[27] G.H. Golub, C.F. Van Loan, Matrix Computations, Second Edition, The Johns Hopkins University Press, Baltimore, 1989.

[28] L. Guikhman, A.V. Skorokhod, The Theory of Stochastic Processes, Springer-Verlag, Berlin, 1979.

[29] R.Z. Hasminskii, Stochastic Stability of Differential Equations, Sijthoff and Noordhoff, The Netherlands, 1980.

[30] E.J. Haug, K.K. Choi,V. Komkov, Design Sensitivity Analysis of Structural Systems, Academic 
Press, London, SanDiego, 1986.

[31] R.A. Ibrahim, Parametric Random Vibration, John Wiley and Sons, New York, 1985.

[32] N. Ikeda, S. Watanabe, Stochastic Differential Equations and Diffusion Processes, North-Holland, Amsterdam, 1981.

[33] K. Itô, H.P. McKean, Diffusion Processes and Their Sample Paths, Springer-Verlag, Berlin, 1965.

[34] E.T. Jaynes, Information theory and statistical mechanics, Physical Review 106 (4) (1957) 620-630 $\& 108$ (2) (1957) 171-190.

[35] G.M. Jenkins, D.G. Watt, Spectral Analysis and its Applications, Holden Day, San Francisco, 1968.

[36] G. Jumarie, Maximum Entropy, Information without Probability and Complex Fractals, Kluwer Academic Publishers, Dordrecht, 2000.

[37] J.N. Kapur, H.K. Kesavan, Entropy Optimization Principles with Applications, Academic Press, San Diego, 1992.

[38] M.G. Kendall, A. Stuart, The Advanced Theory of Statistics, Griffin, London, 1966.

[39] M. Kleiber, D.H. Tran, T.D. Hien, The Stochastic Finite Element Method, John Wiley and Sons, New York, 1992.

[40] P.E. Kloeden, E. Platen, Numerical Solution of Stochastic Differential Equations, Springer-Verlag, Berlin Heidelberg, 1992.

[41] P. Krée, C. Soize, Mathematics of Random Phenomena, Reidel Publishing Company, Dordrecht, Holland, 1986.

[42] M.R. Leadbetter, G. Lindgren, H. Rootzen, Extremes and Related Properties of Random Sequences and Processes, Springer-Verlag, New York, 1983.

[43] O. Le Maître, O. Knio, H.N. Najm, R. Ghanem, A stochastic projection method for fluid flow. I. Basic formulation, Journal of Computational Physics 173 (2001) 481-511.

[44] O. Le Maître, M.T. Reagan, H.N. Najm, R. Ghanem, O. Knio, A stochastic projection method for fluid flow. II. Random process, Journal of Computational Physics 173 (2001) 481-511.

[45] Y.K. Lin, Probabilistic Theory of Structural Dynamics, McGraw-Hill, New York, 1967 (Reprint R.E. Krieger, Melbourne, Fla., 1976)

[46] W.K. Liu, T. Belytschko, A. Mani, Random field finite elements, International Journal of Numerical Methods in Engineering 23 (1986) 1832-1845.

[47] P.K. MacKeown, Stochastic Simulation in Physics, Springer-Verlag, Singapore, 1997.

[48] M.L. Mehta, Random Matrices, Revised and Enlarged Second Edition, Academic Press, New York, 
1991.

[49] J.T. Oden, J.N. Reddy, Variational Methods in Theoretical Mechanics, Springer Verlag, 2nd edition, 1983 (1st edition, 1976).

[50] R. Ohayon, C. Soize, Structural Acoustics and Vibration, Academic Press, London, San Diego, 1998.

[51] B. Oksendal, Stochastic Differential Equations, Sixth Edition, Springer-Verlag, Berlin, 2003.

[52] A.V. Oppenheim, R.N. Schaffer, Digital Signal Processing, Prentice Hall, Englewood Cliffs, New Jersey, 1975.

[53] V.I. Piterbarg, Asymptotic Methods in the Theory of Gaussian Processes and Fields, Translations of Mathematical Monographs, Volume 148, American Mathematical Society, Providence, Rhode Island, 1996.

[54] F. Poirion, C. Soize, Numerical methods and mathematical aspects for simulation of homogeneous and non homogeneous Gaussian vector fields, pp. 17-53 in Probabilistic Methods in Applied Physics, P. Krée and W. Wedig (Eds.), Lecture Notes in Physics, ISBN 3-540-60214-3, SpringerVerlag, Berlin, Heidelberg, 1995

[55] M.B. Priestley, Spectral Analysis and Time Series, Academic Press, New York, 1981.

[56] M.B. Priestley, Nonlinear and Non-Stationary Time Series Analysis, Academic Press, New York, 1988.

[57] P. Protter, Stochastic Integration and Differential Equations - A New Approach, Springer-Verlag, Berlin, 1990.

[58] M. Reed, B. Simon, Methods of Modern Mathematical Physics, Academic Press, New York, 1980.

[59] J.B. Roberts, P.D. Spanos, Random Vibration and Statistical Linearization, Wiley, New York, 1990.

[60] Y.A. Rozanov, Random Fields and Stochastic Partial Differential Equations, Kluwer Academic Publishers, Dordrecgt, The Netherlands, 1998.

[61] R.Y. Rubinstein, Simulation and the Monte Carlo Method, John Wiley and Sons, New York, 1981.

[62] G.I. Schueller (Ed.), A state-of-the-art report on computational stochastic mechanics, Probabilistic Engineering Mechanics 12(4) (1997) 197-313.

[63] G.I. Schueller and M. Shinozuka (Eds.), Stochastic Methods in Structural Dynamics, Martinus Nijhoff Publication, Dordrecht, The Netherlands, 1987.

[64] R.J. Serfling, Approximation Theorems of Mathematical Statistics, John Wiley \& Sons, 1980.

[65] C.E. Shannon, A mathematical theory of communication, Bell System Technology Journal 27 (1948)

C. Soize - CMAME - revised version December 2004 
$379-423 \& 623-659$.

[66] M. Shinozuka, Simulations of multivariate and multidimensional random processes, J. Acoust. Soc. Amer. 39(1)(1971) 357-367.

[67] M. Shinozuka, C.M. Jan, Digital simulation of random processes and its applications, Journal of Sound and Vibration 25 (1972) 111-128.

[68] M. Shinozuka, Y.K. Wen, Monte Carlo solution of nonlinear vibration, AIAA Journal 10(1) (1972) $37-40$.

[69] M. Shinozuka, G. Deodatis, Response variability of stochastic finite element systems, Journal of Engineering Mechanics, 114(3) (1988) 499-519.

[70] A.V. Skorohod, Random Linear Operators, Reidel Publishing Company, Dordrecht, , Holland, 1984.

[71] K. Sobczyk, Stochastic Differential Equations with Applications to Physics and Engineering, Kluewer, Dordrecht, Boston, 1991.

[72] C. Soize, Mathematical Methods in Signal Analysis (in French), Masson, Paris, 1993.

[73] C. Soize, The Fokker-Planck Equations for Stochastic Dynamical Systems and its Explicit Steady State Solutions, World Scientific, Singapore, 1994.

[74] C. Soize, A nonparametric model of random uncertainties for reduced matrix models in structural dynamics, Probabilistic Engineering Mechanics 15(3) (2000) 277-294.

[75] C. Soize, Maximum entropy approach for modeling random uncertainties in transient elastodynamics, Journal of the Acoustical Society of America 109 (5) (2001) 1979-1996.

[76] C. Soize, Transient responses of dynamical systems with random uncertainties, Probabilistic Engineering Mechanics 6 (4) (2001), 363-372.

[77] C. Soize, Nonlinear dynamical systems with nonparametric model of random uncertainties, Uncertainties in Engineering Mechanics Journal 1(1) (2001) 1-38.

[78] C. Soize, Random matrix theory and non-parametric model of random uncertainties, Journal of Sound and Vibration 263 (2003) 893-916.

[79] C. Soize, Uncertain dynamical systems in the medium-frequency range, Journal of Engineering Mechanics 129 (9) (2003) 1017-1027.

[80] C. Soize, H. Chebli, Random uncertainties model in dynamic substructuring using a nonparametric probabilistic model, Journal of Engineering Mechanics, ASCE 129 (4)(2003) 449-457.

[81] C. Soize, Random matrix theory for modeling uncertainties in computational mechanics, Computer Methods in Applied Mechanics and Engineering 194(12-16)(2005) 1333-1366 .

C. Soize - CMAME - revised version December 2004 
[82] C. Soize and R. Ghanem, Physical systems with random uncertainties : Chaos representation with arbitrary probability measure, SIAM Journal On Scientific Computing 26(2)(2004) 395-410.

[83] T.T. Soong, Random Diffential Equations in Science and Engineering, Acadelic Press, New York, 1973.

[84] P.D. Spanos, B. A. Zeldin, Galerkin sampling method for stochastic mechanics problems, Journal of Engineering Mechanics 120(5) (1994) 1091-1106.

[85] P.D. Spanos, R.G. Ghanem, Stochastic finite element expansion for random media, ASCE Journal of Engineering Mechanics 115(5) (1989) 1035-1053.

[86] D.W. Stroock, S.R.S. Varadhan, Multidimensional Diffusion Processes, Springer-Verlag, Berlin Heidelberg, 1979.

[87] G.S. Székely, G.I. Schuëller, Computational procedure for a fast calculation of eigenvectors and eigenvalues of structures with random properties, Computer Methods in Applied Mechanics and Engineering 191 (2001) 799-816.

[88] F. Treves, Basic Linear Partial Differential Equations, Academic Press, New York, 1975.

[89] E. Vanmarcke, M. Grigoriu, Stochastic finite element analysis of simple beams, Journal of Engineering Mechanics, ASCE 109 (5) (1983) 1203-1214.

[90] M. Wschebor, Surfaces Aléatoires, Lecture Notes in Mathematics, 1147, Springer-Verlag, Berlin Heidelberg, 1980.

[91] O. C. Zienkiewicz, R. L. Taylor, The Finite Element Method, Fifth edition, Vol. 1 to 3, ButterworthHeinemann, Oxford, 2000. 